\title{
THE RADIAL VELOCITY EXPERIMENT (RAVE): SECOND DATA RELEASE
}

T. Zwitter ${ }^{1}$, A. Siebert 2,3 , U. Munari ${ }^{4}$, K. C. Freeman ${ }^{5}$, A. Siviero ${ }^{4}$, F. G. Watson ${ }^{6}$, J. P. Fulbright ${ }^{7}$, R. F. G. Wyse ${ }^{7}$, R. Campbell ${ }^{2,8}$, G. M. Seabroke ${ }^{9}, 10$, M. Williams ${ }^{2,5}$, M. Steinmetz ${ }^{2}$, O. Bienaymé ${ }^{3}$, G. Gilmore ${ }^{9}$, E. K. Grebel ${ }^{11}$, A. Helmi ${ }^{12}$, J. F. Navarro ${ }^{13}$, B. Anguiano ${ }^{2}$, C. Boeche ${ }^{2}$, D. Burton ${ }^{6}$, P. Cass $^{6}$, J. Dawe ${ }^{6,23}$, K. Fiegert ${ }^{6}$, M. Hartley ${ }^{6}$, K. Russell ${ }^{6}$, L. Veltz ${ }^{2,3}$, J. Bailin ${ }^{14}$, J. Binney ${ }^{15}$, J. Bland-Hawthorn ${ }^{16}$, A. Brown ${ }^{17}$, W. Dehnen ${ }^{18}$, N. W. Evans ${ }^{9}$, P. Re Fiorentin ${ }^{1}$, M. Fiorucci ${ }^{4}$, O. Gerhard ${ }^{19}$, B. Gibson ${ }^{20}$, A. Kelz ${ }^{2}$, K. KuJKen ${ }^{12}$, G. MatiJevič ${ }^{1}$, I. Minchev ${ }^{21}$, Q. A. Parker ${ }^{8}$, J. Peñarrubia ${ }^{13}$, A. Quillen ${ }^{21}$, M. A. Read ${ }^{22}$, W. Reid ${ }^{8}$, S. Roeser ${ }^{11}$, G. Ruchti ${ }^{7}$, R.-D. Scholz ${ }^{2}$,

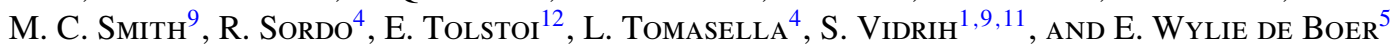

${ }^{1}$ University of Ljubljana, Faculty of Mathematics and Physics, Ljubljana, Slovenia

${ }^{2}$ Astrophysikalisches Institut Potsdam, Potsdam, Germany

${ }^{3}$ Observatoire de Strasbourg, Strasbourg, France

${ }^{4}$ INAF, Osservatorio Astronomico di Padova, Sede di Asiago, Italy

${ }^{5}$ RSAA, Australian National University, Canberra, Australia

${ }^{6}$ Anglo Australian Observatory, Sydney, Australia

${ }^{7}$ Johns Hopkins University, Baltimore, MD, USA

${ }^{8}$ Macquarie University, Sydney, Australia

${ }^{9}$ Institute of Astronomy, University of Cambridge, UK

${ }^{10} \mathrm{e} 2 \mathrm{v}$ Centre for Electronic Imaging, School of Engineering and Design, Brunel University, Uxbridge, UK

${ }^{11}$ Astronomisches Rechen-Institut, Center for Astronomy of the University of Heidelberg, Heidelberg, Germany

${ }^{12}$ Kapteyn Astronomical Institute, University of Groningen, Groningen, The Netherlands

${ }^{13}$ University of Victoria, Victoria, Canada

${ }^{14}$ Centre for Astrophysics and Supercomputing, Swinburne University of Technology, Hawthorn, Australia

${ }^{15}$ Rudolf Pierls Center for Theoretical Physics, University of Oxford, UK

${ }^{16}$ Institute of Astronomy, School of Physics, University of Sydney, NSW 2006, Australia

${ }^{17}$ Sterrewacht Leiden, University of Leiden, Leiden, The Netherlands

${ }^{18}$ University of Leicester, Leicester, UK

${ }^{19}$ MPI für extraterrestrische Physik, Garching, Germany

${ }^{20}$ University of Central Lancashire, Preston, UK

${ }^{21}$ University of Rochester, Rochester NY, USA

${ }^{22}$ University of Edinburgh, Edinburgh, UK

Received 2008 January 8; accepted 2008 April 15; published 2008 June 13

\begin{abstract}
We present the second data release of the Radial Velocity Experiment (RAVE), an ambitious spectroscopic survey to measure radial velocities and stellar atmosphere parameters (temperature, metallicity, surface gravity, and rotational velocity) of up to one million stars using the $6 \mathrm{dF}$ multi-object spectrograph on the $1.2 \mathrm{~m}$ UK Schmidt Telescope of the Anglo-Australian Observatory (AAO). The RAVE program started in 2003, obtaining medium resolution spectra (median $R=7500$ ) in the Ca-triplet region (8410-8795 $\AA$ ) for southern hemisphere stars drawn from the Tycho- 2 and SuperCOSMOS catalogues, in the magnitude range $9<I<12$. Following the first data release, the current release doubles the sample of published radial velocities, now containing 51,829 radial velocities for 49,327 individual stars observed on 141 nights between 2003 April 11 and 2005 March 31. Comparison with external data sets shows that the new data collected since 2004 April 3 show a standard deviation of $1.3 \mathrm{~km} \mathrm{~s}^{-1}$, about twice as good as for the first data release. For the first time, this data release contains values of stellar parameters from 22,407 spectra of 21,121 individual stars. They were derived by a penalized $\chi^{2}$ method using an extensive grid of synthetic spectra calculated from the latest version of Kurucz stellar atmosphere models. From comparison with external data sets, our conservative estimates of errors of the stellar parameters for a spectrum with an average signal-to-noise ratio $(\mathrm{S} / \mathrm{N})$ of $\sim 40$ are $400 \mathrm{~K}$ in temperature, 0.5 dex in gravity, and 0.2 dex in metallicity. We note however that, for all three stellar parameters, the internal errors estimated from repeat RAVE observations of 855 stars are at least a factor 2 smaller. We demonstrate that the results show no systematic offsets if compared to values derived from photometry or complementary spectroscopic analyses. The data release includes proper motions from Starnet2, Tycho-2, and UCAC2 catalogs and photometric measurements from Tycho-2 USNO-B, DENIS, and 2MASS. The data release can be accessed via the RAVE Web site: http://www.rave-survey.org and through CDS.
\end{abstract}

Key words: catalogs - stars: fundamental parameters - surveys

Online-only material: color figures

\section{INTRODUCTION}

This paper presents the second data release from the Radial Velocity Experiment (RAVE), an ambitious spectroscopic sur-

\footnotetext{
${ }^{23}$ Deceased.
}

vey of the southern sky which has already observed over 200,000 stars away from the plane of the Milky Way $\left(|b|>25^{\circ}\right)$ and with apparent magnitudes $9<I_{\text {DENIS }}<13$. The paper follows the first data release, described in Steinmetz et al. (2006), hereafter Paper I. It doubles the number of published radial velocities. For the first time it also uses spectroscopic analysis to provide 
information on values of stellar parameters: temperature, gravity, and metallicity. Note that the last in general differs from iron abundance, because metallicity is the proportion of matter made up of all chemical elements other than hydrogen and helium in the stellar atmosphere. Stellar parameters are given for the majority of the newly published stars. This information is supplemented by additional data from the literature: stellar position, proper motion, and photometric measurements from DENIS, Two Micron All Sky Survey (2MASS), and Tycho surveys.

Scientific uses of such a data set were described in Steinmetz (2003). They include the identification and study of the current structure of the Galaxy and of remnants of its formation, recent accretion events, as well as discovery of individual peculiar objects and spectroscopic binary stars. Kinematic information derived from the RAVE dataset has been used (Smith et al. 2007) to constrain the Galactic escape speed at the solar radius to $v_{\mathrm{esc}}=536_{-44}^{+58} \mathrm{~km} \mathrm{~s}^{-1}(90 \%$ confidence $)$. The fact that $v_{\mathrm{esc}}^{2}$ is significantly greater than $2 v_{\text {circ }}^{2}$ (where $v_{\text {circ }}=220 \mathrm{~km} \mathrm{~s}^{-1}$ is the local circular velocity) is a model-independent confirmation that there must be a significant amount of mass exterior to the solar circle, i.e., it convincingly demonstrates the presence of a dark halo in the Galaxy. A model-dependent estimate yields the virial mass of the Galaxy of $1.31_{-0.49}^{+0.97} \times 10^{12} M_{\odot}$ and the virial radius of $297_{-44}^{+60} \mathrm{kpc}$ ( $90 \%$ confidence). Veltz et al. (2008) discussed kinematics toward the Galactic poles and identified discontinuities that separate a thin disk, thick disk, and a hotter component. Seabroke et al. (2008) searched for infalling stellar streams on to the local Milky Way disk and found that it is devoid of any vertically coherent streams containing hundreds of stars. The passage of the disrupting Sagittarius dwarf galaxy leading tidal stream through the solar neighborhood is therefore ruled out. Additional ongoing studies have been listed in Paper I.

The structure of this paper is as follows. Section 2 is a description of the observations, which is followed by a section on data reduction and processing. Data quality is discussed in Section 4, with a particular emphasis on a comparison of the derived values of stellar parameters with results from an analysis of external data sets. Section 5 is a presentation of the data product, followed by concluding remarks on the results in the context of current large spectroscopic surveys.

\section{OBSERVATIONS}

RAVE is a magnitude-limited spectroscopic survey. For this reason it avoids any kinematic bias in the target selection. The wavelength range 8410-8795 $\AA$ overlaps with the photometric Cousins $I$ band. However, the DENIS and 2MASS catalogs were not yet available at the time of planning of the observations we present here. So this data release uses the same input catalog as Paper I: the bright stars were selected using $I$ magnitudes estimated from the Tycho- $2 V_{\mathrm{T}}$ and $B_{\mathrm{T}}$ magnitudes (Høg et al. 2000) and the faint ones were chosen by their I magnitudes in the SuperCOSMOS Sky Survey (Hambly et al. 2001), hereafter SSS. Transformations to derive the $I$ magnitude and its relation to the DENIS I magnitude values are discussed in Paper I. There we also comment on the fact that SuperCOSMOS photographic I magnitudes show an offset with respect to DENIS I magnitudes (Figure 1). So, although the initial magnitude limit of the survey was planned to be 12.0, the actual limit is up to 1 mag fainter.

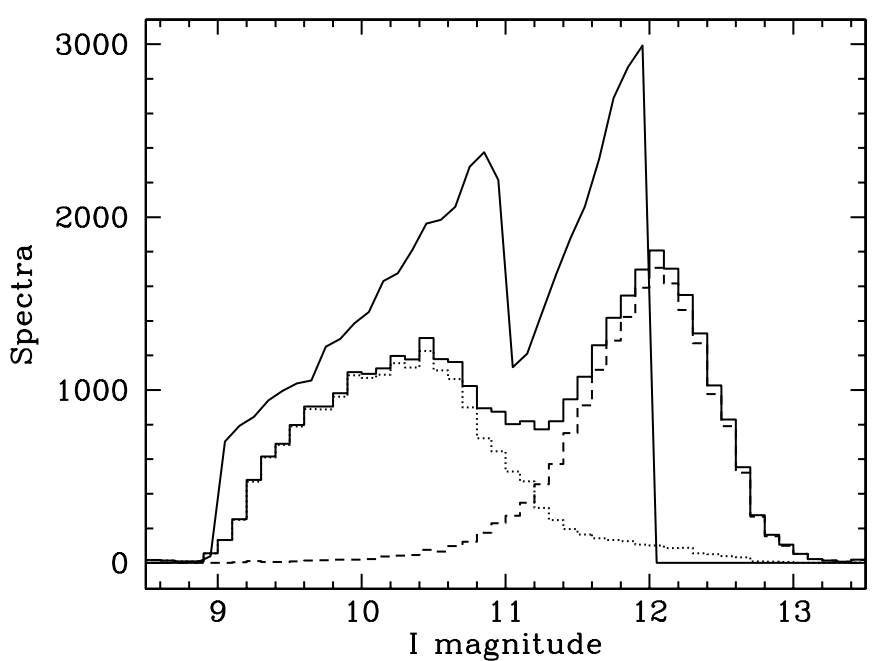

Figure 1. Cousins $I$-band magnitudes of RAVE spectra in the second data release. The smooth line denotes magnitudes derived from Tycho-2 and SSS survey photometry which were used as an input catalog for RAVE. The solid line histogram depicts DENIS I magnitudes for the $77 \%$ of stars which are also in the second release of the DENIS catalog. Short- and long-dashed lines are histograms of DENIS I magnitudes for stars from the Tycho-2 and SSS surveys, respectively. Test fields close to the Galactic plane $\left(|b|<25^{\circ}\right)$ are not plotted.

The survey spans a limited range in apparent magnitude; still it probes both the nearby and the more distant galaxy. Typical distances for $\mathrm{K} 0$ dwarfs are between 50 and $250 \mathrm{pc}$, while the $\mathrm{K} 0$ giants are located at distances of $0.7-3 \mathrm{kpc}$.

The instrumental setup is similar to the one used in Paper I. Two field plates with robotically positioned fibers are used in turn in the focus of the UK Schmidt Telescope at the AngloAustralian Observatory (AAO). A field plate covers a 5.7 ${ }^{\circ}$ field of view and feeds light to up to 150 fibers, each with an angular diameter of $6.7^{\prime \prime}$ on the sky. One should be careful to avoid chance superpositions with target stars when using such wide fibers. As a precaution we avoid regions close to the Galactic plane $\left(|b|<25^{\circ}\right)$ or dense stellar clusters. Also, all candidate stars are visually checked for possible contamination prior to observing using the 1 arcmin SSS thumbnails from the on-line SSS $R$-band data.

Each field plate contains 150 science fibers, with additional bundles used for guiding. A robot positioner configures the plate for each field by moving each fiber end to the desired position. The associated mechanical stress occasionally causes the fiber to break, so it needs to be repaired. A typical fiber is broken after every two years of use on average, and is repaired in the next eight months. Figure 2 shows the number of fibers which were used successfully to collect star light for each of the 517 pointings. The number varies with time. A period of decline is followed by a sharp rise after the repair of broken fibers on the corresponding field plate. Each pointing was typically used to successfully observe 106 stars. An additional nine or ten fibers were used to monitor the sky background.

The light is dispersed by a bench-mounted Schmidt-type spectrograph to produce spectra with a resolving power of $R \sim 7500$. The main improvement introduced since the first data release is the use of a blue light blocking filter (Schott OG531), which blocks the second-order spectrum. This allows for an unambiguous placement of the continuum level and so permits the derivation of values of stellar parameters, in addition to the radial velocity (RV). The introduction of the blocking filter lowers the number of collected photons by only $\sim 25 \%$, so we 


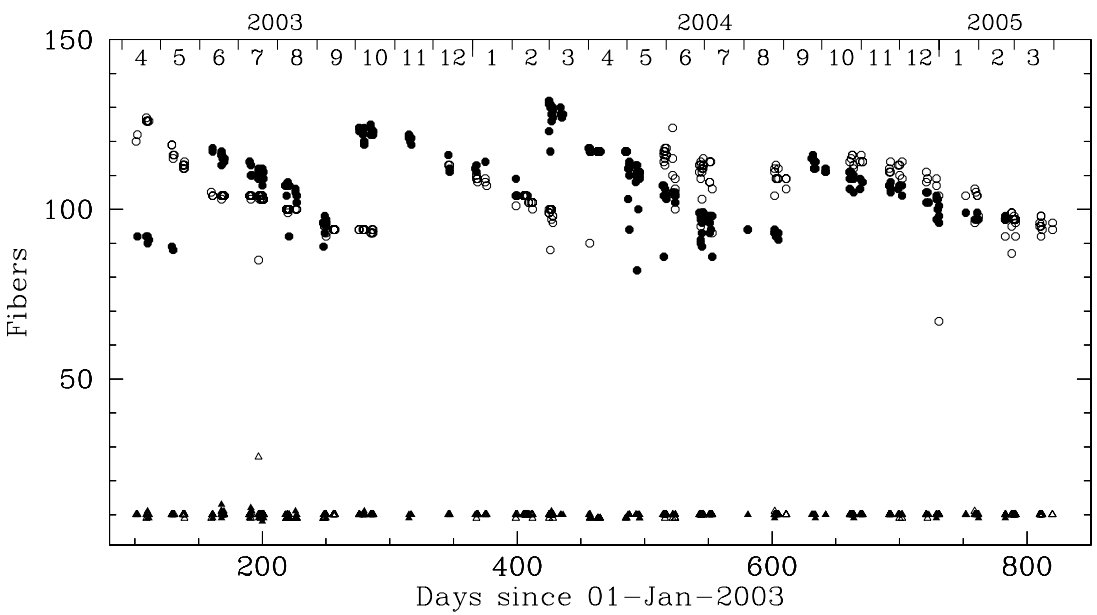

Figure 2. Number of fibers observing stars (circles) and sky background (triangles) for fields in the second data release. Filled symbols mark observations obtained with fiber plate 1 and open symbols those with plate 2. Test fields close to the Galactic plane $\left(|b|<25^{\circ}\right)$ have been omitted from the graph.

Table 1

Observing Statistics

\begin{tabular}{lcc}
\hline \hline & $\begin{array}{c}\text { All } \\
\text { data }\end{array}$ & $\begin{array}{c}\text { New data } \\
\text { in this DR }\end{array}$ \\
\hline Number of nights of observation & 141 & 72 \\
Number of fields (incl. repeats) & 517 & 266 \\
Sky area covered $\left(\mathrm{deg}^{2}\right)$ & 7200 & 2440 \\
Stellar spectra & 51,829 & 25,850 \\
Number of different stars & 49,327 & 24,010 \\
Number of stars observed once & 47,492 & 22,676 \\
Number of stars observed twice & 1618 & 1232 \\
Number of stars observed 3 times & 124 & 25 \\
Number of stars observed 4 times & 2 & 1 \\
Number of stars observed 5 times & 2 & 0 \\
Number of stars observed 6 times & 0 & 0 \\
Number of stars observed 7 times & 1 & 1 \\
Number of stars observed 8 times & 88 & 75 \\
\hline
\end{tabular}

Note. The middle column counts all data in the present data release and the right one only data obtained after 2004 April 3 , i.e., new to this data release.

decided to keep the same observing routine as described in Paper I. The observation of a given field consists of five consecutive $10 \mathrm{~min}$ exposures, which are accompanied by flatfield and neon arc calibration frames.

Note that we use two field plates on an alternating basis (fibers from one fiber plate are being configured while we observe with the other field plate). So fibers from a given field plate are mounted to the spectrograph slit prior to the observation of each field. To do this the cover of the spectrograph needs to be removed, so its temperature may change abruptly. The associated thermal stress implies that it is best to use the flatfield and neon arc lamp exposures obtained immediately after the set of scientific exposures when the spectrograph is largely thermally stabilized. For all data new to this data release, we ensured that such flat-field and arc lamp exposures were obtained and used in the data reduction.

Observations were obtained between 2003 April 11 and 2005 March 31. The observations obtained since 2004 April 3 yielded data which were not published in Paper I, so they are new to this data release. Statistics on the number of useful nights, of field centers, and of stellar spectra are given in Table 1. These numbers make the present, second data release about twice as large as that presented in Paper I. Stars were mostly observed only once, but 75 stars from the field centered on R.A. $=16^{\mathrm{h}} 07^{\mathrm{m}}$, decl. $=-49^{\circ}$ were deliberately observed eight times to study their variability.

Observations are limited to the southern hemisphere and have a distance of at least $25^{\circ}$ from the Galactic plane (except for a few test fields). Their distribution is plotted in Figure 21. The unvisited area is concentrated around the Galactic plane and in the direction of the Magellanic Clouds.

\section{DATA REDUCTION AND PROCESSING}

The data reduction is performed in several steps, as follows.

1. Quality control of the acquired data.

2. Spectra reduction.

3. RV determination and estimation of physical stellar parameters.

In the first step, the RAVEdr software package and plotting tools are used to make a preliminary estimate of data quality in terms of signal levels, focus quality, and possible interference patterns. This serves two goals: to quickly determine which observations need to be repeated because of unsatisfactory data quality and to exclude any problematic data from further reduction steps. For the first data release $17 \%$ of all pointings were classified as problematic, while in this data release the overall dropout rate fell to $13 \%$. Problematic data are kept separately and are not part of this data release. The next two steps of the data reduction process are described below.

\subsection{Spectra Reduction}

We use a custom set of IRAF routines which have been described in detail in Paper I. Here we highlight only the improvements introduced for reduction of data new to this data release.

The use of the blue light blocking filter permits a more accurate flat-fielding of the data. The spectra have a length of 1031 pixels, and are found to cover a wavelength interval of $384.6 \pm 1.7 \AA$. The resolving power is the same as estimated in Paper I; we use the value of $R \simeq 7500$ throughout. The camera of the spectrograph has a very fast focal ratio $(F / 1)$. The associated optical aberrations at large off-axis angles imply that the central wavelength of the spectrograph is not constant, but depends on the fiber number (Figure 3). This means that 


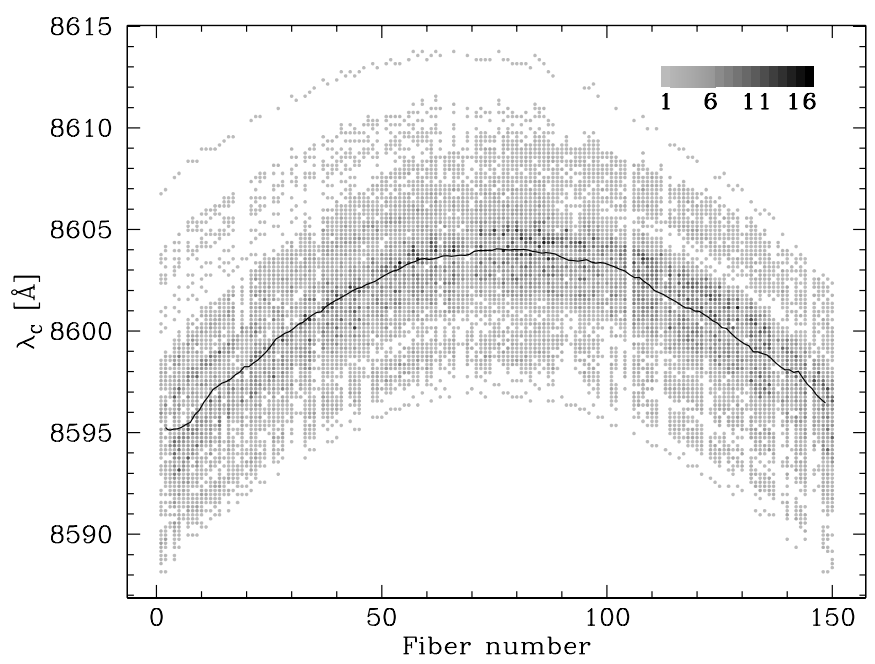

Figure 3. Variation of central wavelength as a function of the fiber number for data new to this release. Shades of gray code the number of spectra in a certain bin, as given in the key. The line follows half-point central wavelengths as a function of the fiber number.

the wavelengths covered by a spectrum depend on its fiber number. Also, any residual cross-talk between the spectra in adjacent fibers is generally shifted in wavelength. This makes an iterative procedure to remove illumination from adjacent fibers even more important (see Paper I for details). The peak of central wavelengths around the half-point of their distribution shows that our instrumental setup remained quite stable for one year when the data new to this data release were obtained.

The determination of RV and stellar parameters is based on the 788 pixels of the central part of the wavelength range only (8449.77 $\AA<\lambda<8746.84 \AA$ ). This avoids telluric absorption lines and a ghost image caused by internal reflections of nondispersed light at the borders of the wavelength range which are occasionally present and could jeopardize the results, as described in Paper I. The edges of the spectral interval are also avoided because of a poorer focus, lower resolving power, and a lower quality of the wavelength calibration.

Figure 4 plots the average ADU count level of the central part of the final 1D spectrum, and per $1 \mathrm{~h}$ of exposure time, as a function of DENIS I magnitude. Only data new to this data release are plotted. The line follows the relation

$$
N_{\text {counts }}=10^{-0.4\left(I_{\text {DENIS }}-20.25\right)},
$$

where the constant term is the mode of the magnitude-corrected count distribution. These count levels are 0.25 mag below those in Paper I. The difference is due to the second-order blocking filter. Note however that the filter allowed for a more accurate flat-fielding, and so better determined count levels. This information has been used in data quality control.

\section{2. $R V$ Determination}

The general routine stayed the same as described in detail in Paper I. Radial velocities are computed from sky-subtracted normalized spectra, while sky-unsubtracted spectra are used to compute the zero-point correction. The latter is needed because of thermal variations of the spectrograph which cause a shift of the order of one-tenth of a pixel or $1.5 \mathrm{~km} \mathrm{~s}^{-1}$. Radial velocities are computed from cross-correlation with an extensive library of synthetic spectra. A set of 57,943 spectra degraded to the resolving power of RAVE from Munari et al. (2005a) is used. It is

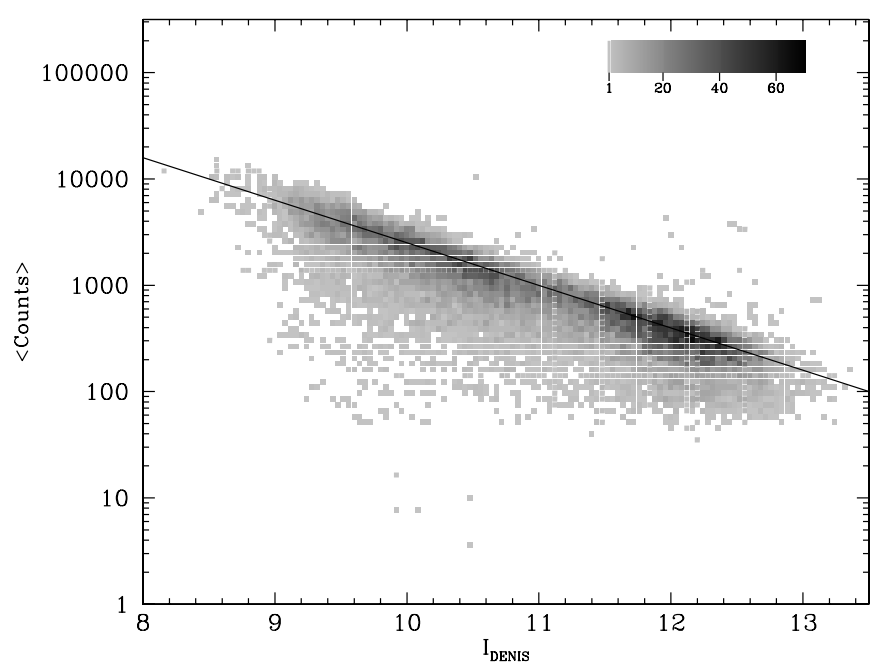

Figure 4. Average number of counts per pixel per hour of exposure time as a function of DENIS I magnitude. Shades of gray code the number of spectra in a certain bin, as given in the key. The average count level is calculated from the central part of the spectrum only ( $8449.77 \AA \leqslant \lambda \leqslant 8746.84 \AA)$. The inclined line follows Equation (1).

based on the latest generation of Kurucz models. It covers all loci of non-degenerate stars in the $H-R$ diagram, with metallicities in the range of $-2.5 \leqslant[\mathrm{M} / \mathrm{H}] \leqslant+0.5$. Most spectra have a microturbulent velocity of $2 \mathrm{~km} \mathrm{~s}^{-1}$ (with additional entries for 1 and $4 \mathrm{~km} \mathrm{~s}^{-1}$ ), while the $\alpha$-enhancements of $[\alpha / \mathrm{Fe}]=0.0$ and +0.4 are used. The use of the blue blocking filter simplifies the computations, as no contribution from the second-order spectrum needs to be considered. Both the observed spectra and theoretical templates are normalized prior to the radial velocity measurement. We use IRAF's task continuum with a two-piece cubic spline. The rejection criteria used in ten consecutive iterations of the continuum level are asymmetric ( $1.5 \sigma$ low and $3 \sigma$ high).

Kurucz synthetic spectra used in cross-correlation do not include corrections of RV due to convective motions in the stellar atmosphere or due to a gravitational redshift of light leaving the star (F. Castelli 2007, private communication). The combined shift is in the range of $-0.4 \mathrm{~km} \mathrm{~s}^{-1}$ for $F$ dwarfs to $+0.4 \mathrm{~km} \mathrm{~s}^{-1}$ for $K$ dwarfs (Gullberg \& Lindegren 2002), while the near absence of gravitational redshift in giants causes a $\sim 0.4 \mathrm{~km} \mathrm{~s}^{-1}$ shift between giants and dwarfs. The exact value of these corrections is difficult to calculate, so we follow the Resolution C1 of the IAU General Assembly in Manchester (Rickman 2001) and report the heliocentric radial velocities without corrections for gravitational or convective shifts in the stellar atmosphere. Note however that these values may be different from the line-of-sight component of the velocity of the stellar center of mass (Lindegren 1999; Latham 2001).

In the final data product we report the heliocentric RV and its error, together with the value of the applied zero-point velocity correction, the RV of sky lines, and their correlation properties. A detailed description of the data release is given in Section 5.

\subsection{Stellar Parameter Determination}

The name of the survey suggests that RAVE is predominantly a RV survey. However, the spectral type of the survey stars is generally not known and the input catalog does not use any color criterion, so RAVE stars are expected to include all evolutionary stages and a wide range of masses in the $H-R$ diagram. The properties of the stellar spectra in the wavelength interval used 
by RAVE strongly depend on the values of the stellar parameters (Munari et al. 2001). While the Ca II IR triplet is almost always present, the occurrence and strength of Paschen, metallic, and molecular lines depends on temperature, gravity, and metallicity (see, e.g., Figure 4 in Zwitter et al. 2004). So we cannot adopt the common practice of using a small number of spectral templates to derive the RV alone, as has been commonly done at, e.g., the ELODIE spectrograph at Observatoire de Haute Provence $(\mathrm{OHP})$. We therefore construct the best matching template from a large library of synthetic Kurucz spectra (see Section 3.2). The parameters of the best matching spectrum are assumed to present the true physical parameters in the stellar atmosphere.

Two comments are in order before we outline the template spectrum construction method. First, the template library only covers normal stars. So peculiar objects cannot be classified correctly. Such objects include double-lined spectroscopic binaries and emission line objects. Sometimes a peculiar nature of the spectrum can be inferred from a poor match of the templates, despite a high $\mathrm{S} / \mathrm{N}$ of the observed spectrum.

The second important point concerns the non-orthogonality of the physical parameters we use. This is demonstrated in Figure 5: the wavelength ranges with flux levels sensitive to a change in temperature overlap with those sensitive to metallicity and the rotational velocity. On the other hand, sensitivity to changes in both gravity and temperature depend on spectral type and class. The intermittent lines in Figure 5 mark wavelengths where the normalized flux level changes for at least $3 \%$ if the value of one of the parameters is modified by a given amount (temperature by $500 \mathrm{~K}$, gravity or metallicity by $0.5 \mathrm{dex}$, or rotational velocity by $30 \mathrm{~km} \mathrm{~s}^{-1}$ ). We note that a $3 \%$ change is marginally detectable in a typical RAVE spectrum with $\mathrm{S} / \mathrm{N}=40$, but the non-orthogonality of individual parameters can present a serious problem (see also Figure 1 in Zwitter 2002). If the temperature or gravity were known a priori, the ambiguities would be largely resolved. An obvious idea is to use photometric colors to constrain the value of stellar temperature. Unfortunately, the errors of current photometric surveys are too large: a change of 0.03 mag in $J-K$ corresponds to a shift of $230 \mathrm{~K}$ in temperature in a mid- $G$ main-sequence (MS) star. Also, stellar colors may be seriously compromised by interstellar extinction or by stellar binarity. We therefore decided not to use any outside information but to base our estimates of stellar parameters exclusively on spectral matching. This may change in the future when results of multicolor and multi-epoch all-sky photometric surveys such as SkyMapper (Keller et al. 2007) will become available.

Our parameter estimation procedure makes use of a full set of theoretical templates. They span a grid in six parameters: temperature, gravity, metallicity, $\alpha$-enhancement, microturbulent, and rotational velocity. The sampling in gravity, metallicity, and temperature is very good, with $\gtrsim 9$ tabulated values for the former two and even more for the temperature. On the other hand, the current synthetic library contains only one non-solar $\alpha$-enhancement value $([\alpha / \mathrm{Fe}]=+0.4)$ and only up to three values of microturbulent velocity $\left(1,2,4 \mathrm{~km} \mathrm{~s}^{-1}\right.$, but only $2 \mathrm{~km} \mathrm{~s}^{-1}$ is available for the whole grid). So we decided to publish values of temperature, gravity, and metallicity. The $\alpha$-enhancement values are also listed but they should be interpreted with caution, as they are derived from two grid values only. These two values may not span the whole range of $\alpha$-enhancement which is present in nature. Also, the error of $\alpha$-enhancement can be comparable to the whole range of the grid in this parameter (see Section 3.3.5). Microturbulent velocity values are not published, because their errors are typically much larger than the range of microturbulent velocities in the grid. Similarly, the rather low resolving power of RAVE spectra does not allow the determination of rotational velocities $\left(V_{\text {rot }}\right)$ for slow rotators which represent the vast majority of RAVE stars. Hence the rotational velocity is not published, but fast rotators will be discussed in a separate paper. So we aim at the estimation of three stellar parameters: effective temperature $\left(T_{\text {eff }}\right)$, gravity $(\log g)$, and metallicity $([\mathrm{M} / \mathrm{H}])$. The adopted reference system of these parameters is the latest set of Kurucz template spectra. Next we describe the inverse method used to derive values of stellar parameters.

\subsubsection{Method}

To derive the stellar parameters, we use a penalized $\chi^{2}$ technique to construct a synthetic spectrum matching the observed spectrum (for other uses of similar methods, see, e.g., Pichon et al. 2002; Ocvirk et al. 2006a). The observed spectrum is modeled as a weighted sum of template spectra with known parameters and it is assumed that the stellar parameters follow the same weight relation. The continuous problem is therefore written as

$$
\left\{\begin{array}{l}
\mathcal{F}_{\boldsymbol{P}^{\prime}}(\lambda)=\int \tilde{w}(\boldsymbol{P}) \mathcal{S}(\lambda, \boldsymbol{P}) \mathrm{d}^{6} \boldsymbol{P} \\
\boldsymbol{P}^{\prime}=\int \tilde{w}(\boldsymbol{P}) \boldsymbol{P} \mathrm{d}^{6} \boldsymbol{P},
\end{array}\right.
$$

where $\mathcal{F}$ is the spectrum we want the stellar parameters for, $\mathcal{S}(\lambda, \boldsymbol{P})$ are the template spectra with known stellar parameters $\boldsymbol{P}=\left(T_{\text {eff }}, \log g,[\mathrm{M} / \mathrm{H}],[\alpha / \mathrm{Fe}], V_{\text {rot }}, \mu\right)^{\top}, \boldsymbol{P}^{\prime}$ is the stellar parameter set we want to measure and $\tilde{w}(\boldsymbol{P})$ is the weight function we try to recover. In the perfect case, where we have an infinite number of template spectra and the observed spectrum depends only on the stellar parameters (perfect match between the observed and model spectra $), \tilde{w}(\boldsymbol{P})=\delta\left(\boldsymbol{P}-\boldsymbol{P}^{\prime}\right)$. In a real case where noise plays an important role and a real spectrum cannot be perfectly reproduced, $\tilde{w}(\boldsymbol{P})$ is not a Dirac function but a smooth function which is non-zero on a limited range. Also, we have the additional constraint $\int \tilde{w}(\boldsymbol{P}) \mathrm{d}^{6} \boldsymbol{P}=1$.

In the more general case, we have access to a limited number of templates and the problem becomes discrete. The problem can then be rewritten as

$$
\left\{\begin{array}{l}
\mathcal{S}_{\boldsymbol{P}}(\lambda)=\sum_{i} w_{i} \cdot \mathcal{S}_{\boldsymbol{P}_{i}}(\lambda) \\
\boldsymbol{P}=\sum_{i} w_{i} \cdot \boldsymbol{P}_{i},
\end{array}\right.
$$

where $w_{i}$ is the discrete form of $\tilde{w}(\boldsymbol{P})$.

This problem is ill-conditioned, the number of template spectra being larger than the number of pixels, and the information contained in a spectrum being largely redundant. Therefore, we make use of penalization terms to regularize the solution. Also, the recovered weights must be positive to have a physical meaning, which changes the problem from linear to nonlinear. The following paragraphs will briefly present the linear problem which has a well-defined solution before entering the realm of the nonlinear problem. For a full discussion and description of the method, the reader is referred to Pichon et al. (2002), Ocvirk et al. (2006a, 2006b), and references therein.

\subsubsection{Linear Inverse Problem}

The discrete problem of Equation (3) can be written in a matrix form. Calling $\tilde{\boldsymbol{y}}=\left(\mathcal{F}\left(\lambda_{1}\right), \ldots, \mathcal{F}\left(\lambda_{n}\right)\right)^{\top}$ the observed 


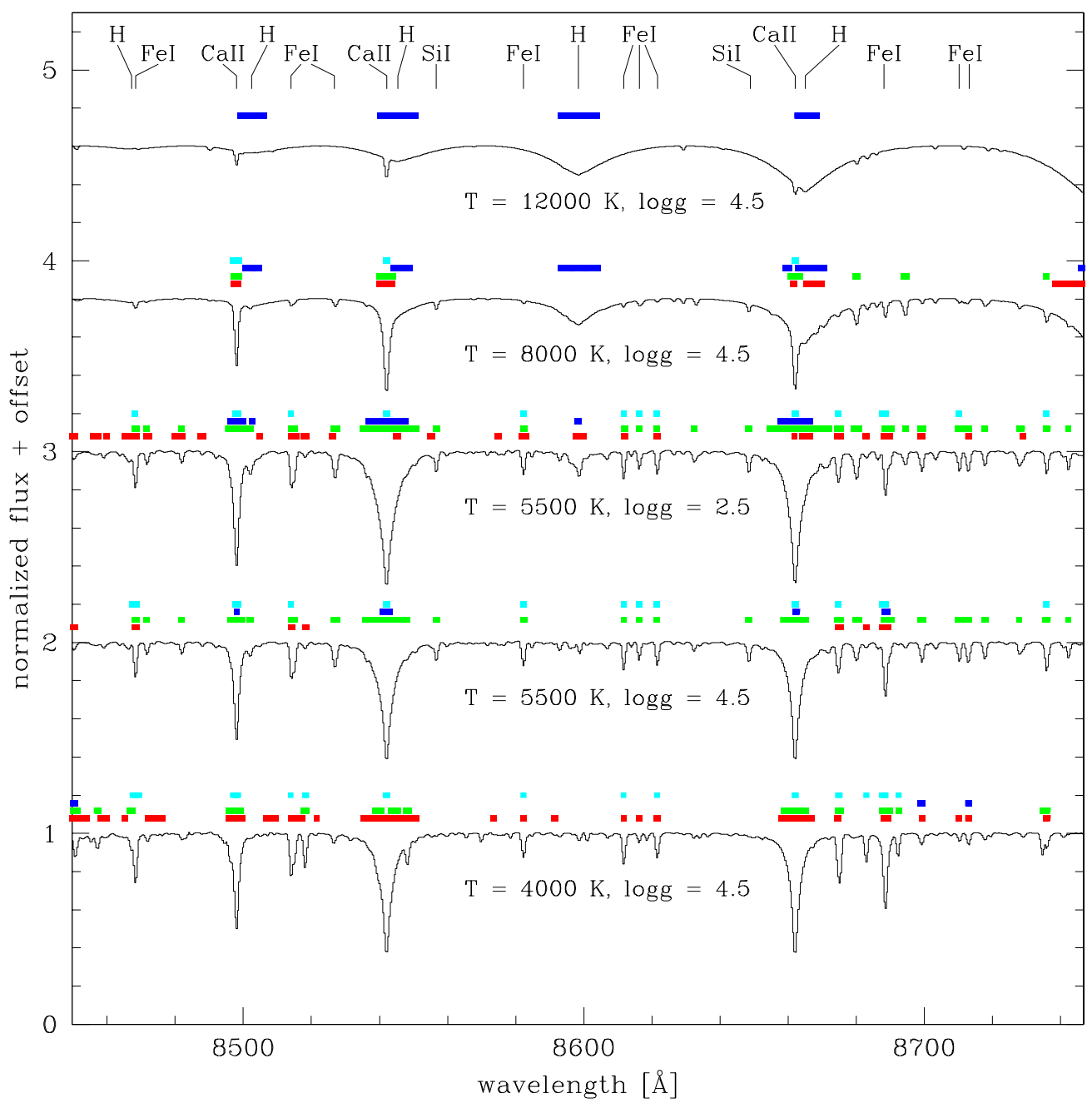

Figure 5. Sensitivity of synthetic spectra to stellar parameters. These are synthetic spectra of non-rotating stars with solar metallicity and microturbulent velocity of $2 \mathrm{~km} \mathrm{~s}^{-1}$. Intermittent lines mark regions where a change in one of the parameters causes a change of at least $3 \%$ in normalized flux. From bottom up the intermittent lines mark flux changes for: a $500 \mathrm{~K}$ decrease in temperature (red), a 0.5 dex decrease in metallicity (green), a 0.5 dex decrease in gravity (blue), a $30 \mathrm{~km} \mathrm{~s}^{-1}$ increase in rotational velocity (cyan). The wavelength range of the spectra is the one actually used for the determination of stellar parameters.

spectrum, $\boldsymbol{x}=\left(w_{1}, \ldots, w_{m}\right)^{\top}$ the array of weights, $\boldsymbol{a}=$ $\left(\mathcal{S}_{i=1}, \ldots, \mathcal{S}_{i=m}\right)^{\top}$ the library of template spectra, and $\boldsymbol{b}=$ $\left(\boldsymbol{P}_{i=1}, \ldots, \boldsymbol{P}_{i=m}\right)^{\top}$ the array of parameters, the problem then reads

$$
\left\{\begin{array}{l}
\tilde{y}=a \cdot x+e \\
P=b \cdot x
\end{array}\right.
$$

where $\boldsymbol{e}$ accounts for the noise in the observed spectrum. $\boldsymbol{a}$ is also referred to as the model matrix or kernel.

Using Bayes' theorem, solving Equation (3) or (4) is equivalent to maximizing the a posteriori conditional probability density $f_{\text {post }}(\boldsymbol{x} \mid \tilde{\boldsymbol{y}})$ defined as

$$
f_{\text {post }}(\boldsymbol{x} \mid \tilde{\boldsymbol{y}})=\mathcal{L}(\tilde{\boldsymbol{y}} \mid \boldsymbol{x}) f_{\text {prior }}(\boldsymbol{x}) .
$$

Here, $f_{\text {prior }}(\boldsymbol{x})$ is our prior on the stellar parameters and $\mathcal{L}(\tilde{\boldsymbol{y}} \mid \boldsymbol{x})$ is the likelihood of the data given the model.

In the case of Gaussian errors, the likelihood is

$$
\mathcal{L}(\tilde{\boldsymbol{y}} \mid \boldsymbol{x}) \propto \exp \left(-\frac{1}{2}(\tilde{\boldsymbol{y}}-\boldsymbol{a} \cdot \boldsymbol{x})^{\top} \cdot \boldsymbol{W} \cdot(\tilde{\boldsymbol{y}}-\boldsymbol{a} \cdot \boldsymbol{x})\right),
$$

where the expression in the exponent is the $\chi^{2}$ operator:

$$
\chi^{2}(\tilde{y} \mid x)=(\tilde{y}-\boldsymbol{a} \cdot \boldsymbol{x})^{\top} \cdot \boldsymbol{W} \cdot(\tilde{y}-\boldsymbol{a} \cdot \boldsymbol{x}),
$$

$\boldsymbol{W}$ is the inverse of the covariance matrix of the noise; $\boldsymbol{W}=$ $\operatorname{cov}(\boldsymbol{e})^{-1}$. Maximizing $f_{\text {post }}(\boldsymbol{x} \mid \tilde{\boldsymbol{y}})$ is equivalent to minimizing the penalty operator $\mathcal{Q}(\boldsymbol{x})$ given by

$$
\begin{aligned}
\mathcal{Q}(\boldsymbol{x}) & =\chi^{2}(\tilde{\boldsymbol{y}} \mid \boldsymbol{x})-2 \log \left(f_{\text {prior }}(\boldsymbol{x})\right) \\
& =\chi^{2}(\tilde{\boldsymbol{y}} \mid \boldsymbol{x})+\lambda \boldsymbol{R}(\boldsymbol{x}),
\end{aligned}
$$

where in the second form, the a priori probability density has been rewritten as a penalization or regularization operator $\boldsymbol{R}$ and $\lambda$ is a Lagrange multiplier.

When $\boldsymbol{R}(\boldsymbol{x})$ is a quadratic function, e.g. $\boldsymbol{R}(\boldsymbol{x})=\boldsymbol{x}^{\top} \cdot \boldsymbol{K} \cdot \boldsymbol{x}$ and $\boldsymbol{K}=\boldsymbol{L}^{\top} \cdot \boldsymbol{L}$, the problem has a well-defined solution:

$$
\boldsymbol{x}=\left(\boldsymbol{a}^{\top} \cdot \boldsymbol{W} \cdot \boldsymbol{a}+\lambda \boldsymbol{K}\right)^{-1} \cdot \boldsymbol{a}^{\top} \cdot \boldsymbol{W} \cdot \tilde{\boldsymbol{y}},
$$

and the optimal $\lambda$ is given by the generalized cross-validation $(\mathrm{GCV}): \lambda_{0}=\operatorname{GCV}(\lambda)=\min _{\lambda}\left\{\frac{\|(\mathbf{1}-\tilde{a}) \tilde{y}\|^{2}}{[\operatorname{trace}(\mathbf{1}-\tilde{a})]^{2}}\right\}$, where $\tilde{\boldsymbol{a}}=$ $\boldsymbol{a} \cdot\left(\boldsymbol{a}^{\top} \cdot \boldsymbol{W} \cdot \boldsymbol{a}+\lambda \boldsymbol{K}\right)^{-1} \cdot \boldsymbol{a}^{\top} \cdot \boldsymbol{W}$.

Using Equation (10), $\boldsymbol{x} \in \mathbb{R}^{m}$ and the weights $\boldsymbol{x}_{i}$ can have negative values. Negative weights have no physical meaning and will result in nonphysical solutions. We therefore require that $\boldsymbol{x} \in \mathbb{R}^{+m}$, which leads to the nonlinear problem discussed below. 


\subsubsection{Nonlinear Extension}

Unfortunately, there is no simple extension from the analytic linear problem to the nonlinear case, and there is no analytic solution for the minimum of $\mathcal{Q}$. In the nonlinear regime, the minimum of $\mathcal{Q}$ must be obtained using efficient minimization algorithms and can be computer intensive.

Nevertheless, as stressed by Ocvirk et al. (2006a), solving the nonlinear case also has advantages. First, we will obtain a physically motivated solution (with positive or null weights everywhere); then imposing positivity reduces significantly the allowed parameter space and reduces the level of the Gibbs phenomenon (or ringing artifacts) in the solution. This comes at the price of a higher computing time and asymmetric (nonGaussian) errors.

To ensure that the weights are positive, we pose $x=\exp (\alpha)$ and solve Equation (3) for $\alpha$. The exponential transform has the property that while $\alpha \in \mathbb{R}^{m}, \boldsymbol{x} \in \mathbb{R}^{+* m}$ which ensures that the weights $\boldsymbol{x}$ are strictly positive. Equation (9) can be rewritten as

$$
\begin{aligned}
\mathcal{Q}(\alpha)= & (\tilde{y}-\boldsymbol{a} \cdot \exp \alpha)^{\top} \cdot \boldsymbol{W} \cdot(\tilde{y}-\boldsymbol{a} \cdot \exp \alpha) \\
& +\lambda_{1} \boldsymbol{P}_{1}(\alpha)+\lambda_{2} \boldsymbol{P}_{2}(\alpha)+\cdots,
\end{aligned}
$$

and the problem now is to find the minimum of $\mathcal{Q}(\alpha)$ for $\alpha$. Note that in the last equation, the regularization operator $\boldsymbol{R}$ has been split in a set of regularization operators, each with its own Lagrange parameter. The penalization operators will be discussed in the next section.

We mentioned that in the linear case, the GCV provides an optimal value for the Lagrange parameter; however, in the nonlinear case, this definition is no longer valid. Also, no method is known that allows a quick estimate of the optimal $\lambda$ for the nonlinear problem. In our case, we estimate the proper Lagrange parameter values by means of numerical simulations using synthetic spectra and Gaussian noise. The $\lambda$ used in the pipeline were chosen to optimize the computation time and the accuracy (highest possible accuracy in a minimal computation time). It must be stressed here that the Lagrange parameters, obtained from numerical simulations, may not be optimal as the simulations cannot cover all the parameter space and as the idealized simulations do not incorporate all the ingredients of a real spectrum. Nevertheless, the simulations allow us to find a solution for the Lagrange parameters matching predefined requirements.

Finally, using the exponential transform can cause the solution to be unbound. For example, we expect the weights of spectra far away from the true solution to be zero. In this case, for $\boldsymbol{x}_{i}=0, \alpha_{i} \rightarrow-\infty$ and the solution is unbound. This problem can be solved using an additional term in the regularization, penalizing solutions where $\alpha_{i}$ becomes lower than a predefined threshold. For example, in the case of continuum-subtracted spectra, the threshold can be set from $\boldsymbol{x}_{i}=\frac{10^{-3}}{N_{\mathrm{lib}}}$ to $\frac{10^{-5}}{N_{\mathrm{lib}}}, N_{\mathrm{lib}}$ being the number of spectra in the library, ensuring that the contribution of a template spectrum away from the solution is negligible.

\subsubsection{Penalization}

The problem of determining the stellar parameters from a RAVE spectrum is ill-conditioned and requires regularization in order to recover a physically meaningful solution. Also, the size of the synthetic spectra library we are using is too large to enable us to process a RAVE spectrum within a realistic time frame considering the number of spectra to process.
Our first operation reduces the size of the parameter space by selecting templates according to a $\chi^{2}$ criterion. We use the transform

$$
\exp \left(\alpha_{i}^{\prime}\right)=\exp \left(\alpha_{i}\right) \theta\left(\boldsymbol{P}_{i}\right)
$$

and solve Equation (11) replacing $\alpha$ by $\alpha^{\prime} . \theta\left(\boldsymbol{P}_{i}\right)$ is a gate function in the $6 \mathrm{D}$ stellar parameter space. In the $1 \mathrm{D}$ case, it reads

$$
\theta\left(\boldsymbol{P}_{i}\right)=\left\{\begin{array}{l}
1 \text { if }-\frac{1}{2}<i-i^{\prime} \leqslant \frac{1}{2} \text { and } \chi^{2}\left(\boldsymbol{P}_{i}^{\prime}\right)<\chi_{\lim }^{2} \\
0 \text { otherwise. }
\end{array}\right.
$$

At each point on the grid defined by the library, the derivative $\theta(\boldsymbol{P})$ is 0 . Therefore, solving Equation (11) for $\alpha^{\prime}$ is equivalent to solving the same equation for $\alpha$ but on a reduced subset of $\alpha$ matching the $\chi^{2}$ condition, and we shall drop the prime in the following.

We choose to use a $\chi^{2}$ criterion to select the subset in order to include local minima with a $\chi^{2}$ value close to the minimum $\chi^{2}$. This selection criterion avoids potential problems where the noise, ghost, or cosmic rays create spurious minima which could lead to biases in the estimated stellar parameters. Care must be taken when selecting the $\chi^{2}$ limit as, if the number of spectra in the subset of templates is not large enough, biases can be introduced in the solution. The limit $\chi_{\lim }^{2}$ was chosen according to numerical simulation using the synthetic template library. Simulations have shown that, using Equation (11), at least the 150 template spectra from the lowest $\chi^{2}$ must be used to minimize the reconstruction errors and avoid biases. As those simulations were run using idealized spectra, in practice $\chi_{\lim }^{2}$ is set to the 300th lowest $\chi^{2}$ for a given spectrum. This leads to a subsample of the library containing between 2 and 4 values per parameter, depending on the location in the parameter space. This number is lower than $3^{6}$ which would be the number of spectra used for a quadratic interpolation on a complete $6 \mathrm{D}$ grid, and is due to the fact that the stellar parameter space is not evenly covered by the library. The average number of direct neighbors (on a grid point next to a given parameter) is 85 , varying between 1 and 314 .

Reducing the number of template spectra does not solve the ill-conditioned nature of the problem, even if the number of templates becomes lower than the number of pixels. This is due to the fact that the pixel values are not independent and the information on effective temperature, gravity, etc. is redundant in a spectrum. To regularize the problem we use the property that, in the idealized continuous case, the solution $w(\boldsymbol{P})$ is expected to be close to a Gaussian function centered on the true solution. Therefore, we expect the discrete solution to follow the same behavior and we require the solution $\boldsymbol{x}$ to be smooth in the parameter space. Nevertheless, as in the real case the solution might have local minima because of the noise or features in the spectrum, we do not impose any particular shape for the solution and we keep the method non-parametric. ${ }^{24}$ We only require that the variation of the weights in the parameter space be locally smooth. We define the penalization operator $P_{2}$ as

$$
P_{2}(\alpha)=\exp (\alpha)^{\top} \cdot \boldsymbol{L}^{\top} \cdot \boldsymbol{L} \cdot \exp (\alpha),
$$

where

$$
\boldsymbol{L}_{i, j} \propto\left\{\begin{array}{l}
\frac{-1}{\langle d\rangle_{\mathcal{N i}}} \quad i \neq j, i \in \mathcal{N}_{i} \\
1 i=j \\
0 \text { otherwise. }
\end{array}\right.
$$

\footnotetext{
${ }^{24}$ The method is non-parametric in the sense that no functional form is
} imposed for the array of parameters. 
$d$ is the distance in the parameter space defined as

$$
d\left(\boldsymbol{P}_{i}, \boldsymbol{P}_{j}\right)=\sqrt{\sum_{k} \frac{\left(P_{i, k}-P_{j, k}\right)^{2}}{\sigma_{k}^{2}}},
$$

$k$ being an index over the dimensions of the stellar parameter space, $\langle d\rangle_{\mathcal{N} i}$ the mean distance over a fixed neighborhood $\mathcal{N}$ of the point defined by the index $i$ in the parameter space, and $\sigma_{k}$ the dispersion in the stellar parameter $k$. In practice, the neighborhood is set to the 40 closest points in $\boldsymbol{P}$ which is approximately half the average number of neighbors. The fact that not the entire neighborhood is used to compute the average distance does not introduce errors as the operator is local and all the templates will contribute as the operator is applied over the entire set of templates. Note here that $\langle d\rangle_{\mathcal{N} i}$ is always lower than 1 . With this definition, $\boldsymbol{L}^{\top} \cdot \boldsymbol{L}$ will be large for $i=j$, negative in the surrounding of $i$ in the parameter space and 0 outside. $P_{2}$ is then large when a large value for a given template $i$ is not balanced by its neighborhood, penalizing strong local variations like peaks of width lower than $\sigma_{k}$ of the library.

To derive the stellar parameters, the method presented above is applied to continuum-subtracted spectra and to recover the proper continuum level we have the additional constraint that $\sum_{i} \exp \left(\alpha_{i}\right)=1$. Therefore, we add a third penalization term to ensure that the sum of the weights $\sum \exp (\alpha)$ is 1 . For clean spectra, this last penalization can be omitted. But in the case of RAVE, a ghost can affect the blue part of some spectra and there may be residuals of cosmic ray strikes. So imposing the continuum level enables us to avoid potential problems in automatic processing. The operator $P_{3}$ is defined as

$$
P_{3}(\alpha)=1-\exp \left(-\frac{\left(1-\sum \exp (\alpha)\right)^{2}}{2 \sigma_{3}^{2}}\right)
$$

This operator has an inverted Gaussian behavior around $\sum \exp (\alpha)=1$, with $P_{3}=0$ for $\sum \exp (\alpha)=1$ and $P_{3}=1$ away from this value. To estimate the stellar parameters, we use $\sigma_{3}=0.01$ or $1 \%$ of the continuum value.

\subsubsection{Validation of the Method}

To establish the validity of our approach to recover the stellar parameters in the RAVE regime, we tested the algorithm on a series of 20,000 synthetic spectra built using the same template library. As the accuracy of the method depends on the resolution and wavelength interval (the Lagrange parameters must be defined separately for each instrument and library), we do not try to validate the method outside of our observational regime and this section will focus on an idealized case mimicking the RAVE spectra. A complete discussion of error estimates and zero point offsets, comparing our measurements to other sources, is presented in Section 4.2.2.

The synthetic spectra are randomly generated from a linear interpolation of the library and three ingredients are added:

1. a Gaussian white noise with a signal-to-noise ratio $(\mathrm{S} / \mathrm{N})$ in the range [10-40];

2. an RV mismatch up to $5 \mathrm{~km} \mathrm{~s}^{-1}$;

3. continuum structures amounting to up to $5 \%$ of the continuum level.

These three ingredients were added to mimic observed and expected features in the RAVE spectra: a random noise level typical of the RAVE spectra, a mean internal RV error of $\sim 5 \mathrm{~km} \mathrm{~s}^{-1}$ after the first RV estimation ${ }^{25}$, and residual continuum features that can be left after data reduction. The residual continuum features are included using 1-5 cosine functions, each with an arbitrary phase, frequency (between 0.5 and 5 periods on the wavelength interval), and normalization (within 0 and $5 \%$ of the continuum level).

Figure 6 presents the reconstruction error (RAVE-true) as a function of the various parameters released in DR2 for the 20,000 simulated spectra. As mentioned before, the rotational velocity will be discussed in another paper, and microturbulence cannot be recovered in the RAVE regime. Therefore, these two parameters are not presented here. Nevertheless, we stress that all six parameters were used in the simulations, the same as was done in the standard pipeline on observed spectra. The left panel represents the spectra with effective temperature below $8000 \mathrm{~K}$ (Ca II lines dominated), while the right panel presents the hotter spectra that are dominated by the hydrogen Paschen lines. The number of simulated spectra in the left panel is $\sim 17,500$ while the right panel contains $\sim 2500$ simulated spectra. This is an effect of the template library, the cool part of the library having a denser grid of spectra than the hot side. Also, a smoothing was applied to the right panel for the visualization, to lower the effect of the noise.

These simulations enable us to assess the expected dependencies of our errors as a function of the various stellar parameters. The main characteristics we observe are as follows.

1. Below $8000 \mathrm{~K}$, there is little dependence of the recovered parameters on $T_{\text {eff }}$ but for $T_{\text {eff }}$ itself with an overestimation that increases as the effective temperature becomes larger.

2. $[\mathrm{M} / \mathrm{H}]$ is the main driver for the errors in the low metallicity regime $([\mathrm{M} / \mathrm{H}] \lesssim-1.0)$ with all parameters but $[\alpha / \mathrm{Fe}]$ being overestimated, while $[\alpha / \mathrm{Fe}]$ is underestimated. This indicates that for the metallicity, the true metal content can only be recovered when both $[\mathrm{M} / \mathrm{H}]$ and $[\alpha / \mathrm{Fe}]$ are considered (see Section 4.2.2).

3. $[\alpha / \mathrm{Fe}]$, as expected, is not properly recovered in the RAVE regime as shown by the upper right panels.

4. $\log g$ is better constrained in hot spectra than in cool spectra. 5. $T_{\text {eff }}$ is systematically underestimated for hot spectra.

The overall accuracy we can expect for the stellar parameters in the RAVE regime then ranges from $200 \mathrm{~K}$ to $500 \mathrm{~K}$ for $T_{\text {eff }}, 0.2$ to $0.5 \mathrm{dex}$ for $\log g$, and 0.1 to $0.4 \mathrm{dex}$ for $[\mathrm{M} / \mathrm{H}]$ (depending on the value of $\alpha$-enhancement) while $[\alpha / \mathrm{Fe}]$ alone is not recovered.

A better understanding of the relations and mutual influences of the errors on the stellar parameters is gained from the correlations between the reconstruction errors. These are presented in Figure 7, where the different behavior of the hot stars and of the cool stars is apparent. The upper triangle presents the correlations between the errors for the cool stars, while the lower triangle shows the correlation for the hot spectra (the lower triangle has been smoothed for visual rendering).

It is clear in this figure that in the cool spectra regime, the errors on the parameter reconstruction are strongly correlated which indicates that an error on one parameter results in errors on the other parameters. There is however an exception for $[\alpha / \mathrm{Fe}]$ which is only anti-correlated to $[\mathrm{M} / \mathrm{H}]$ and not correlated to the

\footnotetext{
25 The spectra used for parameter estimation are RV corrected after a first RV estimation using a reduced set of templates (see Paper I); a better template with proper parameters is then generated using the algorithm and is then used for the final RV calculation.
} 

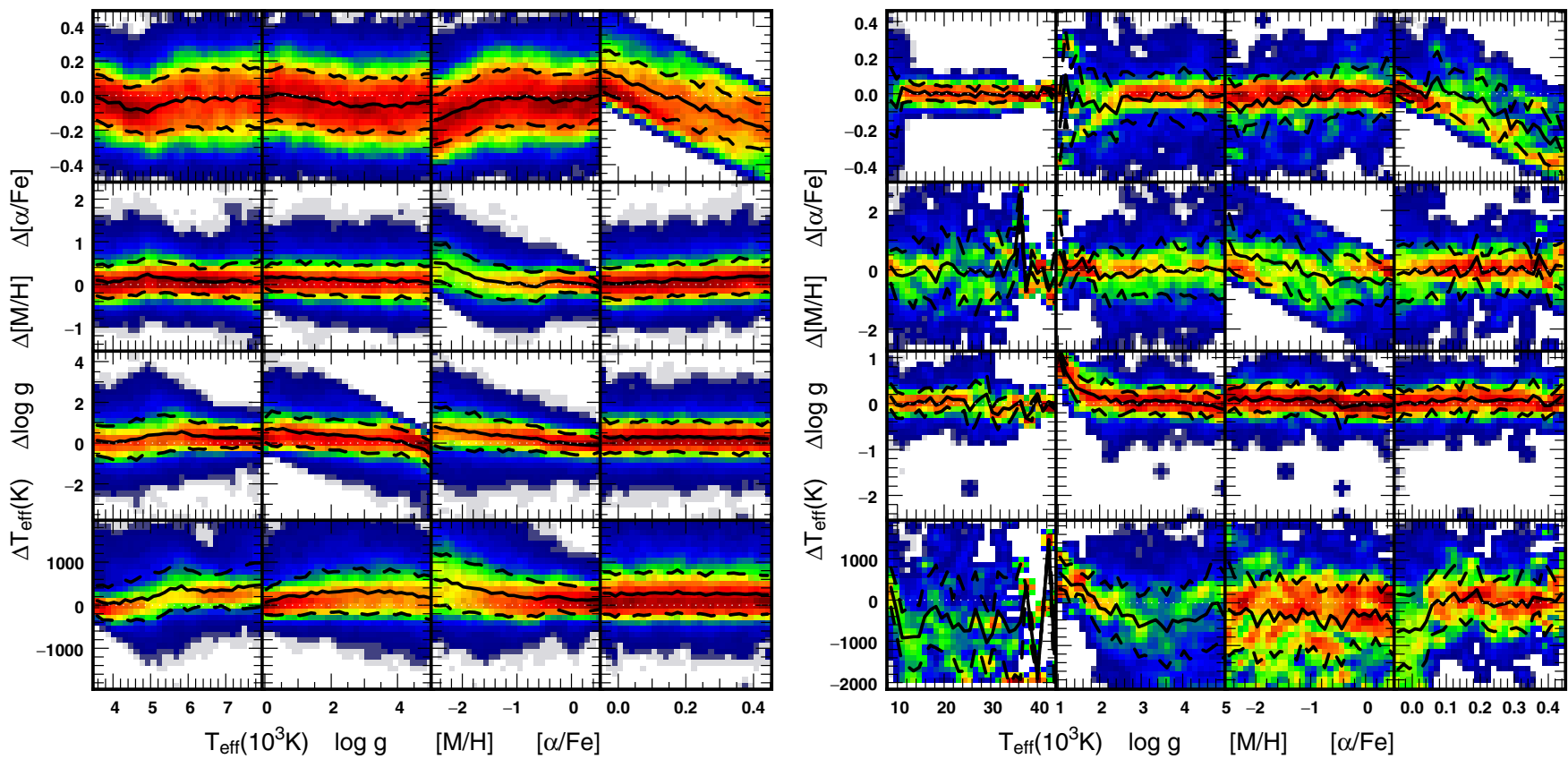

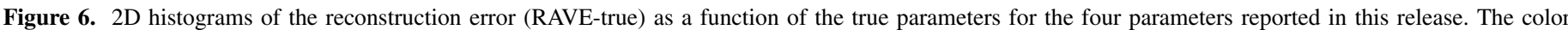

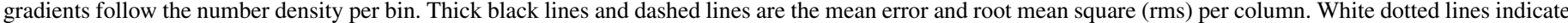
a zero reconstruction error. Left panel: spectra with $T_{\text {eff }}$ below $8000 \mathrm{~K}$. Right panel: spectra with $T_{\text {eff }} \geqslant 8000 \mathrm{~K}$

(A color version of this figure is available in the online journal)

other parameters which further indicates that only a combination of $[\mathrm{M} / \mathrm{H}]$ and $[\alpha / \mathrm{Fe}]$ is recovered, and that these two quantities cannot be uniquely separated.

The situation is different for the hot stars, where the only visible correlation is between $T_{\text {eff }}$ and $[\mathrm{M} / \mathrm{H}]$ and only for large errors on $[\mathrm{M} / \mathrm{H}]$. Otherwise, no correlation is seen, indicating that the system is better constrained. Nevertheless, typical errors for hot stars are larger than for cool stars with similar noise levels.

Overall, the method presented allows us to recover the stellar parameters with a good accuracy knowing that our wavelength interval is small and our resolution is limited $(R \sim 7500)$. The expected correlations between the reconstruction errors for the different parameters are well behaved (simple one-mode correlations) if one is able to distinguish a posteriori the two cases, hot and cool stars.

\subsection{Estimate of the $S / N$}

The initial estimate of the $\mathrm{S} / \mathrm{N}$ comes from comparison of 1D spectra derived from typically five subexposures of a given field (see Paper I for details). This estimate is model independent and readily available for the calculation of $\chi^{2}$ for the RV and stellar parameter determination routines. However, any change of observing conditions during the observing run may contribute to differences of subexposure spectra and therefore render the value of the $\mathrm{S} / \mathrm{N}$ too low. We therefore wrote a procedure which calculates the $\mathrm{S} / \mathrm{N}$ from the final spectrum only. We refer to it as the $\mathrm{S} 2 \mathrm{~N}$ value in the data release, while the one calculated from subexposure variation is labeled SNR.

Line-free regions in observed spectra are very scarce. Moreover, the spectra are quite noisy, so one does not know a priori if an apparently line-free region does not hide weak absorption lines. So it seems obvious that suitable regions should be chosen by comparison of the observed spectrum to the best matching template.
The procedure is as follows.

1. The normalized final observed spectrum (shifted and resampled to the rest frame) is compared with the synthetic library template with the best correlation. The two spectra are not identical for two reasons: noise in the observed spectrum and systematic deviations (due to observational or theoretical computation deficiencies). We want to avoid the latter. The difference between the observed and theoretical spectrum often alternates in sign between consecutive wavelength pixels if it is due to noise. But systematics usually affect several adjacent wavelength bins, so the sign of the difference does not vary so frequently. We therefore decided to use only those pixels for which the difference changes sign from the previous or toward the next adjacent pixel. This selection scheme retains $75 \%$ of all pixels if the reason for variation is just noise. This seems a reasonable price to pay in order to avoid systematics. Note that we impose restrictions only on the sign of the difference, not on its absolute value, so noise properties are not affected.

2. Regions of strong spectral lines are prone to systematic errors. So we discard any pixel for which the flux of the template would be less than 0.9 of the continuum flux. Strong spectral lines span a small fraction of the entire spectral range, except in high temperature objects. The derived $\mathrm{S} / \mathrm{N}$ estimate is representative of the continuum $\mathrm{S} / \mathrm{N}$, but the value generally does not differ by more than $5-10 \%$ from the $\mathrm{S} / \mathrm{N}$ averaged over the whole spectrum.

3. Next, we calculate the difference between the observed and theoretical spectra, and divide it by the theoretical spectrum flux. The final S2N estimate is an inverse of its standard deviation, of course only using the pixels retained in the steps above.

4. The observed spectra we used for the three steps above are shifted to the rest frame and resampled with respect to the original ones given in observed wavelengths. This is 


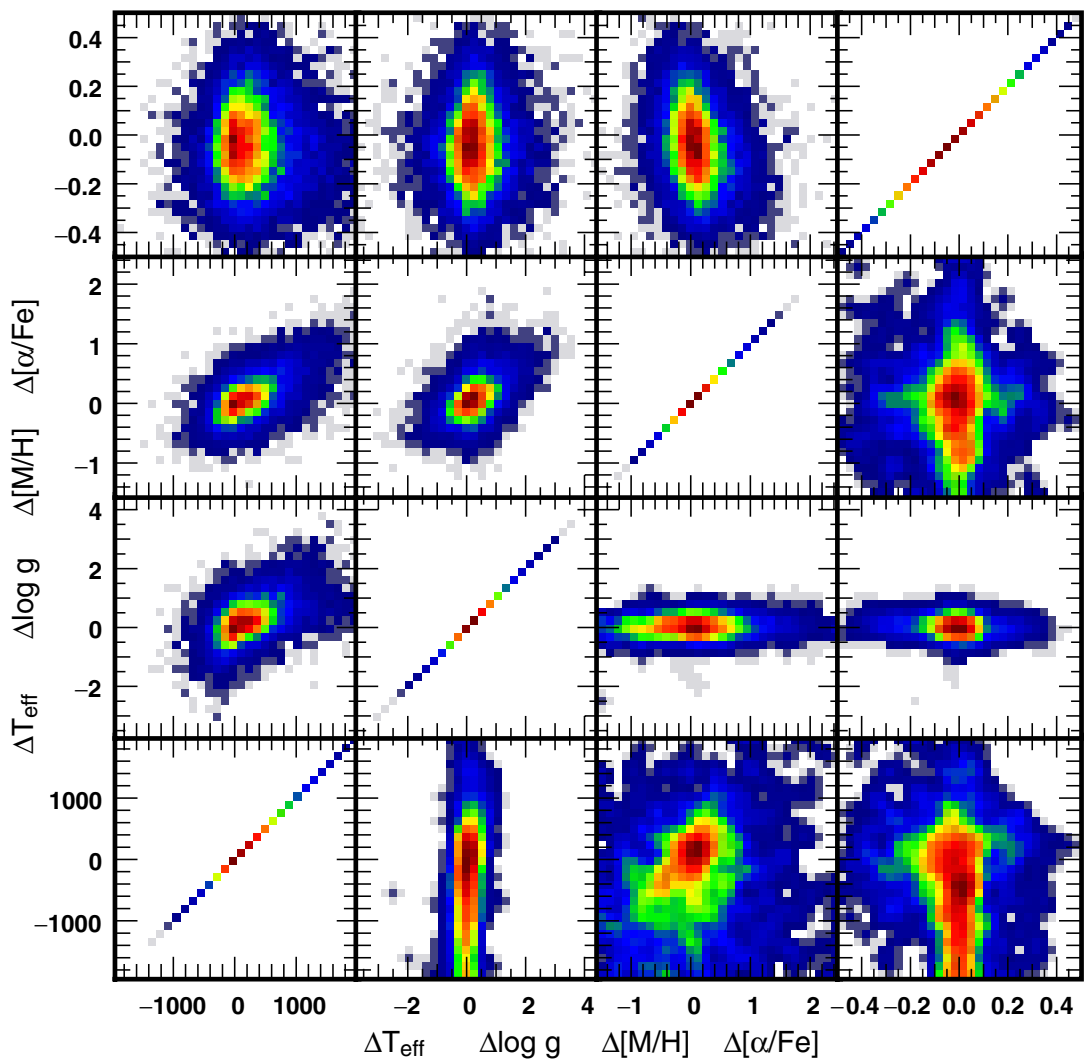

Figure 7. $2 \mathrm{D}$ histograms of the reconstruction errors vs. reconstruction error. The upper triangle is for spectra with effective temperature below $8000 \mathrm{~K}$, the lower triangle for spectra with $T_{\text {eff }}$ above $8000 \mathrm{~K}$. The color coding follows the number density per bin.

(A color version of this figure is available in the online journal)

important, as resampling damps the pixel-to-pixel variation and therefore artificially increases the measured value of the $\mathrm{S} / \mathrm{N}$. So we need to take it into account. The resampled and the original spectra have the same number of pixels, so resampling can be characterized by its average fractionalpixel shift. A zero shift obviously does nothing, but a shift of half a pixel means that the $\mathrm{S} / \mathrm{N}$ estimate measured in previous steps needs to be multiplied by a factor of $1 / \sqrt{2}$. If the shift is a fraction $x$ of the pixel separation, the expression for the damping factor $f_{\mathrm{SN}}=\frac{1-1 / \sqrt{2}}{0.5^{2}}(x-0.5)^{2}+1 / \sqrt{2}$. The $\mathrm{S} / \mathrm{N}$ value calculated by the above three steps needs to be multiplied by this damping factor to obtain the final S2N estimate of the observed spectrum. In the case of RAVE data the fractional shift of pixels at both edges of the spectrum is zero, while the pixels in-between are resampled from an observed nonlinear to a linear increase of wavelength with the pixel number. Because this nonlinearity is also always very similar, the resulting damping factor turns out to be well constrained: $f_{\mathrm{SN}}=0.78 \pm 0.01$. This value is actually very close to 0.805 obtained for a uniform distribution of fractional-pixel shifts $x$ in the $[0,1]$ interval.

The first two points limit the fraction of pixels used in the S2N estimate to $46 \% \pm 6 \%$. This is true also for hot stars, so the selection outlined above does not seem to be too constraining. Note that step 4 means that the S2N values are lower than the ones calculated by, e.g., the splot package of IRAF, because the latter does not take into account the effects of resampling. The SNR estimate is very sensitive to variations of atmosphere transparency and instrumental effects during the observing sequence while the $\mathrm{S} 2 \mathrm{~N}$ is not. So $\mathrm{S} 2 \mathrm{~N}$ values are similar to the SNR ones, with the average value of S2N being $\sim 33 \%$ higher. We propose to use the $\mathrm{S} 2 \mathrm{~N}$ as the final $\mathrm{S} / \mathrm{N}$ estimate for the spectrum. So the quantity $\mathrm{S} / \mathrm{N}$ below always refers to the $\mathrm{S} 2 \mathrm{~N}$ value.

Figure 8 plots S2N as a function of the DENIS I magnitude and average number of counts per pixel. The latter was calculated in the central part of the spectrum $(8449.77 \AA \leqslant$ $\lambda \leqslant 8746.84 \AA$ ). The straight line in the magnitude graph (Figure $8(\mathrm{a})$ ) follows the relation

$$
\mathrm{S} / \mathrm{N}=10^{-0.2\left(\text { IDENIS }_{\mathrm{D}}-19.1\right)},
$$

while the one in Figure 8(b) is obtained by combining it with Equation (1). The constant term in Equation (18) is the mode of the magnitude-corrected $\mathrm{S} / \mathrm{N}$ distribution. The magnitude graph shows that the $\mathrm{S} / \mathrm{N}$ can be predicted from DENIS I magnitude with an average error of $\approx \pm 50 \%$. The dependence of $\mathrm{S} / \mathrm{N}$ on the count level is much better determined, with a dispersion of the central ridge of only $\pm 15 \%$. The difference is due to an uneven transparency of the Earth's atmosphere and of optical fibers which have a stronger effect on the magnitude graph. Sky background as well as light scattered within the spectrograph is of increasing relative importance for faint objects. They cause the deviation from a straight line seen in both panels at faint count or magnitude levels.

\section{DATA QUALITY}

\subsection{RV Accuracy}

The distribution of the internal RV errors is presented in Figure 9. These are the estimated uncertainties of fitting a 

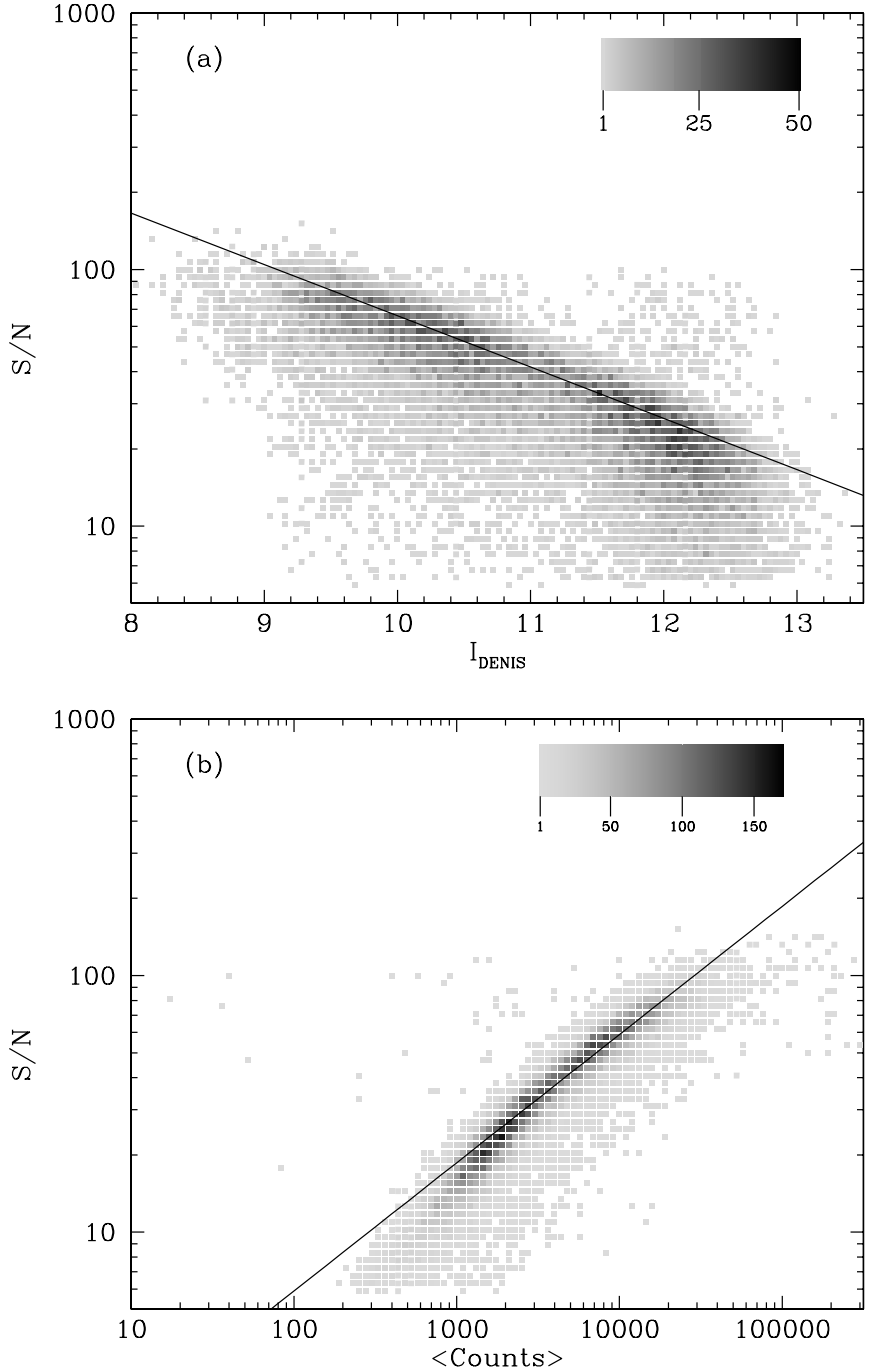

Figure 8. Signal-to-noise ratio (S2N) as a function of the DENIS $I$ magnitude (a) and average level of counts per wavelength bin per hour of exposure time (b). Shades of gray mark the number of spectra in a given bin, as explained in the key. The straight line in the top panel follows relation (18), while the one in the bottom panel follows from Equations (1) and (18).

Table 2

Internal RV Errors

\begin{tabular}{lcc}
\hline \hline Dataset & $\begin{array}{c}\text { Peak } \\
\left(\mathrm{km} \mathrm{s}^{-1}\right)\end{array}$ & $\begin{array}{c}\text { Average } \\
\left(\mathrm{km} \mathrm{s}^{-1}\right)\end{array}$ \\
\hline Spectra of normal stars new to DR2 & 0.9 & 2.0 \\
Spectra of all stars new to DR2 & 0.9 & 2.5 \\
Spectra of all stars in DR1 & 1.7 & 2.3 \\
All & 1.6 & 2.3 \\
\hline
\end{tabular}

Note. Peak value refers to the histogram in Figure 9.

parabola to the top of the correlation peak (Paper I). The top panel shows the histogram of the RV error in $0.1 \mathrm{~km} \mathrm{~s}^{-1}$ bins, while the bottom panel is the cumulative distribution. Results for the spectra new to this data release and for the ones from Paper I are shown separately. In the former case, we also add the results for spectra for which we are publishing the values of stellar parameters (see below). These are spectra of a sufficiently high quality and without peculiarities (binarity, emission lines, etc.). Table 2 summarizes the values of the most probable and average internal velocity errors.
Table 3

Datasets Used to Check the RV Accuracy

\begin{tabular}{lrcc}
\hline \hline Reference dataset & $N$ & $\begin{array}{c}\langle\Delta \mathrm{RV}\rangle \\
\left(\mathrm{km} \mathrm{s}^{-1}\right)\end{array}$ & $\begin{array}{c}\sigma(\Delta \mathrm{RV}) \\
\left(\mathrm{km} \mathrm{s}^{-1}\right)\end{array}$ \\
\hline GCS & 144 & 0.34 & 1.83 \\
Sophie observations & 33 & 0.63 & 1.18 \\
Asiago observations & 21 & -0.71 & 1.09 \\
ELODIE observations & 15 & 0.07 & 1.32 \\
All & 213 & 0.26 & 1.68 \\
Last three datasets & 69 & 0.10 & 1.30 \\
\hline
\end{tabular}

Note. $\triangle \mathrm{RV}$ is the difference between radial velocities derived by RAVE and those from the reference dataset.

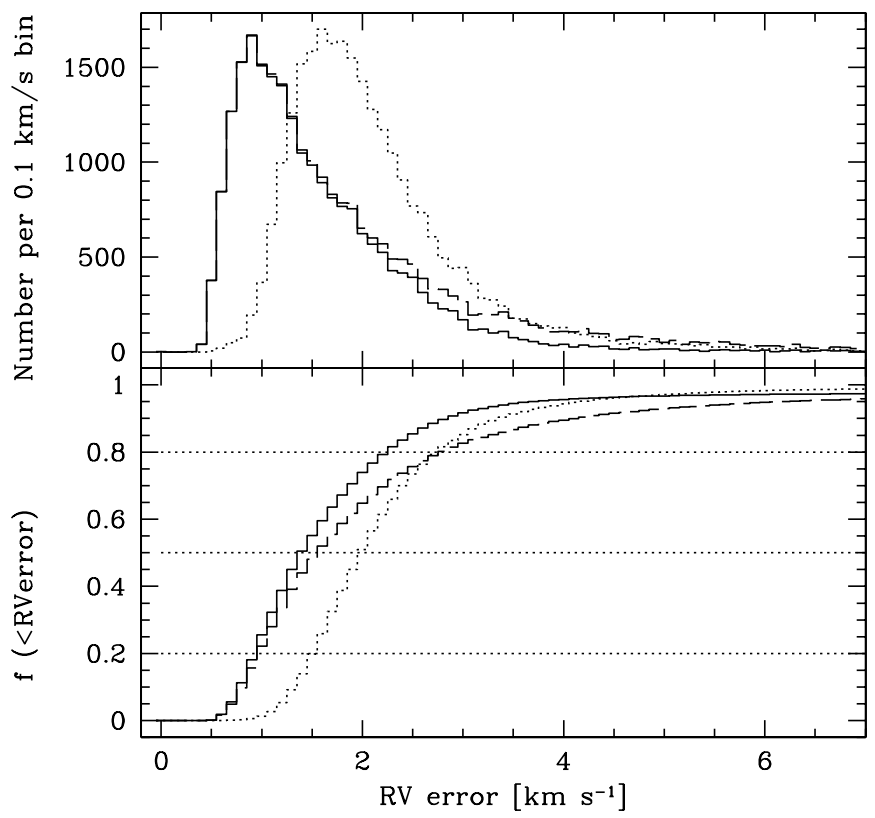

Figure 9. Top panel: distribution of the internal RV error. The solid line is for normal stars which also have their values of stellar parameters published. The dashed line is for all stars new to the present data release. The dotted histogram is stars from the first data release. Bottom panel: fraction of RAVE targets with a RV error lower than a given value. The dotted lines indicate limits of $20 \%$, $50 \%$, and $80 \%$. The line types are the same as in the top panel.

The blue light blocking filter, which cuts the second-order light and was used for data new to this release, clearly improves the match between theoretical templates and observed spectra. This is mostly a consequence of the more accurate flat-fielding of a rather narrow spectral range of the first-order light, compared to a mix of relative contributions of the first- and the secondorder spectra which emphasizes any differences in the color temperature between the star and the calibration lamp. Also, the level of the continuum is much easier to determine if the blocking filter is used. The most probable value of the internal velocity error is $0.9 \mathrm{~km} \mathrm{~s}^{-1}$ for the data new to this data release, compared to $1.7 \mathrm{~km} \mathrm{~s}^{-1}$ in Paper I. On the other hand, the possibility of a better match also increases the chances to identify any types of peculiarities. So there is a rather notable tail of large internal velocity errors if we consider all data new to this data release (dashed line in Figure 9). If only normal stars are plotted (solid line in Figure 9), large velocity errors are much less common. This is also reflected in the average errors reported in Table 2.

Internal velocity errors are useful, but they do not include possible systematic effects. As mentioned in Section 3.2, the 


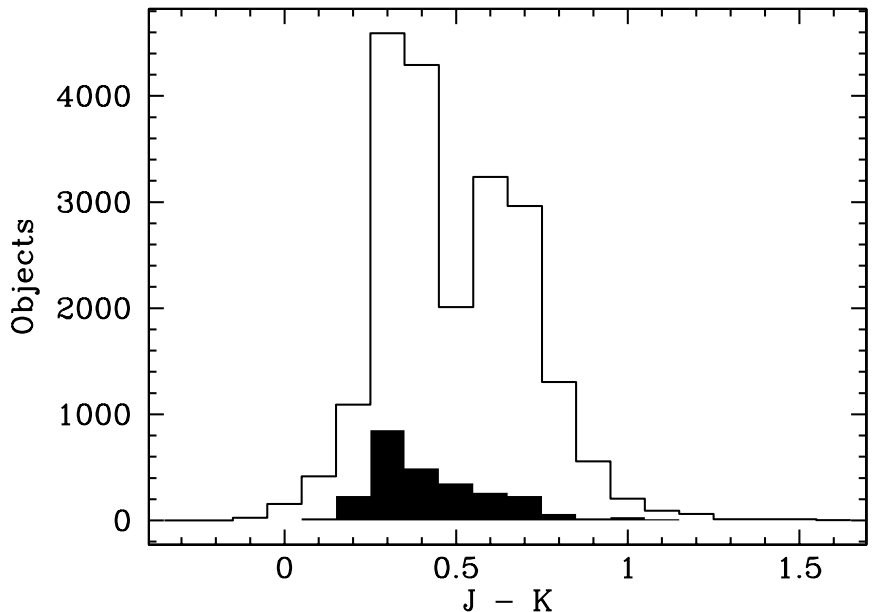

Figure 10. $(J-K)_{2 \mathrm{MASS}}$ colors for the second data release (solid line) and for reference stars used to check the RV accuracy (filled histogram, its values multiplied by 10). Most reference stars, especially those from the GCS, are yellow dwarfs with only a small fraction of red giants.

reported radial velocities do not allow for shifts due to nonvanishing convective motions in the stellar atmosphere and for gravitational redshift of the light leaving the stellar surface. This is the case also with other spectroscopically determined radial velocities. We compared RAVE radial velocities with those obtained from external datasets. A total of 255 stars from four different datasets were used to assess the accuracy of radial velocities of stars new to this data release. From these, 213 stars turn out to have normal spectra without emission lines, strong stellar activity, or stellar multiplicity and have RV errors smaller than $5 \mathrm{~km} \mathrm{~s}^{-1}$, so that they are retained for further analysis. They include 144 stars from the Geneva Copenhagen Survey (GCS), and three datasets observed specifically to check RAVE radial velocities: 33 stars were observed with the Sophie and 15 with the ELODIE spectrograph at the OHP, and 21 stars with the echelle spectrograph at the Asiago observatory. Stars observed in Asiago span the whole range of colors, while most other datasets and especially GCS focus on yellow dwarfs. The whole survey includes a larger number of red stars (Figure 10) which are mostly giants, as can be seen from temperature-gravity distributions derived by RAVE for the whole survey (Figure 22). A smaller fraction of giants in the reference datasets does not present a real problem, as radial velocities for giants tend to be more accurate than for dwarfs.

A comparison of radial velocities obtained by RAVE and by the reference datasets is presented in Figure 11 and summarized in Table 3. $N$ is the number of objects in each dataset, $\langle\Delta \mathrm{RV}\rangle$ is the mean of differences between RAVE and reference measurements and $\sigma(\Delta \mathrm{RV})$ is their standard deviation. We note that mean zero-point offsets are non-zero and of different sizes and signs for separate datasets. So the difference in the zero point is likely due to a different zero-point calibration of each instrument. Most of the reference stars are taken from the GCS. So the large number of dwarfs from the GCS also drive the final value of the zero-point offset and its dispersion. If one omits these, the mean zero-point difference is only $0.1 \mathrm{~km} \mathrm{~s}^{-1}$ and the dispersion $(\sigma(\Delta \mathrm{RV}))$ is $1.30 \mathrm{~km} \mathrm{~s}^{-1}$. These estimates neglect the intrinsic measurement errors of each reference dataset. A typical error of $0.7 \mathrm{~km} \mathrm{~s}^{-1}$ and a zero-point offset of $0.3 \mathrm{~km} \mathrm{~s}^{-1}$ for the GCS suggest that the RV error of RAVE is $\sim 1.3 \mathrm{~km} \mathrm{~s}^{-1}$. This is also the value derived from the other datasets. Figure 12 shows that the standard deviation stays within $\sim 2 \mathrm{~km} \mathrm{~s}^{-1}$ even

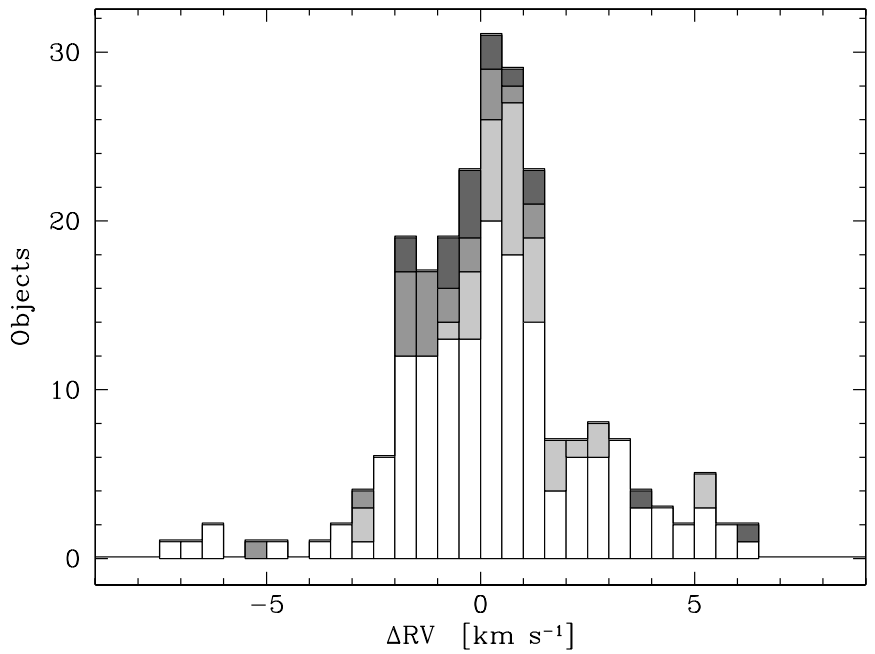

Figure 11. Difference of radial velocities as derived by RAVE and by the reference instruments. The solid histogram is for all objects with colored rectangles belonging to individual external datasets: the GCS (white), Sophie (light gray), Asiago (dark gray), and ELODIE (black).

at low values of $\mathrm{S} / \mathrm{N}$. This value decreases to $\sim 1.5 \mathrm{~km} \mathrm{~s}^{-1}$ if one omits the stars from the GCS dataset.

Most of the stars in external datasets are dwarfs with a metallicity close to the solar one. The midpoint of $|\Delta R V|$ stays at $\sim 1.2 \mathrm{~km} \mathrm{~s}^{-1}$ for temperatures lower than $5800 \mathrm{~K}$, increasing to $\sim 2 \mathrm{~km} \mathrm{~s}^{-1}$ for stars with $6800 \mathrm{~K}$. There is no significant variation of the $\mathrm{RV}$ difference with metallicity in the range $-0.5<[\mathrm{M} / \mathrm{H}]<0.3$ covered by the external datasets.

One can also use repeated observations of RAVE stars to assess the internal consistency of the measurements. Section 4.4 shows that RV from a pair of measurements of a single star differ by $\leqslant 1.80 \mathrm{~km} \mathrm{~s}^{-1}$ in $68.2 \%$ of the cases. This corresponds to an error of $1.3 \mathrm{~km} \mathrm{~s}^{-1}$ for a single measurement.

We conclude that the typical RV error for data new to this data release is $\lesssim 2 \mathrm{~km} \mathrm{~s}^{-1}$. For the measurements with a high value of $\mathrm{S} / \mathrm{N}$, the error is only $1.3 \mathrm{~km} \mathrm{~s}^{-1}$ with a negligible zero-point error.

\subsection{Accuracy of Stellar Parameters}

For the vast majority of the stars in this data release, there is no prior spectroscopic information available. Some photometric information is available (see Section 5.2) but after a detailed investigation we concluded that this external information is not of sufficient quality to be used as a prior on any of the stellar parameters. Unknown extinction presents a further problem. This situation is expected to continue until high-quality multi-epoch photometry becomes available for the southern sky from the SkyMapper project (Keller et al. 2007). RAVE is therefore the first large spectroscopic survey to use only spectroscopic data to derive the values of stellar parameters. So it is appropriate to make a detailed check of the results with external datasets coming from both the literature and our own custom observations.

\subsubsection{External Datasets}

RAVE stars are generally too faint to have data available in the literature, so we obtained a separate set of RAVE observations of stars from three reference sets in the literature. In addition, we obtained custom observations of regular RAVE targets with two Northern Hemisphere telescopes, at Observatory in Asiago and at Apache Point Observatory (APO). In the coming 


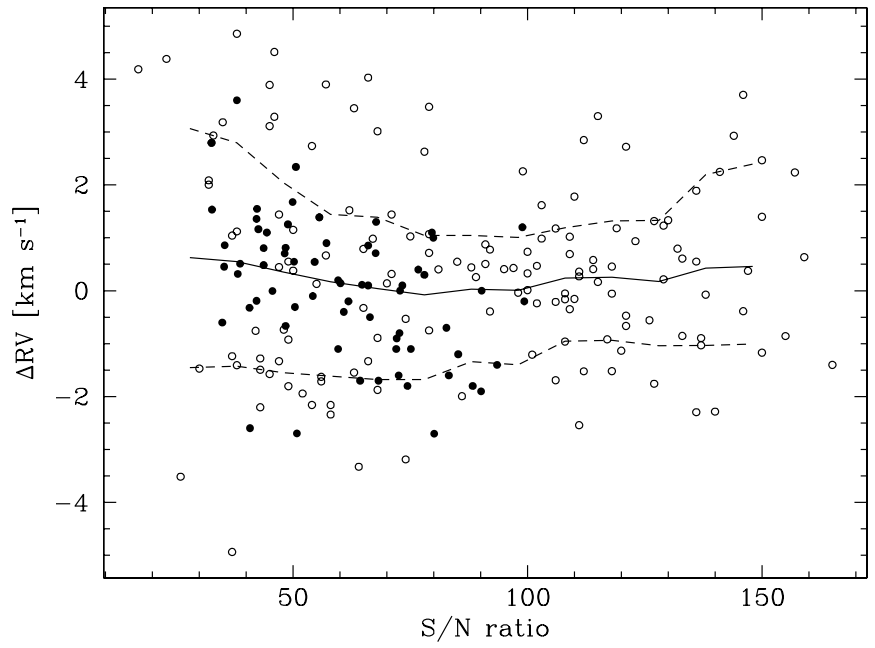

Figure 12. Difference of radial velocities as derived by RAVE and by the reference instruments, as a function of the $\mathrm{S} / \mathrm{N}$ of the RAVE observation. Dots depict individual measurements, the solid line is a running mean (with a boxcar smoothing of $\pm 15 \mathrm{in} \mathrm{S} / \mathrm{N}$ ). Similarly, the area between the dashed lines includes $68.2 \%$ of the measurements, i.e., $\pm \sigma$. The open symbols are measurements of the GCS stars, while the filled ones mark the other three reference datasets.

months, we plan to expand the comparison using the observing time allocated at UCLES at Siding Spring and at ESO. Here we describe the currently available datasets which contain altogether 331 stars. In all cases the corresponding RAVE observations were obtained and processed in the same way as for the other stars in this data release.

The ARC echelle spectrograph at the APO $3.5 \mathrm{~m}$ telescope was used to observe 45 RAVE stars. These spectra cover the entire optical wavelength range (3500-10000 $⿱$ ) in 107 orders with an effective resolving power of about 35,000 . The spectra were extracted using standard IRAF routines incorporating bias and scattered light removal plus flat-field corrections. The wavelength calibration was obtained using ThAr hollow cathode lamps. Temperature and gravity were derived by a least squares fit to the same library of stellar spectra (Munari et al. 2005a) as used for the RAVE catalog, but using a procedure (Munari et al. 2005) independent of the RAVE method described in Section 3.3. The results are consistent with those resulting from the analysis using the excitation temperature and equivalent widths of $\mathrm{Fe} \mathrm{I}$ and $\mathrm{Fe}$ II lines to derive iron abundance, temperature, and gravity (Fulbright et al. 2006, 2007). The metallicity was derived by both the least-squares fit of the whole spectrum and by the method based on equivalent widths of the $\mathrm{Fe}$ lines. The latter yields an iron abundance, but the metallicity can be calculated assuming that the $[\alpha / \mathrm{Fe}]$ ratio, which influences the even- $Z$ elements between $\mathrm{O}$ and $\mathrm{Ti}$, increases linearly from zero for stars with $[\mathrm{Fe} / \mathrm{H}]=0$ to +0.3 for stars with $[\mathrm{Fe} / \mathrm{H}]$ $=-1$, and stays constant outside these ranges. In Table 10, we list the temperature and gravity as derived by the least-squares fit method, and metallicity from the Fe line method.

The echelle spectrograph at the $1.8 \mathrm{~m}$ telescope, operated by INAF Osservatorio Astronomico di Padova on top of Mt. Ekar in Asiago was used to observe 24 RAVE stars. These spectra cover the range from 3300 to $7300 \AA$, but the analysis was limited to the three echelle orders around $5200 \AA$ with the highest signal. The resolving power was around 20,000. The spectra were carefully treated for scattered light, bias, and flat field, and reduced using standard IRAF routines. They were analyzed with the same least-squares procedure as the APO data. The results are given in Table 11 .
Table 4

Datasets Used to Check Stellar Parameters

\begin{tabular}{lcccc}
\hline \hline Reference dataset & $N$ & $T_{\text {eff }}$ & $\log g$ & {$[\mathrm{M} / \mathrm{H}]$} \\
\hline APO & 45 & $\sqrt{ }$ & $\sqrt{ }$ & $\sqrt{ }$ \\
Asiago echelle & 24 & $\sqrt{ }$ & $\sqrt{ }$ & $\sqrt{ }$ \\
Soubiran \& Girard catalog & 49 & $\sqrt{ }$ & & $\sqrt{ }$ \\
M67 members & 12 & & & $\sqrt{ }$ \\
GCS & 211 & $\sqrt{ }$ & & \\
\hline
\end{tabular}

Table 5

Zero-Point Offsets and Dispersions of the Differences Between RAVE and Reference Stellar Parameter Values

\begin{tabular}{lrc}
\hline \hline Parameter & Zero point & Dispersion \\
\hline Temperature (without GCS dataset) & $-7 \pm 18 \mathrm{~K}$ & $188 \mathrm{~K}$ \\
Temperature (with GCS dataset included) & $53 \pm 14 \mathrm{~K}$ & $238 \mathrm{~K}$ \\
Gravity & $-0.06 \pm 0.04$ & 0.38 \\
Uncalibrated metallicity $([\mathrm{m} / \mathrm{H}])$ & $-0.26 \pm 0.03$ & 0.37 \\
Calibrated metallicity $([\mathrm{M} / \mathrm{H}])$ & $0.0 \pm 0.02$ & 0.18 \\
\hline
\end{tabular}

Note. In the case of metallicity, the reference values are those obtained by the measurement of equivalent widths of absorption lines in the APO observations, as derived from the Soubiran \& Girard (2005) catalog and from the adopted metallicity of M67.

The RAVE spectrograph was used to observe three additional sets of stars with parameters known from the literature. We observed 60 stars from the Soubiran \& Girard (2005) catalog and obtained 49 spectra useful to check the metallicity and the temperature values. The reported gravity values were not used for checks as the catalog does not estimate their accuracy. Soubiran \& Girard (2005) do not report metallicity, so its value was derived from a weighted sum of quoted element abundances of $\mathrm{Fe}, \mathrm{O}, \mathrm{Na}, \mathrm{Mg}, \mathrm{Al}, \mathrm{Si}, \mathrm{Ca}, \mathrm{Ti}$, and $\mathrm{Ni}$, assuming solar abundance ratios from Anders \& Grevesse (1989), in accordance with classical Kurucz models. The choice of a reference solar abundance model is not critical. Newer solar abundance scales introduce only a small shift in the mean metallicity of the Soubiran \& Girard (2005) stars if compared to typical errors of RAVE observations: $\Delta[\mathrm{M} / \mathrm{H}]=-0.002$ for solar abundances given by Grevesse \& Sauval (1998) and $\Delta[\mathrm{M} / \mathrm{H}]=-0.005$ for Asplund et al. (2006) solar abundances. The standard deviation of metallicities, derived from new compared to classical abundances, is 0.005 for Grevesse \& Sauval (1998) and 0.012 for Asplund et al. (2006). The parameter values as derived from the literature and from the RAVE spectra are listed in Table 12.

We also observed 12 members of the M 67 cluster (Table 13) for which we adopted the metallicity of +0.01 . This value of metallicity is a weighted sum of its modern metallicity determinations (Randich et al. 2006 and references therein). Finally, Table 14 reports on the comparison of temperatures for 201 stars from the GCS (Nordström et al. 2004). This catalog does not include metallicities but only iron abundances. The two values are not identical, so a comparison on a star-by-star basis could not be made (but see below for a general comparison of the two values).

Table 4 summarizes the properties of individual datasets. $N$ is the number of stars in a given dataset and the $\sqrt{ }$ sign marks parameters that could be checked. Temperatures, gravities and metallicities of stars in these datasets are plotted in Figure 13. The values are those determined from RAVE spectra, as some parameter values are not known for the datasets from the literature. The distributions of external dataset objects in the temperature-gravity-metallicity space can be compared to those of the whole data release (Figure 22). 
Table 6

Number of Entries with a Given Stellar Type in This Data Release

\begin{tabular}{|c|c|c|c|c|c|}
\hline \multirow[t]{2}{*}{ Type } & \multirow[t]{2}{*}{ SpectraFLAG } & \multicolumn{2}{|c|}{ Published } & \multicolumn{2}{|c|}{ Number of } \\
\hline & & RVs & Parameters & Spectra & Stars \\
\hline All entries with RVs & & $\sqrt{ }$ & & 51,829 & 49,327 \\
\hline Entries with normal-type spectra & & $\sqrt{ }$ & $\sqrt{ }$ & 22,407 & 21,121 \\
\hline Any type without parameters & & $\sqrt{ }$ & & 29,422 & 28,747 \\
\hline Emission line & e & $\sqrt{ }$ & & 140 & 136 \\
\hline Double-lined binaries & $\mathrm{p}$ & $\sqrt{ }$ & & 135 & 132 \\
\hline Peculiar & $\mathrm{x}$ & $\sqrt{ }$ & & 86 & 75 \\
\hline
\end{tabular}

\subsubsection{Comparison of External and RAVE Parameter Values}

The first property to check is the consistency of values derived by the RAVE pipeline with those from the reference datasets. Table 5 lists mean offsets and dispersions around the mean for individual stellar parameters.

The temperature shows no offsets when the reference sets of echelle observations at APO and Asiago are used, together with our observations of Soubiran \& Girard (2005) stars. However if the GCS dataset is included the RAVE temperatures appear too hot on average, and the dispersion is also increased. We believe that this is a consequence of somewhat larger errors introduced by the photometric determination of the temperature in the GCS and not a consequence of errors of the RAVE pipeline. Gravity shows a negligible offset and a dispersion of 0.4 dex. However, the metallicity as derived by the RAVE pipeline (in Table 5, we refer to it as "uncalibrated") appears to have a significant offset. The values derived by the RAVE pipeline are generally more metal poor than those obtained by the measurement of equivalent widths of absorption lines in APO observations, as derived from the Soubiran \& Girard (2005) catalog, and also compared to the metallicity of M67. So it seems worthwhile to explore the possibility of a calibration that would make metallicities derived by RAVE consistent with the values in these reference datasets.

\subsubsection{Calibrating Metallicity}

The RAVE pipeline derives metallicity as any other parameter, i.e., by a penalized $\chi^{2}$ technique finding an optimal match between the observed spectrum and the one constructed from a library of pre-computed synthetic spectra. The results match even for the metallicity if a similar analysis method is used. This is demonstrated in Figure 14. The results of the analysis using an independent $\chi^{2}$ procedure (Munari et al. 2005) yield metallicities which are entirely consistent with the RAVE pipeline results (mean offset of $0.04 \pm 0.02$ dex and a standard deviation of 0.17 dex). RAVE metallicities as derived from the RAVE pipeline are part of a self-consistent native RAVE system of stellar parameters which is tied to a $\chi^{2}$ analysis using a library of Kurucz template spectra. The system is unlikely to change in the future. So metallicities, as derived by the pipeline, are also a part of the final data release.

However other spectral methods, which derive metallicities from the strengths of individual spectral lines and not from a $\chi^{2}$ match of synthetic and observed spectra, do not yield results so consistent with those of the RAVE pipeline. Figure 15 shows some obvious trends:

1. the difference between the RAVE and the reference metallicity increases with an increased $\alpha$-enhancement, in the sense that RAVE values become too metal poor;

2. the difference is also larger at lower metallicities;

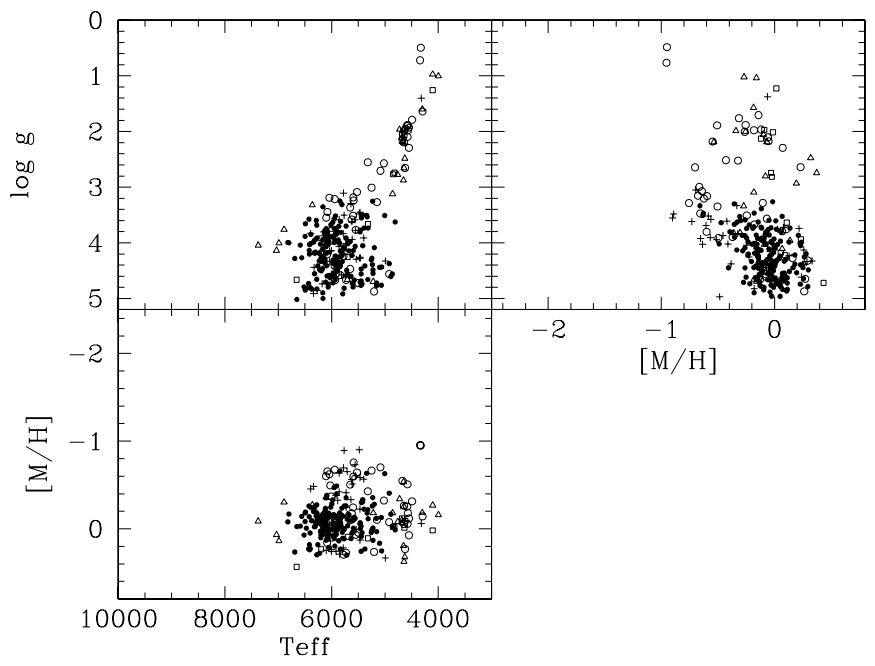

Figure 13. Objects from external datasets on the temperature-gravitymetallicity wedge using the values determined from the RAVE spectra. Symbols code individual datasets which were used to check the values of stellar parameters: GCS $(\bullet)$, APO (०), Soubiran (+), M67 (ם), and Asiago ( $\triangle$ ).

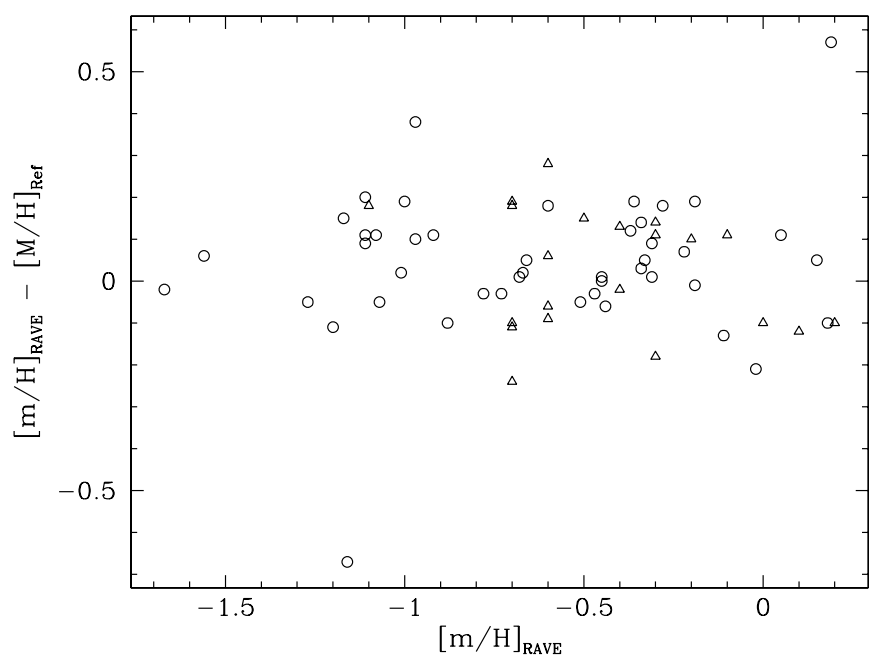

Figure 14. Comparison of metallicities derived by the RAVE pipeline with those from an alternative $\chi^{2}$ analysis (Munari et al. 2005) used as a reference. The circles are the results for the APO stars, while the triangles are the ones for the Asiago stars (Table 11). Metallicities derived by the two methods match very well. The mean values of the metallicity difference do not exceed 0.1 dex in the studied range of $-1.7<[\mathrm{M} / \mathrm{H}]<0.2$.

3. the difference is larger for giants than for main-sequence stars, though the variation is much weaker than for $\alpha$ enhancement or metallicity;

4. the difference does not seem to depend on temperature. 
Table 7

Number and Fraction of RAVE Database Entries with a Counterpart in the Photometric Catalogs

\begin{tabular}{lcccccc}
\hline \hline \multirow{2}{*}{ Catalog name } & \multirow{2}{*}{$\begin{array}{c}\text { Number of } \\
\text { entries }\end{array}$} & \multirow{2}{*}{ \% of entries } & \multicolumn{3}{c}{$\%$ with quality flag } \\
\cline { 3 - 6 } & with counterpart & $A$ & $B$ & $C$ & $D$ \\
\hline 2MASS & 51,813 & $99.97 \%$ & $99.6 \%$ & $0 \%$ & $0 \%$ & $0.4 \%$ \\
DENIS & 40,106 & $77.4 \%$ & $73.7 \%$ & $23.5 \%$ & $2.3 \%$ & $0.5 \%$ \\
USNO-B & 51,466 & $99.3 \%$ & $99.2 \%$ & $0.5 \%$ & $0 \%$ & $0.3 \%$ \\
\hline
\end{tabular}

Table 8

Summary of Proper Motion Sources and their Average and 90\% Errors

\begin{tabular}{lccccc}
\hline \hline $\begin{array}{l}\text { SPM } \\
\text { flag }\end{array}$ & $\begin{array}{c}\text { Catalog } \\
\text { name }\end{array}$ & $\begin{array}{c}\text { Number of } \\
\text { entries }\end{array}$ & $\begin{array}{c}\text { Fraction } \\
\text { of entries }\end{array}$ & $\begin{array}{c}\text { Average } \\
\text { PM error } \\
\left(\mathrm{mas} \mathrm{yr}^{-1}\right)\end{array}$ & $\begin{array}{c}\text { 90\% error } \\
\left(\mathrm{mas} \mathrm{yr}^{-1}\right)\end{array}$ \\
\hline 0 & No proper motion & 74 & $0.1 \%$ & & \\
1 & Tycho-2 & 879 & $1.7 \%$ & 2.9 & 4.0 \\
2 & SSS & 3427 & $6.6 \%$ & 23.7 & 31.7 \\
3 & STARNET 2.0 & 31,739 & $61.2 \%$ & 3.3 & 4.6 \\
4 & 2MASS+GSC 1.2 & 62 & $0.1 \%$ & 18.7 & 26.1 \\
5 & UCAC2 & 15,047 & $29.0 \%$ & 6.7 & 11.1 \\
$1-5$ & All with proper motion & 51,154 & $98.7 \%$ & 5.7 & 10.6 \\
\hline
\end{tabular}
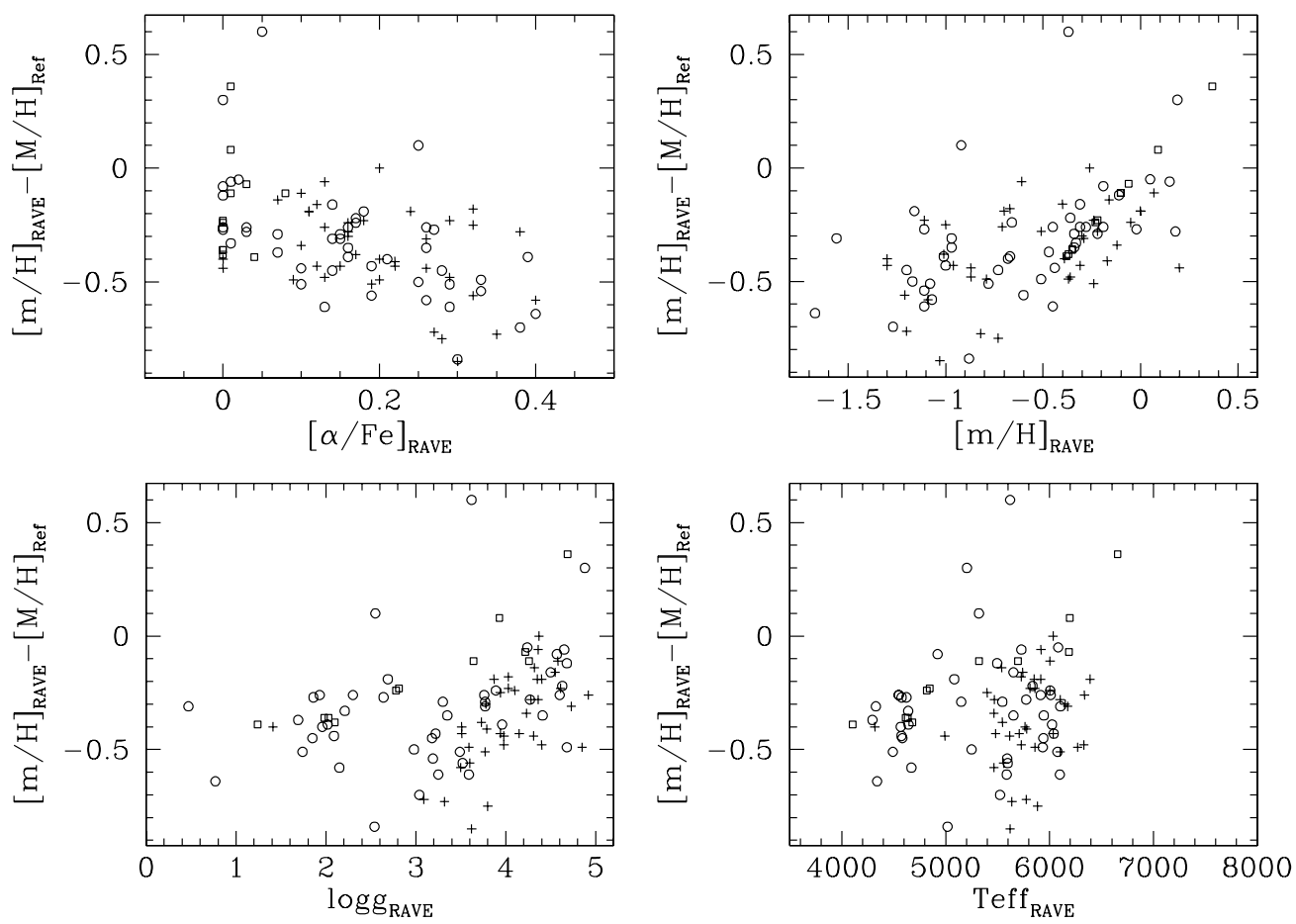

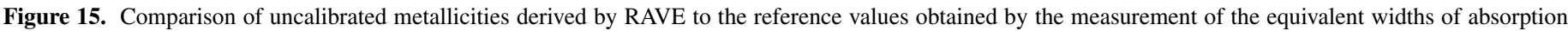
lines or from the literature. Symbol types distinguish between the reference datasets and are the same as in Figure 13.

The aim of this section is to provide a calibration relation that transforms the uncalibrated metallicities, derived by the $\chi^{2}$ method, to the calibrated ones, which are in line with the metallicity system of the above-mentioned datasets. The trends can be represented with a linear relationship; there is no indication of quadratic terms. So we assume that the calibrated metallicity $[\mathrm{M} / \mathrm{H}]$ is given by the relation

$$
[\mathrm{M} / \mathrm{H}]=c_{0}[\mathrm{~m} / \mathrm{H}]+c_{1}[\alpha / \mathrm{Fe}]+c_{2} \log g+c_{3} T_{\text {eff }}+c_{4},
$$

where all parameters on the right refer to the values derived by the RAVE pipeline (Section 3.3) and $c_{i}$ are constants. Figure 15 contains a few outliers, so there is a danger that the fit is driven by these points and not by general trends. The fit is therefore performed twice and after the first fit we reject 5\% of the most deviating points. Such a clipping does not decrease the number of calibration points significantly; still it effectively avoids outliers.

It is not obvious whether all parameters in Equation (19) need to be used. So we tested a range of solutions, using between one and five free parameters. It turns out that the main parameters are metallicity, $\alpha$-enhancement, and gravity, while for the temperature parameter $\left(c_{3}\right)$ improvement of the goodness of fit is not significant. Also, the calibrating datasets cover a limited range in temperature, so this parameter is not sampled over its whole physical span. So we decided not to use 
Table 9

Catalog Description

\begin{tabular}{|c|c|c|c|c|c|}
\hline $\begin{array}{l}\text { Column } \\
\text { number }\end{array}$ & $\begin{array}{c}\text { Character } \\
\text { range }\end{array}$ & Format & Units & Symbol & Description \\
\hline 1 & $1-16$ & A16 & $\ldots$ & Name & Target designation \\
\hline 2 & $18-29$ & F12.8 & $\operatorname{deg}$ & RAdeg & Right ascension (J2000.0) \\
\hline 3 & $31-42$ & F12.8 & deg & DEdeg & Declination (J2000.0) \\
\hline 4 & $44-52$ & F9.5 & $\operatorname{deg}$ & GLON & Galactic longitude \\
\hline 5 & $54-61$ & F9.5 & $\operatorname{deg}$ & GLAT & Galactic latitude \\
\hline 6 & $64-70$ & F7.1 & $\mathrm{km} \mathrm{s}^{-1}$ & HRV & Heliocentric RV \\
\hline 7 & $72-77$ & F6.1 & $\mathrm{km} \mathrm{s}^{-1}$ & eHRV & HRV error \\
\hline 8 & $79-84$ & F6.1 & mas $\mathrm{yr}^{-1}$ & pmRA & Proper motion RA \\
\hline 9 & $86-91$ & F6.1 & $\operatorname{mas} \mathrm{yr}^{-1}$ & epmRA & Error proper motion RA \\
\hline 10 & $93-98$ & F6.1 & $\operatorname{mas} \mathrm{yr}^{-1}$ & pmDE & Proper motion DE \\
\hline 11 & $100-105$ & F6.1 & mas $\mathrm{yr}^{-1}$ & epmDE & Error proper motion DE \\
\hline 12 & $107-107$ & I1 & $\ldots$ & Spm & Source of proper motion (1) \\
\hline 13 & $109-113$ & F5.2 & mag & Imag & Input catalog I magnitude \\
\hline 14 & $115-122$ & A8 & $\ldots$ & Obsdate & Date of observation yyyymmdd \\
\hline 15 & $124-133$ & A10 & $\ldots$ & FieldName & Name of RAVE field \\
\hline 16 & $135-135$ & I1 & $\ldots$ & PlateNumber & Plate number used \\
\hline 17 & $137-139$ & $\mathrm{I} 3$ & $\ldots$ & FiberNumber & Fiber number $[1,150]$ \\
\hline 18 & $141-144$ & I5 & $\mathrm{K}$ & Teff & Effective temperature \\
\hline 19 & $146-150$ & $\mathrm{~F} 4.2$ & $\operatorname{dex}$ & $\log g$ & Gravity \\
\hline 20 & $152-156$ & F5.2 & $\operatorname{dex}$ & Met & Uncalibrated $[\mathrm{M} / \mathrm{H}]$ \\
\hline 21 & $158-161$ & F4.2 & $\operatorname{dex}$ & alpha & {$[\alpha / \mathrm{Fe}]$} \\
\hline 22 & $163-167$ & F5.2 & $\operatorname{dex}$ & cMet & Calibrated $[\mathrm{M} / \mathrm{H}]$ \\
\hline 23 & $169-176$ & F8.1 & $\ldots$ & CHISQ & $\chi^{2}$ \\
\hline 24 & $178-182$ & F5.1 & $\ldots$ & $\mathrm{S} 2 \mathrm{~N}$ & Corrected signal to noise $\mathrm{S} 2 \mathrm{~N}$ \\
\hline 25 & $184-188$ & F5.1 & $\ldots$ & CorrelationCoeff & Tonry-Davis $R$ correlation coefficient \\
\hline 26 & $190-193$ & $\mathrm{~F} 4.2$ & $\ldots$ & PeakHeight & Height of correlation peak \\
\hline 27 & $195-200$ & F6.1 & $\mathrm{km} \mathrm{s}^{-1}$ & PeakWidth & Width of correlation peak \\
\hline 28 & $202-207$ & F6.1 & $\mathrm{km} \mathrm{s}^{-1}$ & CorrectionRV & Zero-point correction applied \\
\hline 29 & $209-214$ & F6.1 & $\mathrm{km} \mathrm{s}^{-1}$ & SkyRV & Measured HRV of sky \\
\hline 30 & $216-221$ & F6.1 & $\mathrm{km} \mathrm{s}^{-1}$ & SkyeRV & Error HRV of sky \\
\hline 31 & $223-227$ & F5.1 & $\ldots$ & SkyCorrelation & Sky Tonry-Davis correl. coefficient \\
\hline 32 & $229-233$ & F5.1 & $\ldots$ & SNRatio & Spectra $S / N$ \\
\hline 33 & $235-240$ & F6.3 & mag & BT & Tycho-2 BT magnitude \\
\hline 34 & $242-247$ & F6.3 & mag & eBT & Error BT \\
\hline 35 & $249-254$ & F6.3 & mag & VT & Tycho-2 VT magnitude \\
\hline 36 & $256-261$ & F6.3 & mag & eVT & Error VT \\
\hline 37 & $263-276$ & A12 & $\ldots$ & USNOID & USNO-B designation \\
\hline 38 & $278-283$ & F6.3 & mas & DisUSNO & Distance to USNO-B source \\
\hline 39 & $285-289$ & F5.2 & mag & B1 & USNO-B B1 magnitude \\
\hline 40 & $291-295$ & F5.2 & mag & $\mathrm{R} 1$ & USNO-B R1 magnitude \\
\hline 41 & $297-301$ & F5.2 & mag & B2 & USNO-B B2 magnitude \\
\hline 42 & $303-307$ & F5.2 & mag & $\mathrm{R} 2$ & USNO-B R2 magnitude \\
\hline 43 & $309-313$ & F5.2 & mag & IUSNO & USNO-B I magnitude \\
\hline 44 & $315-315$ & A1 & $\ldots$ & XidQualityUSNO & Cross-identification flag (2) \\
\hline 45 & $317-332$ & A16 & $\ldots$ & DENISID & DENIS designation \\
\hline 46 & $334-339$ & F6.3 & mas & DisDENIS & Distance to DENIS source \\
\hline 47 & $341-346$ & F6.3 & mag & IDENIS & DENIS I magnitude \\
\hline 48 & $348-351$ & $\mathrm{~F} 4.2$ & mag & eIDENIS & Error DENIS I magnitude \\
\hline 49 & $353-359$ & F6.3 & mag & JDENIS & DENIS J magnitude \\
\hline 50 & $360-363$ & F4.2 & mag & eJDENIS & Error DENIS J magnitude \\
\hline 51 & $365-370$ & F6.3 & mag & KDENIS & DENIS K magnitude \\
\hline 52 & $372-375$ & F4.2 & mag & eKDENIS & Error DENIS K magnitude \\
\hline 53 & $377-377$ & A1 & $\ldots$ & XidQualityDENIS & Cross-identification flag (2) \\
\hline 54 & $379-394$ & A16 & $\ldots$ & TWOMASSID & 2MASS designation \\
\hline 55 & $396-401$ & F6.3 & mas & Dis2MASS & Distance to 2MASS source \\
\hline 56 & $403-408$ & F6.3 & mag & J2MASS & 2MASS J magnitude \\
\hline 57 & $410-413$ & $\mathrm{~F} 4.2$ & mag & eJ2MASS & Error 2MASS J magnitude \\
\hline 58 & $415-420$ & F6.3 & mag & H2MASS & 2MASS H magnitude \\
\hline 59 & $422-425$ & F4.2 & mag & eH2MASS & Error 2MASS H magnitude \\
\hline 60 & $427-432$ & F6.3 & mag & K2MASS & 2MASS K magnitude \\
\hline 61 & $434-437$ & $\mathrm{~F} 4.2$ & mag & eK2MASS & error 2MASS K magnitude \\
\hline 62 & $439-441$ & A3 & $\ldots$ & TWOMASSphotFLAG & 2MASS photometric flag \\
\hline
\end{tabular}


Table 9

(Continued)

\begin{tabular}{lccccc}
\hline \hline $\begin{array}{l}\text { Column } \\
\text { number }\end{array}$ & $\begin{array}{c}\text { Character } \\
\text { range }\end{array}$ & Format & Units & Symbol & Description \\
\hline 63 & $443-443$ & A1 & $\ldots$ & XidQuality2MASS & Cross-identification flag (2) \\
64 & $445-447$ & A3 & $\ldots$ & ZeroPointFLAG & $\begin{array}{c}\text { Zero-point correction flag (3) } \\
65\end{array}$ \\
\hline $449-456$ & A8 & $\ldots$ & SpectraFLAG & Spectra quality flag (4) \\
\hline
\end{tabular}

Notes. (1) Flag value between 0 and 4: 0-no proper motion, 1-Tycho-2 proper motion, 2Supercosmos Sky Survey proper motion, 3-STARNET2.0 proper motion, 4-GSC1.2 $\times$ 2MASS proper motion, 5-UCAC-2 proper motions.

(2) Flag value is $A, B, C, D$, or $X: A-\operatorname{good}$ association, $B$-two solutions within 1 arcsec, $C$-more than two solutions within 1 arcsec, $D$-nearest neighbor more than 2 arcsec away, $X$-no possible counterpart found.

(3) Flag value of the form $F G H, F$ being for the entire plate, $G$ for the 50 fibers group to which the fiber belongs. If $H$ is set to *, the fiber is close to a 15-fiber gap. For $F$ and $G$ the values can be $A$, $B, C, D$, or $E$ : $A$-dispersion around correction lower than $1 \mathrm{~km} \mathrm{~s}^{-1}, B$-dispersion between 1 and $2 \mathrm{~km} \mathrm{~s}^{-1}, C$-dispersion between 2 and $3 \mathrm{~km} \mathrm{~s}^{-1}, D$-dispersion larger than $3 \mathrm{~km} \mathrm{~s}^{-1}, E$-less than 15 fibers available for the fit.

(4) Flag identifying possible problem in the spectra (values can be combined): a-asymmetric Ca lines, $\mathrm{c}$ - cosmic ray pollution, e-emission line spectra, $\mathrm{n}$-noise-dominated spectra, 1 -no lines visible, w-weak lines, $\mathrm{g}$ - strong ghost, $\mathrm{t}$ - bad template fit, $\mathrm{s}$ - strong residual sky emission, $\mathrm{cc}$ bad continuum, r-red part of the spectra shows problem, b-blue part of the spectra shows problem, $\mathrm{p}$ - possible binary/doubled lined, $\mathrm{x}$ - peculiar object.

temperature for the calibration of the metallicity. The final form of the calibration relation is

$$
[\mathrm{M} / \mathrm{H}]=0.938[\mathrm{~m} / \mathrm{H}]+0.767[\alpha / \mathrm{Fe}]-0.064 \log g+0.404
$$

where $[\mathrm{M} / \mathrm{H}]$ and $[\mathrm{m} / \mathrm{H}]$ denote the calibrated and the uncalibrated metallicities, respectively. This convention shall be used throughout the paper. Calibration nicely removes the trends mentioned before. Note that the gravity term nearly cancels the constant offset for main-sequence stars. Its inclusion in relation (20) is further justified by the fact that larger discrepancies in metallicity are constrained to lower gravities.

Inclusion of $\alpha$-enhancement $([\alpha / \mathrm{Fe}])$ in the calibration relation may seem a bit problematic. Its value is not known a priori, and we said in Section 3.3.5 that it cannot be accurately recovered by the RAVE pipeline (see the upper right panel of Figure 6). A typical recovery error of up to 0.15 dex makes $[\alpha / \mathrm{Fe}]$ values derived by RAVE hardly useful to decide if a certain star has an enhanced abundance of elements produced by capture of $\alpha$ particles or not. The reason is that the whole range of this parameter amounts to only 0.4 dex, i.e., not much larger than the recovery error. On the other hand, the $[\alpha / \mathrm{Fe}]$ values derived by RAVE are not random, so they statistically improve the accuracy of derived metallicity. A factor of 0.767 implies that they increase it by up to $0.3 \mathrm{dex}$ in extremely $\alpha$-enhanced stars. So, even though an accurate value of $[\alpha / \mathrm{Fe}]$ cannot be derived by RAVE, we know that its value is changing from star to star. In fact the enhancement of $\alpha$ elements is the first improvement on the abundance modeling of stars which reaches past the uniform scaling of solar abundances. RAVE stars are expected to show much of a variation in this parameter, as we are covering a wide range of stars from local dwarfs to the rather distant supergiants well above the Galactic plane. This is also the reason why we included the variation of $\alpha$-enhancement in the method to determine stellar parameters. If the value of $[\alpha / \mathrm{Fe}]$ were held fixed, or if it were calculated by some arbitrary relation, the resulting metallicity would be biased, with values shifted by up to 0.3 dex. We try to avoid such biases, so $[\alpha / \mathrm{Fe}]$ is part of the spectral processing, even though it cannot be accurately recovered.

The need for a metallicity calibration can also be partly due to our choice of the wavelength range. The largest contributors of strong absorption lines in RAVE spectra (for stars dominating the observed stellar population) are Ca II, Si I, Mg I, Ti I, and Fe I. All but the last one are produced by the capture of $\alpha$ particles. For the spectral type K0 III we have 54 prominent spectral lines of three $\alpha$-elements ( $\mathrm{Si} \mathrm{I}, \mathrm{Mg} \mathrm{I}$, and Ti I) and $60 \mathrm{Fe}$ I lines of similar strength. So $\alpha$-elements produce a similar number of lines as iron, not counting the very strong lines of $\alpha$-element $\mathrm{Ca}$ II which actually dominate any $\chi^{2}$ fit. So, when the RAVE pipeline tries to match the metallic content, the fits pointing to an enhanced $\alpha$ abundance or an increased metallicity are similar. As a result the pipeline may split the effect of metallicity into two parts, in the sense that it partly modifies the metallicity and partly adjusts the $\alpha$-enhancement. This may explain the large correlation between the $\alpha$-enhancement and metallicity, reflected in a large value of the coefficient $c_{1}$ in the calibration relation (Equation (20)). The ambiguity could be broken only by a higher $\mathrm{S} / \mathrm{N}$ spectrum covering a wider spectral range. This is also the reason why analysis methods involving equivalent widths of individual lines could not be used on a vast majority of RAVE spectra. A $\chi^{2}$ method described in Section 3.3 was chosen because it uses the whole spectrum and so makes the best use of the available information.

Figure 16 shows the situation after application of the calibration relation (20). All trends and offsets in the metallicity values have disappeared and the scatter between the derived and the reference metallicities is reduced from 0.37 to $0.18 \mathrm{dex}$ (Table 5).

We used Soubiran stars, APO observations, and M67 members to derive the calibration relation. The GCS stars can be used to check what we obtained. The GCS does not report metallicity $([\mathrm{M} / \mathrm{H}])$ but only iron abundance $([\mathrm{Fe} / \mathrm{H}])$. As mentioned before, the two are not identical. A substantial scatter in the metallicity versus iron abundance relation (as demonstrated in Figure 17 for the Soubiran stars) prevents us from 
Table 10

Results of the Re-Observation of 45 RAVE Stars at Apache Point Observatory

\begin{tabular}{|c|c|c|c|c|c|c|c|c|c|c|c|c|c|}
\hline \multirow[t]{2}{*}{ Name } & \multicolumn{4}{|c|}{ Echelle results } & \multicolumn{9}{|c|}{ RAVE values } \\
\hline & $T_{\text {eff }}$ & $\log g$ & {$[\mathrm{Fe} / \mathrm{H}]$} & {$[\mathrm{M} / \mathrm{H}]$} & Obsdate & FieldName & FibNum & $T_{\text {eff }}$ & $\log g$ & Met & $\alpha$ & CHISQ & $\mathrm{S} 2 \mathrm{~N}$ \\
\hline T4671_00811_1 & 5943 & 3.55 & -0.53 & -0.42 & 20040629 & $0030 \mathrm{~m} 06$ & 43 & 6006 & +3.89 & -0.66 & +0.17 & 935.21 & 44 \\
\hline T4926_00806_1 & 4633 & 2.89 & 0.25 & 0.25 & 20050322 & $1119 \mathrm{~m} 04$ & 110 & 4622 & +2.64 & -0.02 & +0.00 & 3253.97 & 60 \\
\hline T4927_01523_1 & 5500 & 4.23 & -0.19 & -0.15 & 20050321 & $1058 \mathrm{~m} 07$ & 97 & 5652 & +4.50 & -0.31 & +0.14 & 3163.09 & 71 \\
\hline T4931_00266_1 & 5235 & 2.44 & -1.24 & -1.02 & 20040629 & $1146 \mathrm{~m} 01$ & 45 & 5320 & +2.55 & -0.92 & +0.25 & 955.08 & 37 \\
\hline T5279_00819_1 & 4627 & 1.98 & -0.35 & -0.28 & 20041022 & $0136 \mathrm{~m} 15$ & 76 & 4642 & +2.02 & -0.67 & +0.16 & 5406.29 & 74 \\
\hline T5279_00819_1 & 4627 & 1.98 & -0.35 & -0.28 & 20041023 & $0136 \mathrm{~m} 15$ & 76 & 4568 & +1.96 & -0.68 & +0.21 & 970.44 & 63 \\
\hline T5279_01652_1 & 5565 & 2.80 & -0.05 & -0.04 & 20041022 & $0136 \mathrm{~m} 15$ & 108 & 5019 & +2.54 & -0.88 & +0.30 & 2348.39 & 65 \\
\hline T5279_01652_1 & 5565 & 2.80 & -0.05 & -0.04 & 20041023 & $0136 \mathrm{~m} 15$ & 108 & 5598 & +3.52 & -0.60 & +0.19 & 4490.77 & 75 \\
\hline T5310_00259_1 & 4370 & 0.63 & -1.47 & -1.25 & 20041202 & $0352 \mathrm{~m} 13$ & 28 & 4328 & +0.47 & -1.56 & +0.15 & 388.73 & 34 \\
\hline T5310_00788_1 & 4627 & 2.30 & -0.24 & -0.19 & 20041202 & $0352 \mathrm{~m} 13$ & 95 & 4548 & +1.93 & -0.45 & +0.03 & 319.58 & 32 \\
\hline T5491_01056_1 & 5986 & 3.67 & 0.16 & 0.16 & 20040510 & $1025 \mathrm{~m} 08$ & 141 & 6100 & +3.59 & -0.45 & +0.29 & 3596.59 & 69 \\
\hline T5496_00127_1 & 4594 & 2.12 & 0.00 & 0.00 & 20040502 & $1014 \mathrm{~m} 13$ & 88 & 4640 & +2.21 & -0.33 & +0.01 & 1748.35 & 62 \\
\hline T5499_00076_1 & 5944 & 3.90 & -0.79 & -0.62 & 20040531 & $1058 \mathrm{~m} 07$ & 38 & 6025 & +3.96 & -1.01 & +0.39 & 1574.16 & 53 \\
\hline T5507_01406_1 & 6075 & 3.69 & -0.84 & -0.66 & 20040530 & $1101 \mathrm{~m} 15$ & 89 & 6105 & +3.77 & -0.97 & +0.14 & 1742.15 & 81 \\
\hline T5543_00567_1 & 5497 & 4.27 & 0.21 & 0.21 & 20050330 & $1309 \mathrm{~m} 11$ & 146 & 5729 & +4.65 & +0.15 & +0.01 & 6685.34 & 94 \\
\hline T5562_00279_1 & 5090 & 3.24 & -0.06 & -0.05 & 20040607 & $1418 \mathrm{~m} 11$ & 77 & 5150 & +3.30 & -0.34 & +0.07 & 4356.54 & 76 \\
\hline T5762_00685_1 & 5191 & 3.08 & -0.86 & -0.67 & 20040629 & $2034 \mathrm{~m} 12$ & 40 & 5250 & +2.98 & -1.17 & +0.25 & 2550.69 & 70 \\
\hline T5789_00559_1 & 4501 & 1.41 & -1.06 & -0.84 & 20040627 & $2159 \mathrm{~m} 08$ & 47 & 4577 & +1.86 & -1.11 & +0.27 & 627.04 & 50 \\
\hline T5803_01091_1 & 5905 & 3.72 & -0.06 & -0.05 & 20040627 & $2159 \mathrm{~m} 08$ & 119 & 6011 & +3.76 & -0.31 & +0.26 & 2908.89 & 70 \\
\hline T5806_01423_1 & 5784 & 4.37 & 0.01 & 0.01 & 20040628 & $2216 \mathrm{~m} 13$ & 56 & 5945 & +4.41 & -0.34 & +0.16 & 1188.81 & 48 \\
\hline T5852_00128_1 & 4792 & 4.26 & -0.14 & -0.11 & 20041022 & $0136 \mathrm{~m} 15$ & 18 & 4922 & +4.57 & -0.19 & +0.00 & 4162.91 & 80 \\
\hline T5852_00128_1 & 4792 & 4.26 & -0.14 & -0.11 & 20041023 & $0136 \mathrm{~m} 15$ & 18 & 5204 & +4.88 & +0.19 & +0.00 & 443.61 & 35 \\
\hline T5852_00673_1 & 5412 & 2.95 & -0.72 & -0.57 & 20041022 & $0136 \mathrm{~m} 15$ & 2 & 5523 & +3.04 & -1.27 & +0.38 & 2772.21 & 65 \\
\hline T5852_00673_1 & 5412 & 2.95 & -0.72 & -0.57 & 20041023 & $0136 \mathrm{~m} 15$ & 2 & 5592 & +3.19 & -1.11 & +0.33 & 4107.41 & 67 \\
\hline T5852_01716_1 & 5574 & 3.59 & -1.19 & -0.97 & 20041022 & $0136 \mathrm{~m} 15$ & 34 & 5084 & +2.69 & -1.16 & +0.18 & 1567.50 & 51 \\
\hline T5852_01716_1 & 5574 & 3.59 & -1.19 & -0.97 & 20041023 & $0136 \mathrm{~m} 15$ & 34 & 5621 & +3.62 & -0.37 & +0.05 & 498.88 & 26 \\
\hline T6077_00047_1 & 5707 & 4.27 & 0.46 & 0.46 & 20050301 & $1101 \mathrm{~m} 15$ & 117 & 5775 & +4.27 & +0.18 & +0.03 & 3442.99 & 65 \\
\hline T6092_00615_1 & 5560 & 4.59 & 0.01 & 0.01 & 20050228 & $1144 \mathrm{~m} 20$ & 22 & 5496 & +4.68 & -0.11 & +0.00 & 2096.18 & 48 \\
\hline T6109_01354_1 & 6042 & 3.95 & 0.10 & 0.10 & 20050228 & $1232 \mathrm{~m} 22$ & 72 & 6083 & +4.24 & +0.05 & +0.02 & 2425.02 & 61 \\
\hline T6135_00087_1 & 4250 & 1.68 & -0.12 & -0.10 & 20050301 & $1345 \mathrm{~m} 21$ & 92 & 4295 & +1.69 & -0.47 & +0.07 & 2188.12 & 70 \\
\hline T6412_00004_1 & 5971 & 2.92 & -0.76 & -0.57 & 20040923 & $0014 \mathrm{~m} 21$ & 17 & 6041 & +3.22 & -1.00 & +0.19 & 571.02 & 57 \\
\hline T6412_00004_1 & 5971 & 2.92 & -0.76 & -0.57 & 20041024 & $0014 \mathrm{~m} 21$ & 19 & 6077 & +3.49 & -1.08 & +0.29 & 2600.99 & 93 \\
\hline T6459_00058_1 & 4526 & 1.92 & -0.34 & -0.27 & 20041230 & $0414 \mathrm{~m} 29$ & 66 & 4491 & +1.74 & -0.78 & +0.10 & 4411.66 & 74 \\
\hline T6473_00818_1 & 4594 & 2.42 & 0.07 & 0.07 & 20041023 & $0504 \mathrm{~m} 26$ & 84 & 4549 & +2.30 & -0.19 & +0.00 & 7898.21 & 84 \\
\hline T6478_00245_1 & 5503 & 3.54 & 0.07 & 0.07 & 20041023 & $0504 \mathrm{~m} 26$ & 106 & 5546 & +3.77 & -0.22 & +0.15 & 1435.45 & 51 \\
\hline T6484_00022_1 & 4599 & 2.01 & -0.36 & -0.28 & 20050128 & $0535 \mathrm{~m} 29$ & 73 & 4583 & +1.85 & -0.73 & +0.14 & 4421.43 & 82 \\
\hline T6705_00713_1 & 5813 & 4.57 & -0.02 & -0.02 & 20040528 & $1252 \mathrm{~m} 28$ & 84 & 5914 & +4.60 & -0.28 & +0.16 & 1167.70 & 66 \\
\hline T6705_00713_1 & 5813 & 4.57 & -0.02 & -0.02 & 20040529 & $1252 \mathrm{~m} 28$ & 84 & 5935 & +4.68 & -0.51 & +0.33 & 3947.94 & 86 \\
\hline T6904_00180_1 & 5889 & 3.21 & -0.96 & -0.75 & 20040607 & $2008 \mathrm{~m} 28$ & 42 & 5941 & +3.18 & -1.20 & +0.28 & 6137.75 & 95 \\
\hline T6975_00058_1 & 5294 & 2.96 & -0.79 & -0.62 & 20041024 & $2315 \mathrm{~m} 25$ & 16 & 5652 & +3.35 & -0.97 & +0.26 & 648.78 & 41 \\
\hline
\end{tabular}

Note. All stars passed visual inspection. Column names follow symbol names in Table 9, so Met marks the original (uncalibrated) value of the metallicity.

deriving a unique iron abundance to metallicity relation in the absence of additional information, as is the case with the GCS. In Figure 17, we therefore plot RAVE metallicity versus iron abundance from the GCS catalog. The uncalibrated RAVE metallicities (top panel) make the Soubiran and GCS occupy different regions of the metallicity/iron abundance diagram. But the calibrated RAVE metallicities (bottom panel) provide an excellent match. As said before, the GCS stars were not used in derivation of the calibration relation. The match is therefore further evidence that relation (20) can be trusted.

The calibrated metallicity can also be checked against predictions of semi-empirical models. Figure 18(a) plots the distribution of the calibrated metallicity determined from RAVE spectra, while 18(b) is an empirical prediction of the distribution of iron abundance. The latter was calculated using the Besançon Galactic model (Robin et al. 2003) with the apparent $I_{\text {DENIS }}$ magnitude distribution of RAVE stars and a random sample of objects more than $25^{\circ}$ from the Galactic plane, except for the inaccessible region $60^{\circ}<l<210^{\circ}$. The observed distribution in metallicity is more symmetric than its theoretical iron-abundance counterpart. The reason lies in the differences of the two quantities. Figure 17 shows that the metallicity is usually higher than iron abundance due to an enhanced presence of $\alpha$ elements. APO observations of RAVE stars 
Table 11

Results of the Re-Observation of 24 RAVE Stars with the Asiago Observatory Echelle Spectrograph

\begin{tabular}{|c|c|c|c|c|c|c|c|c|c|c|c|c|}
\hline \multirow[t]{2}{*}{ Name } & \multicolumn{3}{|c|}{ Echelle results } & \multicolumn{9}{|c|}{ RAVE values } \\
\hline & $T_{\text {eff }}$ & $\log g$ & {$[\mathrm{M} / \mathrm{H}]$} & Obsdate & FieldName & FibNum & $T_{\text {eff }}$ & $\log g$ & Met & $\alpha$ & $\mathrm{S} 2 \mathrm{~N}$ & SpectraFLAG \\
\hline T4678_00087_1 & 3938 & 3.00 & -1.03 & 20040629 & $0030 \mathrm{~m} 06$ & 38 & 3818 & 3.2 & -0.9 & 0.0 & 76 & $\mathrm{e}$ \\
\hline T4679_00388_1 & 6291 & 4.00 & -0.88 & 20040629 & $0030 \mathrm{~m} 06$ & 117 & 6102 & 3.8 & -0.7 & 0.2 & 73 & \\
\hline T4701_00802_1 & 4865 & 2.19 & -1.28 & 20041202 & $0238 \mathrm{~m} 05$ & 78 & 4652 & 2.2 & -1.1 & 0.3 & 94 & \\
\hline T4702_00944_1 & 5851 & 4.10 & -0.38 & 20041202 & $0238 \mathrm{~m} 05$ & 79 & 5704 & 4.7 & -0.4 & 0.2 & 80 & \\
\hline T4704_00341_1 & 5757 & 4.20 & -0.45 & 20041202 & $0238 \mathrm{~m} 05$ & 50 & 5667 & 4.0 & -0.6 & 0.2 & 76 & $\mathrm{p}$ \\
\hline T4749_00016_1 & 5178 & 3.06 & -0.65 & 20041202 & $0500 \mathrm{~m} 08$ & 64 & 4676 & 2.2 & -0.5 & 0.2 & 90 & \\
\hline T4749_00143_1 & 6928 & 3.97 & -0.30 & 20041202 & 0500m08 & 59 & 7034 & 4.1 & -0.2 & 0.1 & 73 & \\
\hline T4763_01210_1 & 4451 & 2.01 & -0.51 & 20041202 & $0500 \mathrm{~m} 08$ & 99 & 4301 & 1.6 & -0.6 & 0.1 & 72 & \\
\hline T5178_01006_1 & 4498 & 2.23 & -0.53 & 20040626 & $2054 \mathrm{~m} 02$ & 65 & 4682 & 2.1 & -0.4 & 0.0 & 68 & \\
\hline T5186_01028_1 & 4777 & 2.88 & 0.22 & 20040626 & $2054 \mathrm{~m} 02$ & 25 & 4628 & 2.5 & 0.1 & 0.0 & 62 & \\
\hline T5198_00021_1 & 4898 & 3.15 & 0.10 & 20040629 & $2119 \mathrm{~m} 03$ & 71 & 4653 & 2.9 & -0.0 & 0.0 & 77 & \\
\hline T5198_00784_1 & 7426 & 3.78 & -0.44 & 20040629 & $2119 \mathrm{~m} 03$ & 98 & 7375 & 4.0 & -0.3 & 0.0 & 83 & \\
\hline T5199_00143_1 & 7102 & 4.24 & -0.21 & 20040629 & $2119 \mathrm{~m} 03$ & 97 & 6986 & 4.0 & -0.1 & 0.1 & 99 & \\
\hline T5201_01410_1 & 4901 & 2.98 & 0.30 & 20040629 & $2119 \mathrm{~m} 03$ & 23 & 4641 & 2.7 & 0.2 & 0.0 & 68 & \\
\hline T5207_00294_1 & 4105 & 1.33 & -0.59 & 20040628 & $2133 \mathrm{~m} 08$ & 61 & 3999 & 1.0 & -0.7 & 0.2 & 80 & \\
\hline T5225_01299_1 & 4241 & 1.97 & -0.60 & 20040629 & $2212 \mathrm{~m} 04$ & 86 & 4104 & 1.0 & -0.7 & 0.1 & 80 & \\
\hline T5227_00846_1 & 5239 & 3.34 & -0.88 & 20040629 & $2212 \mathrm{~m} 04$ & 23 & 4856 & 3.1 & -0.6 & 0.2 & 60 & \\
\hline T5228_01074_1 & 5098 & 4.10 & -0.41 & 20040629 & $2212 \mathrm{~m} 04$ & 98 & 5219 & 4.7 & -0.3 & 0.0 & 72 & \\
\hline T5231_00546_1 & 7104 & 3.52 & -0.46 & 20040629 & $2212 \mathrm{~m} 04$ & 129 & 6891 & 3.8 & -0.7 & 0.3 & 85 & \\
\hline T5232_00783_1 & 4906 & 3.01 & -0.12 & 20040629 & $2212 \mathrm{~m} 04$ & 127 & 4760 & 2.8 & -0.3 & 0.0 & 72 & \\
\hline T5242_00324_1 & 3915 & 3.37 & -0.91 & 20040626 & $2313 \mathrm{~m} 03$ & 67 & 3530 & 4.0 & -0.7 & 0.0 & 73 & $\mathrm{e}$ \\
\hline T5244_00102_1 & 6523 & 3.28 & -0.54 & 20040626 & $2313 \mathrm{~m} 03$ & 96 & 6363 & 3.3 & -0.6 & 0.1 & 64 & \\
\hline T5246_00361_1 & 4890 & 2.44 & -0.89 & 20040626 & $2313 \mathrm{~m} 03$ & 81 & 4724 & 2.0 & -0.7 & 0.1 & 78 & \\
\hline T5323_01037_1 & 4880 & 2.48 & -0.66 & 20041202 & $0500 \mathrm{~m} 08$ & 139 & 4646 & 2.0 & -0.6 & 0.1 & 83 & \\
\hline
\end{tabular}

Note. Column names follow symbol names in Table 9, so Met marks the original (uncalibrated) values of the metallicity. Only stars with an empty SpectraFLAG were retained for further analysis.
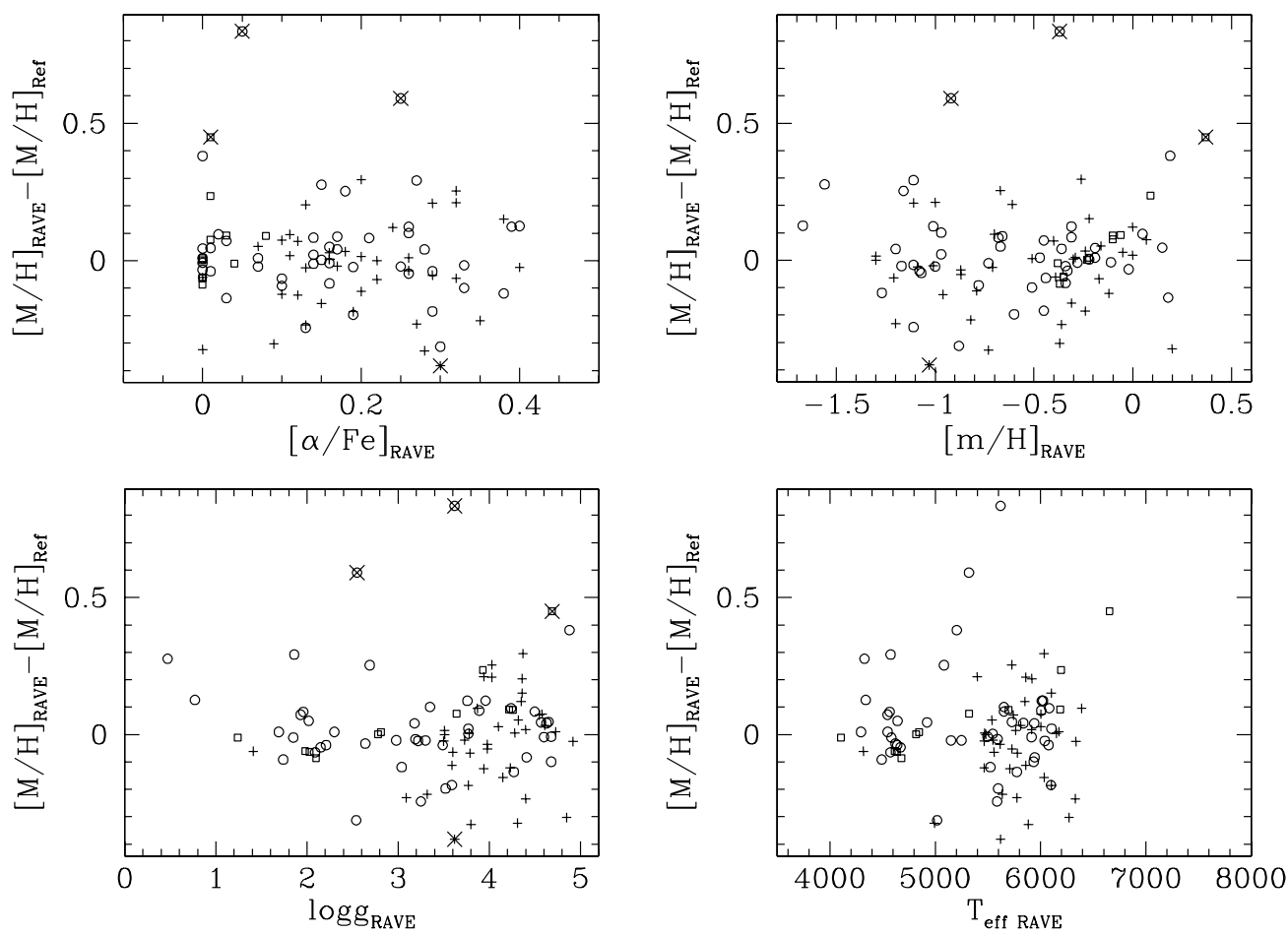

Figure 16. Comparison of calibrated metallicities derived by RAVE to the reference values. Symbol types are as in Figure 15. Points rejected during iterative calculation of the metallicity calibration are crossed out.

(Table 10) yield both iron abundance and metallicity, so they allow us to fit a statistical relation between metallicity and iron abundance

$$
[\mathrm{M} / \mathrm{H}]=[\mathrm{Fe} / \mathrm{H}]+0.11\left[1 \pm\left(1-e^{-3.6|[\mathrm{Fe} / \mathrm{H}]+0.55|}\right)\right],
$$

where the plus sign applies for $[\mathrm{Fe} / \mathrm{H}]<-0.55$ and the minus sign otherwise. The relation is plotted with a dashed line in Figure 17. It makes the metallicity 0.22 dex larger than the iron abundance for very metal-poor stars with $[\alpha / \mathrm{Fe}]=0.3$, while the difference vanishes when approaching the solar metallicity. 
Table 12

Results of the Observation of 49 Stars from the Soubiran \& Girard (2005) Catalog

\begin{tabular}{|c|c|c|c|c|c|c|c|c|c|c|c|c|}
\hline \multirow[t]{2}{*}{ Name } & \multicolumn{4}{|c|}{ Echelle results } & \multicolumn{8}{|c|}{ RAVE values } \\
\hline & $T_{\text {eff }}$ & $\log g$ & {$[\mathrm{Fe} / \mathrm{H}]$} & {$[\mathrm{M} / \mathrm{H}]$} & Obsdate & FieldName & FibNum & $T_{\text {eff }}$ & $\log g$ & Met & $\alpha$ & $\mathrm{S} 2 \mathrm{~N}$ \\
\hline 13420 & & 41 & -1.04 & -0.90 & & & 114 & 5765 & & & & 90 \\
\hline HD136351 & 341 & 4.04 & 0.01 & 0.00 & 0070422 & & 114 & 5151 & & & & 235 \\
\hline HD157467 & 016 & 3.72 & 0.11 & 0.27 & 0070422 & $1726 \mathrm{~m} 03$ & 33 & 103 & +3.77 & -0.24 & +0.19 & 212 \\
\hline HD156635 & 136 & 4.28 & -0.10 & 0.12 & 0070422 & & 41 & 330 & +4.40 & & & 243 \\
\hline HD15 & 687 & 4.38 & 0.00 & -0.01 & $00^{\prime}$ & 1 & 67 & 813 & +4.61 & -0.24 & .18 & 206 \\
\hline HD158 & 164 & .80 & -0.77 & -0.49 & & & 109 & 727 & +4.03 & & & 147 \\
\hline HD15 & 227 & 3.94 & -0.71 & -0.51 & 20 & & 114 & 5391 & & & & 206 \\
\hline HD1 & 540 & 4.49 & -1.17 & -0.87 & 23 & & 120 & 481 & & & & 144 \\
\hline HD149612 & 5680 & 4.53 & -0.48 & -0.43 & 23 & 165 & 18 & 5615 & +3.98 & 87 & 26 & 208 \\
\hline HD153075 & 5770 & 4.17 & -0.57 & -0.39 & 423 & & 114 & 728 & +3.98 & & & 214 \\
\hline HD13 & 001 & 4.09 & 0.13 & 0.06 & 5 & & 120 & 02 & +4.36 & -0 & & 165 \\
\hline HD172051 & 52 & 4.49 & -0.29 & -0.24 & 25 & & 120 & 42 & +4.55 & & & 257 \\
\hline HD1 & 53 & 4.00 & 0.24 & 0.24 & & & 116 & 5781 & & & & 202 \\
\hline HD1 & 05 & 4.48 & -0.63 & -0.53 & 06 & & 144 & 09 & & & & 171 \\
\hline HD1 & 856 & 4.12 & 0.28 & 0.18 & & & 120 & 05 & & & & 238 \\
\hline & & 4.31 & & & & & & & & & & 172 \\
\hline 1 & & 4.30 & 0.32 & .19 & 6 & & 20 & & & & & 246 \\
\hline HD1 & 632 & 4.01 & -0.67 & -0.51 & 07 & & 20 & 66 & & & & 131 \\
\hline HD121004 & 5635 & 4.39 & -0.73 & -0.55 & 07 & & 116 & 5918 & .36 & -0 . & & 94 \\
\hline HD1 & 320 & 3.91 & 0.23 & 19 & 07 & & 120 & 04 & .10 & -0.05 & 16 & 226 \\
\hline HD1C & 617 & 4.30 & -0.27 & -0.23 & & & & 68 & & & & 196 \\
\hline & & 4.31 & & & & & & & & & & 196 \\
\hline HD1 & & & 0.22 & & & & & & & & & 147 \\
\hline HD1 & 200 & 3.98 & -0.56 & -0.48 & & & 120 & 775 & & & & 263 \\
\hline HD10 & 5978 & 3.75 & -0.25 & -0.09 & 09 & & 70 & 5638 & +3.32 & -0.82 & 35 & 117 \\
\hline HD104304 & 5361 & 4.47 & 0.14 & 0.22 & 20070509 & 1159 & 116 & 5466 & +4.23 & -0.12 & +0.10 & 164 \\
\hline HD163799 & 5764 & 4.02 & -0.92 & -0.65 & 20070509 & $1758 \mathrm{~m} 22$ & 116 & 5559 & +3.60 & -1.21 & +0.32 & 160 \\
\hline HD091345 & & 4.43 & & & & & & & & & & 150 \\
\hline HD102365 & & 4.55 & & & & & & & & & & 37 \\
\hline HD120559 & & 4.48 & & & & & & & & & & 61 \\
\hline HD134088 & 5625 & 4.37 & -0.87 & -0.63 & 20070505 & $1508 \mathrm{~m} 08$ & 121 & 5547 & +3.73 & -1.01 & +0.17 & 144 \\
\hline HD152449 & 6096 & 4.18 & -0.05 & 0.12 & 20070505 & $1647 \mathrm{~m} 02$ & 96 & 6034 & +4.15 & -0.31 & +0.15 & 239 \\
\hline HD152986 & 6074 & 4.25 & -0.17 & 0.02 & 20070505 & $1647 \mathrm{~m} 02$ & 108 & 5885 & +3.80 & -0.73 & +0.28 & 160 \\
\hline HD162396 & 6079 & 4.15 & -0.37 & -0.30 & 20070505 & $1752 \mathrm{~m} 42$ & 120 & 5859 & +3.59 & -0.79 & +0.20 & 319 \\
\hline HD177565 & 5625 & 4.21 & 0.03 & -0.02 & & & & 5539 & +4.32 & -0.16 & +0.07 & 155 \\
\hline HD106516 & 6208 & 4.39 & -0.71 & -0.45 & & & 120 & 6337 & +4.92 & & & 44 \\
\hline HD125072 & & 4.62 & 0.49 & & & & 116 & 4992 & & & +0.00 & 32 \\
\hline HD145937 & 5813 & 4.07 & -0.60 & -0.18 & 20070522 & $1610 \mathrm{~m} 06$ & 120 & 5621 & +3.62 & -1.03 & +0.30 & 196 \\
\hline
\end{tabular}

Note. All stars passed visual inspection. Column names follow symbol names in Table 9, so Met marks the original (uncalibrated) value of the metallicity.

The relation is very similar to that of Salaris et al. (1993). If this relation, together with metallicity errors typical for the RAVE observations (Equation (22) and Figure 19), is used, the resulting histograms (Figure 18(c)) are very similar to the observed ones (Figure 18(a)). Peaks of the histograms match to within $0.06 \mathrm{dex}$, while the width is $\sim 25 \%$ larger in the model compared to the observations. A somewhat larger width of the model histograms suggests that the error estimates for the RAVE metallicity are conservative. Note however that the Besançon model predicts a smaller fraction of low gravity stars $(\log g \leqslant 3.0)$ than observed.

The description of stellar chemical composition by metallicity and $\alpha$-enhancement values is a simplification. Generally, the individual stellar elemental abundances (including those of the $\alpha$-elements) do not scale linearly or in a constant ratio with those of the Sun, and spectral lines of some elements are not present in the RAVE wavelength range. Individual element abundances frequently scatter by 0.2 or 0.3 dex if compared to the iron abundance (Soubiran \& Girard 2005). This fact of nature is also the cause of a large scatter of metallicity versus iron abundance in the Soubiran sample, depicted by gray points in Figure 17. The metallicity change of $0.2-0.3 \mathrm{dex}$, as introduced by the calibration relation, is therefore comparable to the intrinsic scatter of individual element abundances in stars. So it would be very difficult to provide a detailed physical explanation for the calibration relation between the metallicities derived by equivalent width or photometry methods and those obtained by a $\chi^{2}$ analysis. Equation (21) therefore reflects only approximate general trends. Nevertheless, it allows us to check that the distribution of the calibrated metallicities derived by RAVE is consistent with the predictions of the Besançon Galactic model.

\subsubsection{Method for Stellar Parameter Error Estimation}

Errors associated with a given stellar parameter depend on the $\mathrm{S} / \mathrm{N}$ of the spectrum and on the spectral properties of the star. We discuss them in turn. The calibration data have very different values of $\mathrm{S} / \mathrm{N}$, in general higher than typical RAVE 
Table 13

Results of the Observation of 12 Members of the Open Cluster M67

\begin{tabular}{lccccccccrr}
\hline \hline Name & Adopted & \multicolumn{10}{c}{ RAVE values } \\
\cline { 3 - 10 } & {$[\mathrm{M} / \mathrm{H}]$} & Obsdate & FieldName & FibNum & $T_{\text {eff }}$ & $\log g$ & Met & $\alpha$ & S2N \\
\hline M67-6469 & 0.01 & 20070409 & $0851 \mathrm{p} 11$ & 42 & 4318 & +1.41 & -0.39 & +0.00 & 138 \\
M67-0084 & 0.01 & 20070409 & $0851 \mathrm{p} 11$ & 72 & 4614 & +1.98 & -0.35 & +0.00 & 86 \\
M67-6495 & 0.01 & 20070409 & $0851 \mathrm{p} 11$ & 76 & 4105 & +1.24 & -0.38 & +0.04 & 117 \\
M67-0223 & 0.01 & 20070409 & $0851 \mathrm{p} 11$ & 91 & 4634 & +2.03 & -0.35 & +0.00 & 73 \\
M67-0218 & 0.01 & 20070409 & $0851 \mathrm{p} 11$ & 105 & 4820 & +2.78 & -0.23 & +0.00 & 75 \\
M67-0286 & 0.01 & 20070409 & $0851 \mathrm{p} 11$ & 120 & 4678 & +2.10 & -0.37 & +0.00 & 102 \\
M67-0135 & 0.01 & 20070409 & $0851 \mathrm{p} 11$ & 146 & 4847 & +2.81 & -0.22 & +0.00 & 64 \\
M67-0115 & 0.01 & 20070601 & $0851 \mathrm{p} 11$ & 13 & 6189 & +4.22 & -0.06 & +0.03 & 25 \\
M67-0046 & 0.01 & 20070601 & $0851 \mathrm{p} 11$ & 58 & 5697 & +4.26 & -0.10 & +0.08 & 22 \\
M67-7859 & 0.01 & 20070601 & $0851 \mathrm{p} 11$ & 72 & 6656 & +4.69 & +0.37 & +0.01 & 18 \\
M67-0192 & 0.01 & 20070601 & $0851 \mathrm{p} 11$ & 101 & 6194 & +3.93 & +0.09 & +0.01 & 24 \\
M67-0227 & 0.01 & 20070601 & $0851 \mathrm{p} 11$ & 143 & 5320 & +3.64 & -0.10 & +0.01 & 24 \\
\hline
\end{tabular}

Note. $[\mathrm{M} / \mathrm{H}]$ marks the adopted value of metallicity from the literature, while other values, including the uncalibrated metallicity (Met) were obtained from RAVE observations. Column names follow symbol names in Table 9. The M67 numbering system is summarized in http://www.univie.ac.at/webda//cgi-bin/ocl_page.cgi?cluster=m67. Stars with numbers between 0001 and 0295 were numbered by Fagerholm (1906). Stars with 64xx numbers are from Montgomery et al. (1993) and star 7859 is from Fan et al. (1996).
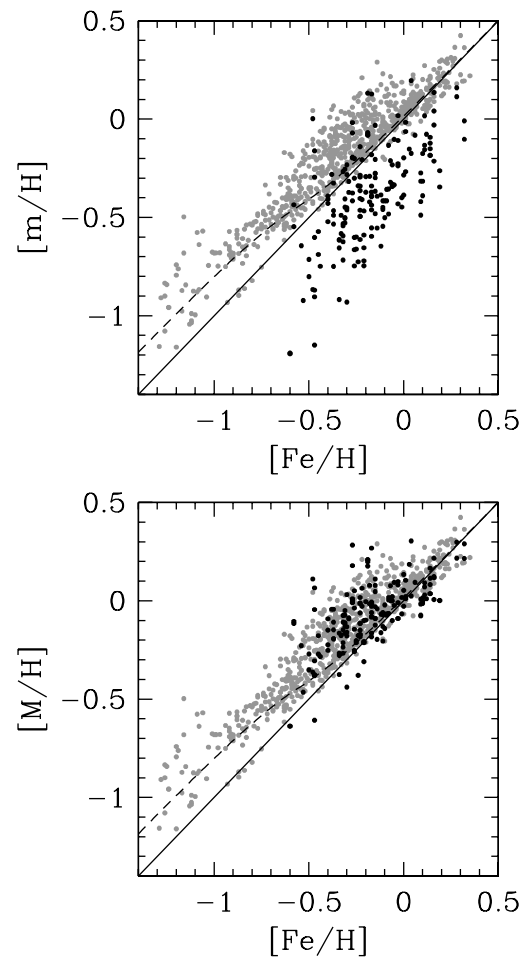

Figure 17. Relation between iron abundance and metallicity. The gray points mark the positions of all stars in the Soubiran \& Girard (2005) catalog. The black ones are RAVE observations of stars from the GCS, with the uncalibrated values of metallicity in the top graph and the calibrated ones in the bottom one. The solid line traces the 1:1 relation, while the dashed one is the mean relation between the iron abundance and the metallicity derived from the APO observations (Equation (21)).

survey data. The average $\mathrm{S} / \mathrm{N}$ for the survey stars for which we publish values of stellar parameters is 41 . So we choose $\mathrm{S} / \mathrm{N}=40$ as the reference value. The error estimate $\sigma_{40}$ below therefore refers to a star with $\mathrm{S} / \mathrm{N}=40$. Extensive Monte Carlo simulations show that the error $\sigma$ for a stellar parameter has the following scaling with the $\mathrm{S} / \mathrm{N}$ of the observed spectrum:
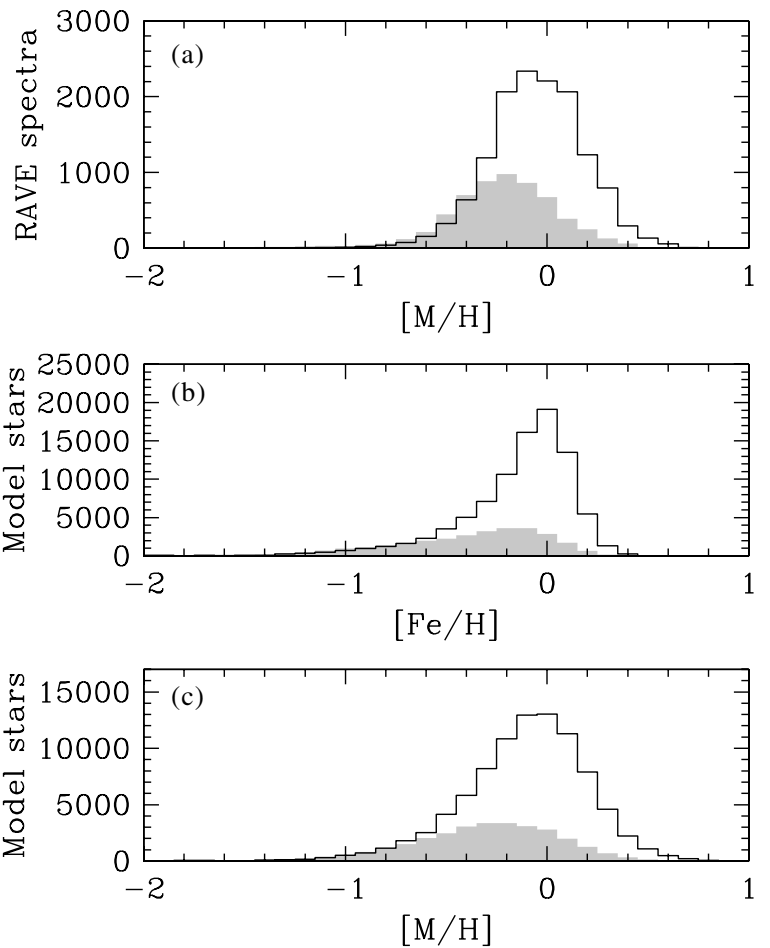

Figure 18. Comparison of the metallicity of the observed targets to the iron abundances from the Besançon model. The clear and the shaded histograms mark high gravity $(\log g>3.0)$ and low gravity $(\log g \leqslant 3.0)$ objects. Panel (a) plots all RAVE spectra with $|b| \geqslant 25^{\circ}$. Panel (b) is a distribution of stars drawn at random from the Besançon model. The stars are more than $25^{\circ}$ from the plane and have the same distribution of $I$ apparent magnitudes as $I_{\text {DENIS magnitudes }}$ in RAVE. Panel (c) is a histogram from the same Besançon model, using the iron abundance to metallicity relation from Equation (21) and convolved with typical RAVE observational errors.

$$
\sigma=r^{k} \sigma_{40}
$$

where

$$
r= \begin{cases}(\mathrm{S} / \mathrm{N}) / 40, & \text { if } \mathrm{S} / \mathrm{N}<80 \\ 80 / 40, & \text { otherwise }\end{cases}
$$


and the coefficient $k$ has the value of -0.848 for temperature, -0.733 for gravity, and -0.703 for metallicity. The simulations used 63 high S/N spectra observed by RAVE for which high-resolution echelle spectroscopy has also been obtained in Asiago or at the APO. We assumed that the analysis of echelle spectroscopy yields the true values of the parameters for these stars and studied how the values derived by the RAVE pipeline would worsen if additional Gaussian noise were added to the RAVE spectra. We found that the offsets in mean values of stellar parameters appear only at $\mathrm{S} / \mathrm{N}<6$ (an offset in temperature at $\mathrm{S} / \mathrm{N}=6$ is $100 \mathrm{~K}$ ) and disappear at higher $\mathrm{S} / \mathrm{Ns}$. Gaussian noise is not the only source of the problems with weak signal spectra. Systematic effects due to scattered light, fiber crosstalk, and incomplete removal of flat-field interference patterns prevent a reliable parameter determination in a large fraction of spectra with $\mathrm{S} / \mathrm{N}<13$. So we decided to publish radial velocities down to $\mathrm{S} / \mathrm{N}=6$, while stellar parameter values are published only for spectra with $\mathrm{S} / \mathrm{N}>13$. The latter decision influences $\sim 13 \%$ of RAVE spectra which have $6<\mathrm{S} / \mathrm{N}<13$.

Simulations also show that the errors on the parameters do not continue to improve for stars with $\mathrm{S} / \mathrm{N}>80$, because systematic errors tend to dominate over statistical noise in such low-noise cases. So we flatten out the error decrease for $\mathrm{S} / \mathrm{N}>80$ in Equation (23).

The choice of the reference $\mathrm{S} / \mathrm{N}$ of 40 means that the errors discussed below should be about twice as large for the noisiest spectra with published parameters, and about twice as small for spectra with the largest value of $\mathrm{S} / \mathrm{N}$.

The calibration datasets (Table 4) contain only stars hotter than $4000 \mathrm{~K}$ and cooler than $7500 \mathrm{~K}$. The majority of these stars are on or close to the main sequence with a metallicity similar to the solar value. Many of the RAVE program stars are of this type, but not all. For example, one cannot judge the errors of hot stars or very metal-poor stars from these datasets. So we need to use simulations to estimate the value of $\sigma_{40}$ in Equation (22), i.e., how the error depends on the type of star that is observed. Relative errors are estimated from a theoretical grid of Kurucz models, but the observed calibration datasets are used for the scaling of the relative to absolute error values and for verifying the results.

We start with a theoretical normalized spectrum from the precomputed Kurucz grid and investigate the increase of the rms when we compare it with grid-point spectra in its vicinity in the five-dimensional space of $T_{\text {eff }}, \log g,[\mathrm{M} / \mathrm{H}],[\alpha / \mathrm{Fe}]$, and $V_{\text {rot }}$. If we denote the values of five parameters for the initial spectrum as $P_{i}(i=1, \ldots, 5)$, and if $p_{i}(i=1, \ldots, 5)$ denote their values at a grid point in its vicinity, the estimate $\sigma_{j}$ of an error of parameter $j$ for the initial spectrum can be obtained from the minimum of $\mathrm{rms}_{j}=\min \left\{\operatorname{rms}\left(p_{1}, p_{2}, \ldots, p_{5}\right), p_{j} \neq P_{j}\right\}$. We assume that an increase of rms has a similar effect on the parameter estimation as an increase of a noise level. So $\mathrm{rms}_{j}$ is inversely proportional to the $\mathrm{S} / \mathrm{N}$ of the normalized spectrum, but the dependence of the error $\sigma_{j}$ of the parameter $j$ on the $\mathrm{S} / \mathrm{N}$ is given by the value of the coefficient $k$ in Equation (22). The only remaining factor is the proportionality constant. It is derived by the assumption that $68.2 \%$ of all calibration spectra should have the value of the parameter $j$ determined by the RAVE pipeline within $\pm \sigma_{j}$ of the reference value.

The scheme allows us to estimate errors in all corners of the parameter space covered by Kurucz models, i.e., even in parts where we lack any calibration spectra. Calibration spectra are used exclusively for scaling of the $\sigma_{40}$ value of a given stellar parameter in Equation (22). This scaling was done

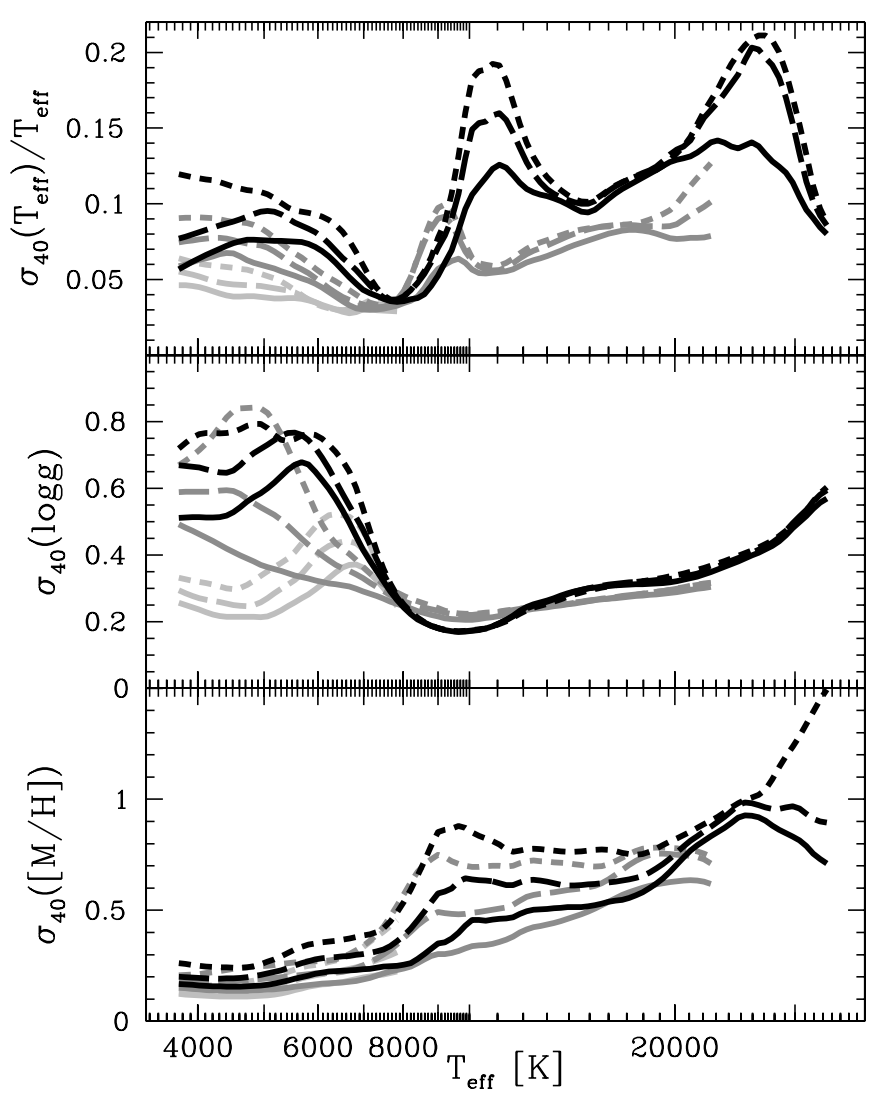

Figure 19. Errors for temperature, gravity and metallicity as a function of temperature. The nine curves are errors for three values of gravity and three values of metallicity: black curves are errors for MS stars $(\log g=4.5)$, gray for subgiants $(\log g=3.0)$, and light-gray curves for supergiants $(\log g=1.0)$. Solid lines are for solar metallicity, long dashed ones are for $[\mathrm{M} / \mathrm{H}]=-0.5$ and short dashed ones for $[\mathrm{M} / \mathrm{H}]=-1.0$. All errors apply for a star with $\mathrm{S} / \mathrm{N}=40$, the ones for other noise levels follow from Equation (22).

assuming that $\sim 2 / 3$ of RAVE calibration objects should have a given parameter within one standard deviation of the true value obtained from high-resolution observations. So we can check if the relative number of calibration objects within, e.g., 0.5 or 2 standard deviations conforms to the normal distribution. A positive answer would support the results. Next, we discuss the accuracy of each stellar parameter in turn.

\subsubsection{Temperature Accuracy}

The top panel of Figure 19 plots the standard deviation of temperature as a function of temperature for stars with $\mathrm{S} / \mathrm{N}=40$. The value of the standard deviation is divided by temperature. So an ordinate value of 0.05 at $6000 \mathrm{~K}$ denotes a standard deviation of $300 \mathrm{~K}$. The nine curves are errors for three values of gravity and three values of metallicity. Light gray curves are for supergiants ( $\log g=1.0$ ), gray ones for subgiants $(\log g=3.0$ ), and black ones for MS stars $(\log g=4.5)$. Solid lines are for solar metallicity, while long dashed ones are for $[\mathrm{M} / \mathrm{H}]=-0.5$ and short dashed ones for $[\mathrm{M} / \mathrm{H}]=-1.0$.

Typical errors for stars cooler than $9000 \mathrm{~K}$ are around $400 \mathrm{~K}$. The errors are the smallest for supergiants. Their atmospheres are the most transparent ones, so that a wealth of spectral lines arising at different optical depths can improve the temperature accuracy. Understandably the errors for metal poor stars are larger than for their solar counterparts. The errors get considerably worse for hot stars $(T>9000 \mathrm{~K})$, where most metal lines are missing and the spectrum is largely dominated 
by hydrogen lines. All these trends can be seen from Figure 5 where wavelength intervals affected by temperature change are marked by red lines.

These error estimates are rather conservative because we assumed that any discrepancy arises only because of RAVE errors, i.e., that the calibration datasets are error free. As mentioned already in the discussion on zero-point offsets (Section 4.2.2), this is not always the case. In particular, the errors in temperature would be $20 \%$ smaller if we did not use the GCS stars in error estimation.

Figure 20(a) plots the cumulative distribution of errors for the calibration stars used to derive the temperature errors. Line types and grayscale tones are the same as in Figure 19. We see that $68 \%$ of our stars have their error within $1 \sigma$, a condition we used for scaling. But the distribution of stars along the error curve also closely follows the normal distribution. This supports the error estimates given in Figure 19.

\subsubsection{Gravity Accuracy}

The middle panel of Figure 19 plots errors in gravity as a function of temperature. Strong wings of hydrogen lines which are sensitive to gravity allow small gravity errors in hot stars (see blue marks in Figure 5 which mark gravity-sensitive regions). On the other hand rather narrow metallic lines in the RAVE wavelength range, including those of $\mathrm{Ca}$ II, do not allow an accurate determination of gravity in cool stars. The gravity error in cool stars has a strong gravity dependence: in dwarfs it is large, but the rather transparent atmospheres of giant stars still allow for a reasonably accurate gravity determination. In any case the errors in gravity do not exceed 0.8 dex, which still allows the determination of a luminosity class.

Figure 20(b) is similar to Figure 20(a) in the sense that it plots the errors of calibration stars. Again, we have $68 \%$ of the stars with errors smaller than the standard deviation, a condition used to calibrate the errors in Figure 19. Departures from the normal distribution of errors can be explained by a rather small number of spectra used to determine the gravity errors.

\subsubsection{Metallicity Accuracy}

The bottom panel of Figure 19 plots standard deviations of the calibrated metallicity $([\mathrm{M} / \mathrm{H}])$. The typical error for stars cooler than $7000 \mathrm{~K}$ is 0.2 dex. The error for hotter stars is understandably much larger, as these stars lack most of the metallic lines in their spectra (lack of green marks in hot spectra in Figure 5). Figure 20(c) shows that the distribution of errors is very close to the normal one.

\subsubsection{Errors on Other Parameters}

The rotational velocity will be a topic of a separate paper which will discuss fast rotating stars, so we do not estimate its error here. The $\alpha$-enhancement value is part of this data release, but given the fact that the Kurucz grid covers only two values $(0.0$ and 0.4$)$ it is very hard to estimate its error. We note that our metallicity has a typical error of $0.2 \mathrm{dex}$, so it seems likely that the statistical error on $\alpha$-enhancement is larger. Note that this is comparable to the value of $[\alpha / \mathrm{Fe}]=0.22$ reached in typical metal-poor stars. So although the $[\alpha / \mathrm{Fe}]$ parameter is useful to improve the accuracy of derived metallicities (Equation (20)), its value is not accurate enough to be trusted for individual stars.

\subsection{Detection of Peculiar and Problematic Spectra}

Errors on temperature, gravity, and metallicity have been presented for a range of normal stars. We estimate that these
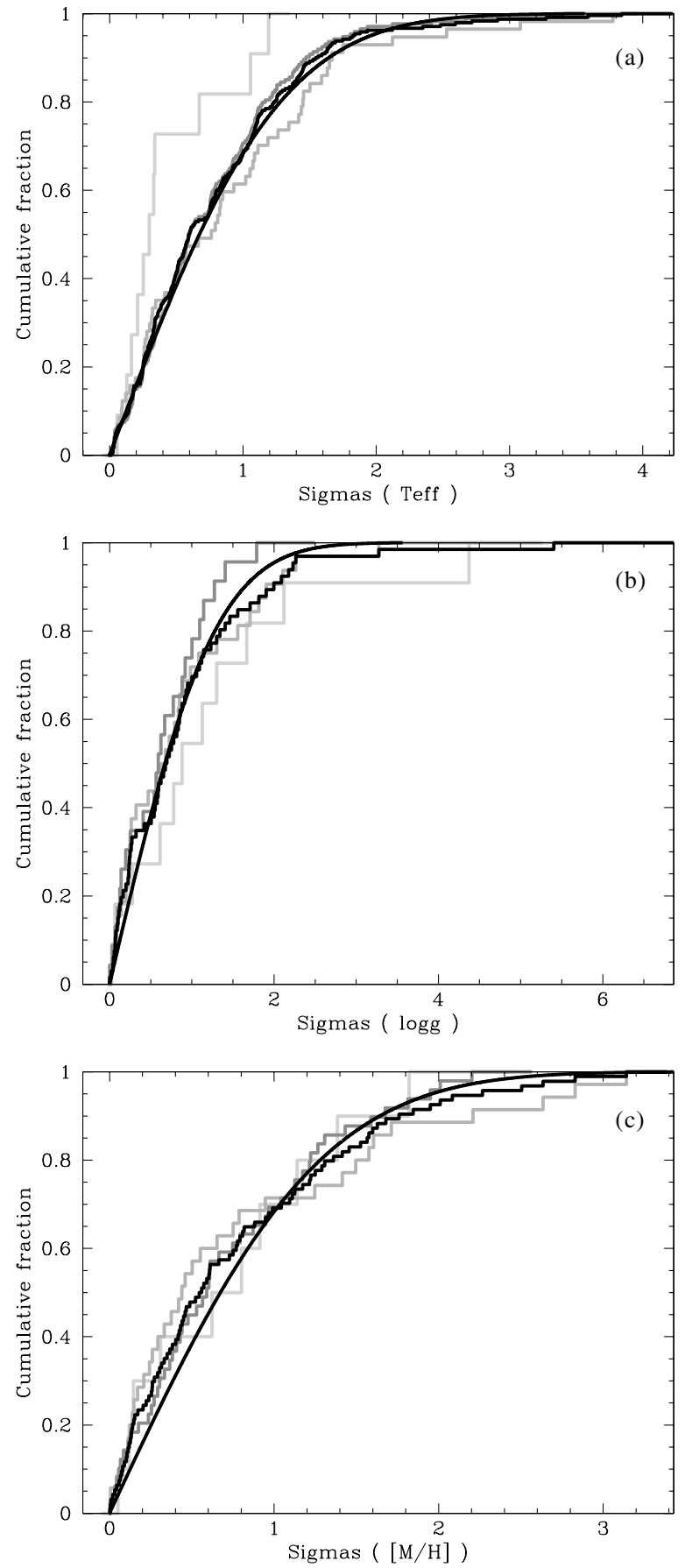

Figure 20. Cumulative fraction of reference spectra with the difference between the RAVE and the reference value within the given number of standard deviations: (a) for temperature, (b) for gravity, (c) for metallicity. The black histogram is for all calibration stars, while the gray ones denote different gravity ranges: dark gray marks $\log g \geqslant 3.75$, middle gray is for $2.0 \leqslant \log g<3.75$, and light gray corresponds to $\log g<2.0$. The smooth curve plots an ideal normal distribution.

errors are statistically accurate to $\sim 30 \%$. Errors for other normal stars could be derived by linear interpolation. But not all stars have normal spectra. RAVE observed a number of binaries, emission-type objects, and other peculiar stars, while occasionally a spectrum of a normal star is jeopardized by systematic errors. So it is vital to identify such objects.

RV information is present in all spectral lines. Still, a very noisy spectrum, too uncertain a wavelength solution, or other systematic errors could lead to unreliable results. The 


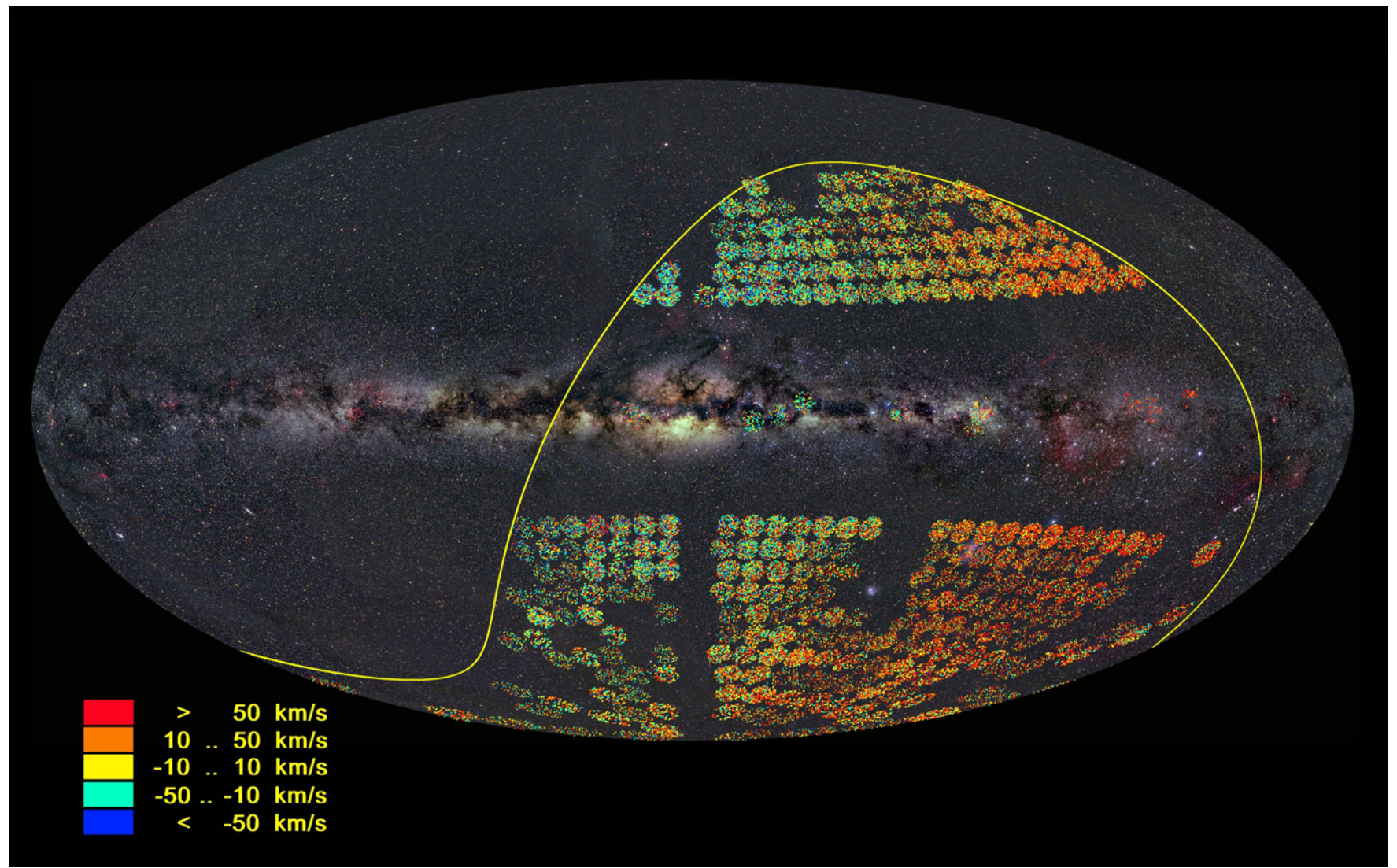

Figure 21. Aitoff projection in Galactic coordinates of RAVE second data release fields. The yellow line represents the celestial equator and the background is from Axel Mellinger's all-sky panorama.

simulations showed that the RV is not systematically affected by noise if the $\mathrm{S} / \mathrm{N}$ is larger than 6 . At lower $\mathrm{S} / \mathrm{Ns}$, the best template identified by our matching method would be systematically offset (for $100 \mathrm{~K}$ or more in temperature) therefore affecting the $\mathrm{RV}$ accuracy. This effect is not present at higher S/Ns. So we calculated the $\mathrm{S} / \mathrm{N}$ for each spectrum and visually checked if the calcium lines (and at higher S/Ns, also others) show a mutually consistent RV. 698 spectra were rejected, mostly because their $\mathrm{S} / \mathrm{N}<6$, and are not part of this data release.

The measurement of stellar parameters requires a higher $\mathrm{S} / \mathrm{N}$. We adopted a limit of $\mathrm{S} / \mathrm{N}=13$ as the minimum value. Note that this limit is still quite conservative as it corresponds to $\sim 8 \%$ error in the flux of each pixel. So the published values of stellar parameters are statistically correct, but parameters for individual stars with $\mathrm{S} / \mathrm{N}<20$ should be considered as preliminary. This data release contains 3411 such relatively noisy spectra.

All spectra new to this release were visually checked. The goal is to avoid systematic errors, as well as to identify types of objects which are not properly covered by our grid of theoretical models. In the latter case, large and arbitrary errors in values of stellar parameters could result. Such objects include double-lined spectroscopic binary stars, emission-type objects, and other peculiar stars. We do not publish values of stellar parameters for such objects, but only the values of their RV which is calculated in the same way as for normal stars. So we are consistent with the first data release. We also avoid arbitrary decisions in cases of undetected or marginally detected binaries. Their published RV is somewhere between the instantaneous velocities of the two components and does not correspond necessarily to the barycentric one. The physical analysis of detected double-lined spectroscopic binaries will be presented in a separate paper. But Seabroke et al. (2008) showed that they do not affect statistical kinematic Galactic studies significantly.

The first data release contained 26,079 spectra for which we published radial velocities but no stellar parameters. Also in the data new to this data release, there are 3343 spectra without published stellar parameters. From these there are 140 emissiontype spectra, 135 double-lined binary spectra and 86 spectra of peculiar stars. Other spectra without published parameters have $\mathrm{S} / \mathrm{N}<13$ or are affected by systematic problems. Table 6 summarizes the results. The last column quotes the number of different objects with a given classification. Some stars occasionally show normal spectra and we publish the values of their stellar parameters, but in other occasions they show some kind of peculiarity or systematic problem, so that their parameter values are not published. So the first number in the last column is not an exact sum of the two numbers below it.

\subsection{Repeated Observations}

Most stars are observed by RAVE only once, but some observations are repeated for calibration purposes. 1893 objects in the present data release have more than one spectrum. Table 6 explains that the present release contains 51,829 spectra of 49,327 different stars. Note that the latter number is smaller than the number of stars of individual types. This is a consequence of the fact that a spectrum of a star may appear as a double lined binary star in one spectrum, and as an entirely normal single star in another one (taken close to conjunction). So the star would be counted as a member of two types. A definite classification of all stars in this data release is beyond 
the scope of this paper. We plan to pursue follow-up studies for particular types of objects, like spectroscopic binaries, and present them in separate papers.

Repeated observations allow a comparison of the measured properties of these stars. If we assume that values for a given star do not change with time, the scatter can be used to estimate errors on RV and the values of the stellar parameters. This assumption may not always be true, for example in the case of binaries or intrinsically variable stars. So we assumed that the sigma of a parameter is the value which comprises $68.2 \%$ of the differences between the measured values of a parameter and its average value for a given star. This way we minimize the effect of large deviations of (rare) variable objects and measure an effective standard deviation of a given parameter.

The data release contains 1893 objects with two or more measurements of RV. The dispersion of measurements for a particular object is smaller than $1.80 \mathrm{~km} \mathrm{~s}^{-1}$ in $68.2 \%$ of the cases and smaller than $7.9 \mathrm{~km} \mathrm{~s}^{-1}$ in $95 \%$ of the cases.

For 855 objects, we also have two or more spectra with published stellar parameters. In this case, the dispersion of velocities is within $1.66 \mathrm{~km} \mathrm{~s}^{-1}$ (68.2\% of objects) and $6.1 \mathrm{~km} \mathrm{~s}^{-1}$ ( $95 \%$ of cases). The corresponding scatter in the temperature is $135 \mathrm{~K}(68.2 \%)$ and $414 \mathrm{~K}(95 \%)$, for gravity 0.2 dex $(68.2 \%)$ and 0.5 dex $(95 \%)$, and for the calibrated metallicity 0.1 dex $(68.2 \%)$ and 0.2 dex (95\%).

Spectra of repeated objects share the same distribution of the $\mathrm{S} / \mathrm{N}$ as all RAVE stars. Their typical $\mathrm{S} / \mathrm{N}$ of 40 is smaller than for the reference datasets (see Figure 12); still the above-quoted value for the dispersion of radial velocities is similar to the errors of the reference datasets (Table 3). Also the dispersions of stellar parameter values as derived from the repeated observations are smaller than the dispersions for the reference datasets (Table 5). One expects a higher internal consistency of the repeated observations, as these are free from zero-point errors. But the zero-point errors are very small for both RV and stellar parameters (Tables 3 and 5). Note that our error estimates of $\mathrm{RV}$ and stellar parameter values are derived assuming that the reference values from the external datasets are error free, and this may not always be the case. We conclude that the error estimates on RV (Section 4.1) and stellar parameters (Section 4.2) are quite conservative.

\section{SECOND DATA RELEASE}

\subsection{Global Properties}

The second public data release of the RAVE data (RAVE DR2) is accessible online. It can be queried or retrieved from the Vizier database at the Centre de Donnees Astronomiques de Strasbourg (CDS), as well as from the RAVE collaboration Web site (www.rave-survey.org). Table 9 describes its column entries. The tools to query and extract information are described in Paper I.

The result of the RAVE survey are radial velocities and values of stellar parameters (temperature, gravity, and metallicity). Metallicity is given twice: as coming from the data reduction pipeline $([\mathrm{m} / \mathrm{H}])$ and after application of calibration Equation (20) $([\mathrm{M} / \mathrm{H}]$, see Section 4.2 .3 for details). The latter also includes the value of $\alpha$-enhancement. So the catalog includes also the estimated values of $[\alpha / \mathrm{Fe}]$. As explained in Section 4.2.8 this is provided mainly for calibration purposes and is not intended to infer properties of individual objects.

Figure 21 plots the general pattern of (heliocentric) radial velocities. The dipole distribution is due to solar motion with
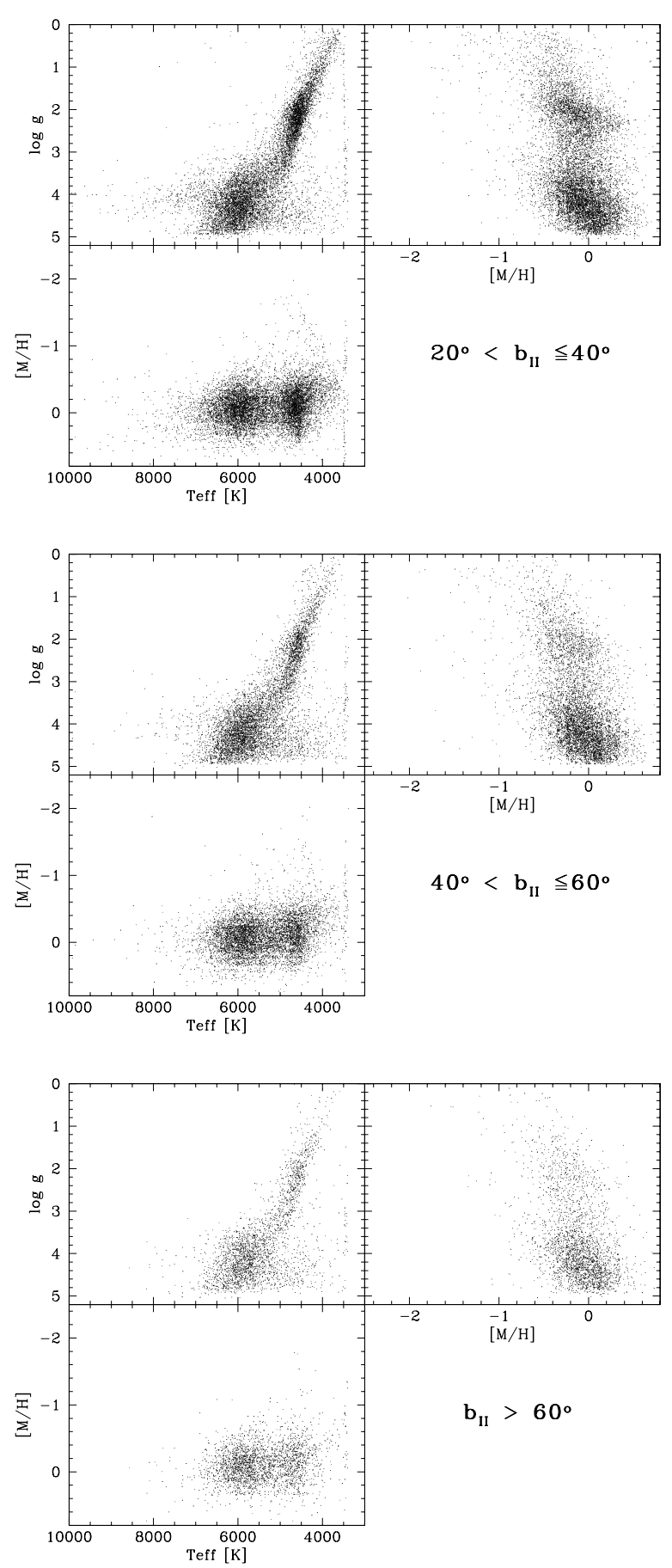

Figure 22. Temperature-gravity-metallicity wedge for three bands in Galactic latitude. Spectra with $T_{\text {eff }} \sim 3500 \mathrm{~K}$ are at the edge of the grid of spectral templates: so their temperatures should be used with caution, usually as an upper limit to the real value.

respect to the Local Standard of Rest (LSR). Spatial coverage away from the Galactic plane is rather good, with the exception of stars at small Galactic longitudes. These areas have already been observed and will be part of the next data release.

The investigation of properties of the stellar parameters and their links to Galactic dynamics and formation history are beyond the scope of this paper. To illustrate the situation, we outline just two plots. Figure 22 shows the location of all spectra on the temperature-gravity-metallicity wedge. Note the main sequence and giant groups, their relative frequency and metallicity 

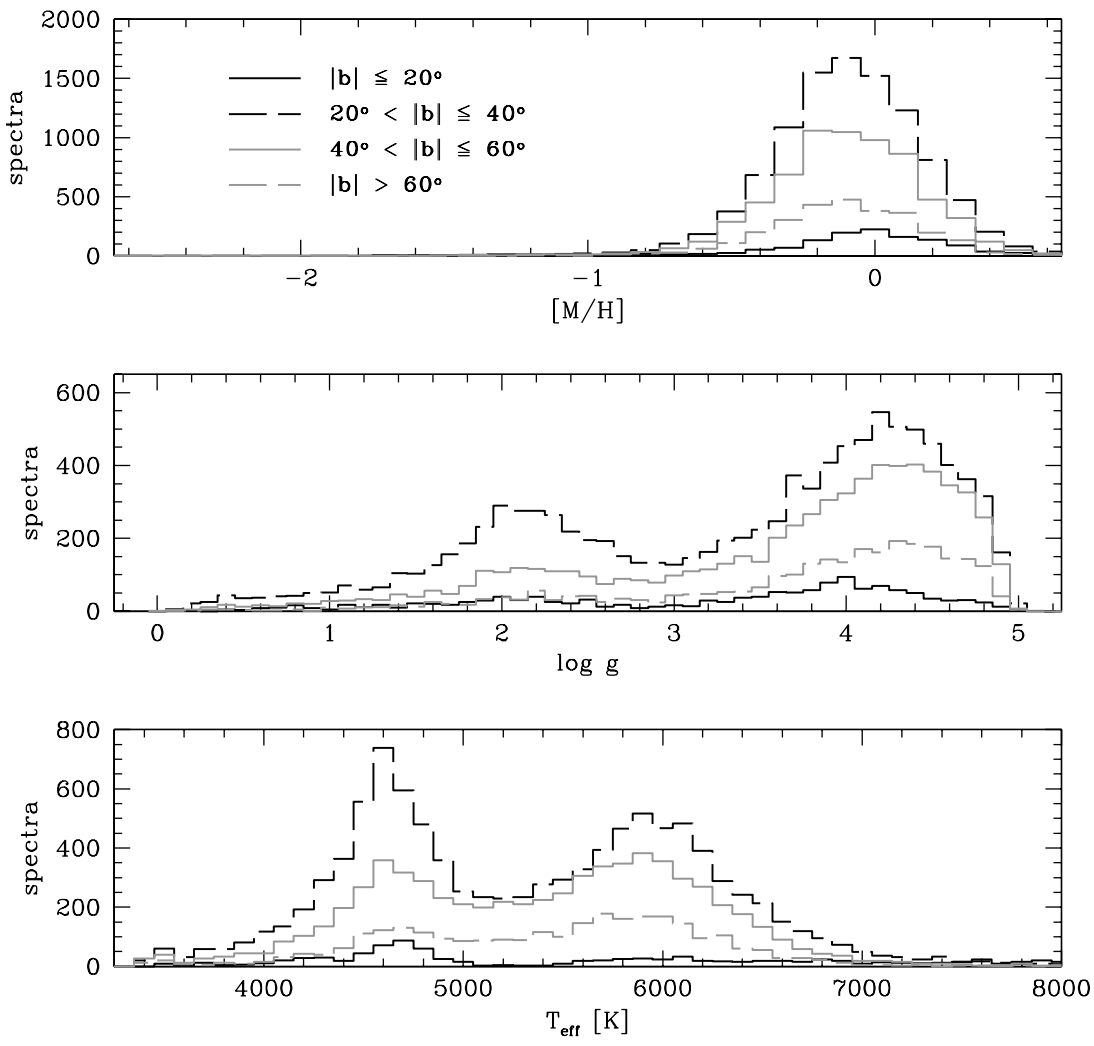

Figure 23. Temperature, gravity, and metallicity histograms for spectra with published stellar parameters. Histograms for individual Galactic latitude bands are plotted separately with the key given in the top panel. Spectra with $|b| \leqslant 20^{\circ}$ are calibration fields. Note the increasing fraction of MS stars at high Galactic latitudes.

distribution for three bands in Galactic latitude. Figure 23 plots histograms of the parameters, again for different bands in Galactic latitude. The fraction of MS stars increases with the distance from the Galactic plane. This can be understood by the fact that the RAVE targets have rather similar apparent magnitudes (Figure 1). Giants therefore trace a more distant population, and those at high latitudes would already be members of the (scarcely populated) Galactic halo.

\subsection{Photometry}

The data release includes cross-identification with optical and near-IR catalogs (USNO-B, DENIS, 2MASS), where the nearest-neighbor criterion was used for matching. Similar to the first data release, we provide the distance to the nearest neighbor and a quality flag on the reliability of the match. Note that this is important as RAVE uses optical fibers with a projected diameter of 6.7 arcsec on the sky. Table 7 shows that nearly all stars were successfully matched for the 2MASS and USNO-B catalogs, while only about $3 / 4$ of the stars lie in the sky area covered by the DENIS catalog. For the matched stars we include USNO-B B1, R1, B2, R2, and $I$ magnitudes, DENIS $I$, $J$, and $K$ magnitudes, and 2MASS $J, H$, and $K$ magnitudes. As mentioned, our wavelength range is best represented by the $I$ filter. With the publication of the second release of the DENIS catalog, we decided to use the DENIS I magnitude as our reference in planning of future observations.

We note here that the DENIS I magnitudes appear to be affected by saturation for stars with $I<10$. Following a comment from a member of the DENIS team, we compared the DENIS and 2MASS magnitude scales. 2MASS does not provide an I magnitude. However, the transformation

$$
I_{2 \mathrm{MASS}}=J_{2 \mathrm{MASS}}+1.103(J-K)_{2 \mathrm{MASS}}+0.07
$$

gives an approximate $I$ magnitude on the DENIS system from the 2MASS $J K$ photometry for giants and dwarfs with $(J-K)<0.65$. First, we confirmed that the $(J-K)_{2 \mathrm{MASS}}$ colors are consistent with the temperature derived by RAVE for all objects. We then compared the DENIS and 2MASS I magnitudes for all stars in the current data release having errors $<0.05$ in both of these $I$ magnitudes. For most stars with $I_{\text {DENIS }}>10$, the magnitudes agree within the expected errors. However we note that (1) the relation between the two magnitudes becomes nonlinear for the $\sim 16 \%$ of the brightest stars with $I_{\text {DENIS }}<10$, and (2) about $8 \%$ of the fainter stars with apparently well-determined magnitudes from both catalogs have differences $\left|\left(I_{\text {DENIS }}-I_{2 \mathrm{MASS}}\right)\right|>0.2$. Some stars have differences greater than \pm 3 mag. We therefore propose to avoid using $I_{\text {DENIS }}$ magnitudes when the condition $-0.2<\left(I_{\text {DENIS }}-J_{2 \text { MASS }}\right)-\left(J_{2 \text { MASS }}-K_{2 \text { MASS }}\right)<0.6$ is not met. Figure 4 follows this advice and avoids the scatter due to some problematic $I_{\text {DENIS }}$ magnitude values.

\subsection{Proper Motions}

Similar to the first data release, the proper motions are taken from Starnet 2.0 and Tycho-2 catalogs (see Paper I for a complete discussion). These values are however not available for $\sim 30 \%$ of the spectra and in Paper I we bridged the gap with proper motions from the SSS catalog. The SSS catalog suffers from substantial uncertainties, so we now attempted a cross-identification with the UCAC2 catalog (Zacharias et al. 2004). RAVE coordinates were used to search for the nearest two neighbors in the UCAC 2 catalog. It turned out that it suffices to use the data for the first next neighbor, as there were no cases where the matching distance to the first neighbor was less than $3 \operatorname{arcsec}$ while that to the second one was less than $6 \operatorname{arcsec}$. The 
Table 14

Results of Observations of GCS stars

\begin{tabular}{|c|c|c|c|c|c|c|c|c|c|c|c|c|c|c|c|}
\hline \multirow[t]{2}{*}{ Name } & \multicolumn{4}{|c|}{ GCS } & \multicolumn{11}{|c|}{ RAVE values } \\
\hline & $\log \left(T_{\text {eff }}\right)$ & {$[\mathrm{Fe} / \mathrm{H}]$} & HRV & eHRV & Obsdate & FieldName & FibNum & HRV & eHRV & $T_{\text {eff }}$ & $\log g$ & Met & $\alpha$ & $\mathrm{S} 2 \mathrm{~N}$ & SpectraFLAG \\
\hline HD 13386 & 3.708 & 0.28 & 31.9 & 0.2 & 20050827 & $0220 \mathrm{~m} 29 \mathrm{~b}$ & 021 & 36.758 & 1.440 & 5445 & +4.6 & +0.2 & +0.0 & 38 & \\
\hline HD 14294 & 3.796 & -0.30 & -11.0 & 0.2 & 20050827 & $0220 \mathrm{~m} 29 \mathrm{~b}$ & 066 & -8.309 & 1.087 & 6213 & +4.1 & -0.7 & +0.2 & 35 & $\mathrm{t}$ \\
\hline HD 14555 & 3.720 & -0.27 & 0.9 & 0.4 & 20050827 & $0220 \mathrm{~m} 29 \mathrm{~b}$ & 079 & 1.942 & 1.483 & 5463 & +4.7 & -0.2 & +0.1 & 37 & \\
\hline HD 15337 & 3.707 & 0.14 & -4.6 & 0.3 & 20050827 & $0220 \mathrm{~m} 29 \mathrm{~b}$ & 093 & -5.358 & 1.033 & 5244 & +4.3 & -0.1 & +0.0 & 42 & \\
\hline HD 14868 & 3.760 & -0.17 & 28.8 & 0.3 & 20050827 & $0220 m 29 b$ & 117 & 29.347 & 0.684 & 5892 & +4.5 & -0.4 & +0.3 & 85 & \\
\hline HD 14680 & 3.699 & -0.03 & 51.5 & 0.2 & 20050827 & $0220 \mathrm{~m} 29 \mathrm{~b}$ & 149 & 56.242 & 2.313 & 5582 & +4.6 & +0.1 & +0.0 & 18 & $\mathrm{cc}$ \\
\hline HD 13386 & 3.708 & 0.28 & 31.9 & 0.2 & 20050827 & $0220 \mathrm{~m} 29$ & 021 & 32.172 & 1.009 & 5376 & +4.4 & +0.1 & +0.0 & 111 & \\
\hline HD 14294 & 3.796 & -0.30 & -11.0 & 0.2 & 20050827 & $0220 \mathrm{~m} 29$ & 066 & -10.571 & 1.028 & 5952 & +3.6 & -0.9 & +0.3 & 97 & \\
\hline HD 14555 & 3.720 & -0.27 & 0.9 & 0.4 & 20050827 & $0220 \mathrm{~m} 29$ & 079 & 1.308 & 1.259 & 5608 & +4.7 & -0.1 & +0.0 & 95 & \\
\hline HD 15337 & 3.707 & 0.14 & -4.6 & 0.3 & 20050827 & $0220 \mathrm{~m} 29$ & 093 & -4.636 & 0.960 & 5255 & +4.3 & -0.1 & +0.0 & 98 & \\
\hline HD 14868 & 3.760 & -0.17 & 28.8 & 0.3 & 20050827 & $0220 \mathrm{~m} 29$ & 117 & 29.436 & 0.665 & 5972 & +4.6 & -0.3 & +0.2 & 159 & \\
\hline HD 14680 & 3.699 & -0.03 & 51.5 & 0.2 & 20050827 & $0220 \mathrm{~m} 29$ & 149 & 51.758 & 0.759 & 5066 & +4.8 & -0.2 & +0.0 & 89 & \\
\hline HD 21216 & 3.797 & -0.11 & 12.3 & 1.1 & 20050827 & $0328 \mathrm{~m} 06 \mathrm{~b}$ & 043 & 18.183 & 1.412 & 6320 & +4.6 & -0.5 & +0.2 & 57 & \\
\hline HD 21977 & 3.764 & 0.11 & 26.6 & 0.2 & 20050827 & $0328 \mathrm{~m} 06 \mathrm{~b}$ & 085 & 28.603 & 1.371 & 5977 & +4.4 & -0.1 & +0.0 & 32 & \\
\hline HD 21543 & 3.749 & -0.60 & 63.7 & 0.1 & 20050827 & $0328 \mathrm{~m} 06 \mathrm{~b}$ & 119 & 62.293 & 0.896 & 5004 & +3.5 & -1.2 & +0.4 & 38 & \\
\hline HD 21995 & 3.767 & -0.21 & -16.2 & 0.2 & 20050827 & $0328 \mathrm{~m} 06 \mathrm{~b}$ & 143 & -17.914 & 1.011 & 5807 & +4.2 & -0.7 & +0.4 & 56 & \\
\hline HD 21216 & 3.797 & -0.11 & 12.3 & 1.1 & 20050827 & $0328 \mathrm{~m} 06$ & 043 & 16.003 & 1.220 & 6494 & +4.9 & -0.4 & +0.2 & 146 & \\
\hline HD 21977 & 3.764 & 0.11 & 26.6 & 0.2 & 20050827 & $0328 \mathrm{~m} 06$ & 085 & 27.628 & 1.082 & 6062 & +4.7 & -0.2 & +0.2 & 75 & \\
\hline HD 21543 & 3.749 & -0.60 & 63.7 & 0.1 & 20050827 & $0328 \mathrm{~m} 06$ & 119 & 64.017 & 0.977 & 5344 & +3.5 & -1.2 & +0.4 & 71 & \\
\hline HD 21995 & 3.767 & -0.21 & -16.2 & 0.2 & 20050827 & $0328 \mathrm{~m} 06$ & 143 & -15.020 & 0.761 & 5676 & +4.1 & -0.7 & +0.4 & 119 & \\
\hline HD 150875 & 3.814 & -0.32 & -16.5 & 0.3 & 20050827 & $1652 \mathrm{~m} 03 \mathrm{~b}$ & 047 & -13.025 & 0.993 & 6491 & +3.7 & -0.5 & +0.2 & 79 & \\
\hline HD 151258 & 3.781 & -0.65 & -16.6 & 0.3 & 20050827 & $1652 \mathrm{~m} 03 \mathrm{~b}$ & 064 & -13.372 & 1.011 & 5972 & +4.1 & -0.9 & +0.2 & 50 & $\mathrm{cc}$ \\
\hline HD 152986 & 3.783 & -0.23 & -8.0 & 0.3 & 20050827 & $1652 \mathrm{~m} 03 \mathrm{~b}$ & 093 & -5.371 & 1.214 & 5953 & +4.0 & -0.4 & +0.1 & 78 & \\
\hline HD 153479 & 3.797 & -0.25 & 24.5 & 0.4 & 20050827 & $1652 \mathrm{~m} 03 \mathrm{~b}$ & 124 & 25.621 & 0.909 & 6124 & +4.3 & -0.6 & +0.3 & 38 & \\
\hline HD 153240 & 3.788 & -0.15 & -22.9 & 0.2 & 20050827 & $1652 \mathrm{~m} 03 \mathrm{~b}$ & 132 & -29.001 & 0.929 & 6472 & +4.8 & -0.3 & +0.2 & 69 & \\
\hline HD 150875 & 3.814 & -0.32 & -16.5 & 0.3 & 20050827 & $1652 \mathrm{~m} 03$ & 047 & -14.034 & 0.744 & 6423 & +3.6 & -0.6 & +0.2 & 150 & \\
\hline HD 151258 & 3.781 & -0.65 & -16.6 & 0.3 & 20050827 & $1652 \mathrm{~m} 03$ & 064 & -14.952 & 0.711 & 5884 & +3.6 & -0.8 & +0.0 & 107 & $\mathrm{t}$ \\
\hline HD 152986 & 3.783 & -0.23 & -8.0 & 0.3 & 20050827 & $1652 \mathrm{~m} 03$ & 093 & -6.667 & 1.054 & 6168 & +4.1 & -0.3 & +0.1 & 130 & \\
\hline HD 153479 & 3.797 & -0.25 & 24.5 & 0.4 & 20050827 & $1652 \mathrm{~m} 03$ & 124 & 25.676 & 0.795 & 6150 & +4.2 & -0.7 & +0.4 & 106 & \\
\hline HD 153240 & 3.788 & -0.15 & -22.9 & 0.2 & 20050827 & $1652 \mathrm{~m} 03$ & 132 & -29.131 & 0.758 & 6374 & +4.8 & -0.4 & +0.2 & 143 & \\
\hline HD 181177 & 3.754 & -0.23 & 48.3 & 0.2 & 20050827 & 1930m62b & 004 & 51.749 & 1.155 & 5890 & +4.5 & -0.3 & +0.2 & 63 & \\
\hline HD 181249 & 3.697 & 0.12 & -42.9 & 0.3 & 20050827 & $1930 \mathrm{~m} 62 \mathrm{~b}$ & 013 & -42.012 & 2.068 & 5098 & +4.2 & +0.0 & +0.0 & 17 & $\mathrm{cc}$ \\
\hline HD 180120 & 3.815 & -0.23 & -0.7 & 0.8 & 20050827 & $1930 \mathrm{~m} 62 \mathrm{~b}$ & 022 & 0.740 & 0.983 & 6339 & +4.0 & -0.6 & +0.3 & 47 & \\
\hline HD 177104 & 3.811 & -0.32 & -17.0 & 0.7 & 20050827 & $1930 \mathrm{~m} 62 \mathrm{~b}$ & 035 & -16.017 & 0.687 & 6329 & +4.2 & -0.7 & +0.4 & 67 & \\
\hline HD 185260 & 3.734 & 0.04 & -34.7 & 0.2 & 20050827 & $1930 \mathrm{~m} 62 \mathrm{~b}$ & 091 & -30.514 & 1.883 & 5717 & +4.5 & +0.2 & +0.0 & 17 & \\
\hline HD 186784 & 3.794 & -0.01 & -0.5 & 0.2 & 20050827 & $1930 \mathrm{~m} 62 \mathrm{~b}$ & 104 & 2.611 & 1.150 & 6133 & +3.8 & -0.4 & +0.3 & 45 & \\
\hline HD 185579 & 3.806 & -0.03 & -2.0 & 0.6 & 20050827 & $1930 \mathrm{~m} 62 \mathrm{~b}$ & 110 & 0.087 & 1.688 & 6515 & +4.8 & -0.1 & +0.1 & 32 & \\
\hline HD 185142 & 3.810 & -0.17 & 17.5 & 0.4 & 20050827 & $1930 \mathrm{~m} 62 \mathrm{~b}$ & 120 & 21.527 & 0.947 & 6095 & +3.9 & -0.5 & +0.3 & 66 & \\
\hline HD 171278 & 3.818 & -0.26 & -16.8 & 0.4 & 20050827 & $1930 \mathrm{~m} 62 \mathrm{~b}$ & 149 & 569.046 & 28.089 & 1629 & +0.1 & +0.1 & +0.1 & 000 & $\mathrm{n}$ \\
\hline HD 181177 & 3.754 & -0.23 & 48.3 & 0.2 & 20050827 & $1930 \mathrm{~m} 62$ & 004 & 48.909 & 0.785 & 5924 & +4.3 & -0.4 & +0.3 & 133 & \\
\hline HD 181249 & 3.697 & 0.12 & -42.9 & 0.3 & 20050827 & 1930m62 & 013 & -42.520 & 1.161 & 5156 & +4.7 & -0.1 & +0.0 & 50 & \\
\hline HD 180120 & 3.815 & -0.23 & -0.7 & 0.8 & 20050827 & $1930 \mathrm{~m} 62$ & 022 & 2.021 & 0.701 & 6419 & +4.1 & -0.5 & +0.2 & 121 & \\
\hline HD 177104 & 3.811 & -0.32 & -17.0 & 0.7 & 20050827 & 1930m62 & 035 & -15.111 & 0.661 & 6559 & +4.3 & -0.5 & +0.3 & 136 & \\
\hline HD 185260 & 3.734 & 0.04 & -34.7 & 0.2 & 20050827 & $1930 \mathrm{~m} 62$ & 091 & -33.909 & 1.314 & 5617 & +4.2 & -0.2 & +0.1 & 65 & \\
\hline HD 186784 & 3.794 & -0.01 & -0.5 & 0.2 & 20050827 & $1930 \mathrm{~m} 62$ & 104 & -0.487 & 0.643 & 6127 & +3.9 & -0.4 & +0.2 & 100 & \\
\hline HD 185579 & 3.806 & -0.03 & -2.0 & 0.6 & 20050827 & $1930 \mathrm{~m} 62$ & 110 & -2.211 & 0.866 & 6303 & +4.2 & -0.4 & +0.3 & 106 & \\
\hline HD 185142 & 3.810 & -0.17 & 17.5 & 0.4 & 20050827 & $1930 \mathrm{~m} 62$ & 120 & 19.737 & 0.900 & 6101 & +3.7 & -0.6 & +0.3 & 157 & \\
\hline HD 171278 & 3.818 & -0.26 & -16.8 & 0.4 & 20050827 & $1930 \mathrm{~m} 62$ & 149 & 464.747 & 9.245 & 2787 & +0.5 & +0.4 & +0.3 & 000 & $\mathrm{n}$ \\
\hline HD 192628 & 3.763 & -0.30 & -53.7 & 0.2 & 20050827 & $2017 \mathrm{~m} 15 \mathrm{~b}$ & 007 & 48.773 & 1.593 & 5697 & +4.0 & -0.3 & +0.0 & 26 & \\
\hline HD 192266 & 3.784 & -0.37 & 8.8 & 0.2 & 20050827 & $2017 \mathrm{~m} 15 \mathrm{~b}$ & 026 & 12.689 & 0.852 & 6092 & +4.4 & -0.5 & +0.2 & 45 & \\
\hline HD 190613 & 3.764 & 0.19 & -15.8 & 0.4 & 20050827 & $2017 \mathrm{~m} 15 \mathrm{~b}$ & 043 & -10.239 & 0.771 & 5800 & +4.3 & -0.3 & +0.2 & 55 & \\
\hline HD 192428 & 3.772 & -0.53 & 15.8 & 0.2 & 20050827 & $2017 \mathrm{~m} 15 \mathrm{~b}$ & 059 & 22.577 & 1.481 & 5794 & +3.7 & -0.9 & +0.3 & 51 & $\mathrm{cc}$ \\
\hline HD 194601 & 3.728 & -0.21 & -8.6 & 0.2 & 20050827 & $2017 \mathrm{~m} 15 \mathrm{~b}$ & 108 & -8.925 & 0.952 & 5334 & +3.5 & -0.5 & +0.1 & 71 & \\
\hline HD 194581 & 3.708 & -0.37 & -59.0 & 0.1 & 20050827 & $2017 \mathrm{~m} 15 \mathrm{~b}$ & 121 & -57.864 & 0.592 & 5315 & +3.7 & -0.5 & +0.2 & 77 & \\
\hline HD 207467 & 3.733 & -0.04 & -16.2 & 0.4 & 20050827 & $2017 \mathrm{~m} 15 \mathrm{~b}$ & 135 & -466.692 & 25.600 & 0 & +0.0 & +0.0 & +0.0 & 00 & $\mathrm{n}$ \\
\hline HD 192772 & 3.802 & -0.34 & 12.7 & 0.3 & 20050827 & $2017 \mathrm{~m} 15 \mathrm{~b}$ & 148 & 23.171 & 1.067 & 5913 & +3.8 & -0.9 & +0.4 & 55 & \\
\hline HD 192628 & 3.763 & -0.30 & -53.7 & 0.2 & 20050827 & $2017 \mathrm{~m} 15$ & 007 & 51.996 & 0.741 & 5931 & +4.2 & -0.5 & +0.2 & 90 & \\
\hline HD 192266 & 3.784 & -0.37 & 8.8 & 0.2 & 20050827 & $2017 \mathrm{~m} 15$ & 026 & 8.453 & 0.570 & 6196 & +4.3 & -0.5 & +0.3 & 109 & \\
\hline HD 190613 & 3.764 & 0.19 & -15.8 & 0.4 & 20050827 & $2017 \mathrm{~m} 15$ & 043 & -16.719 & 0.775 & 5878 & +4.3 & -0.3 & +0.2 & 117 & \\
\hline HD 192428 & 3.772 & -0.53 & 15.8 & 0.2 & 20050827 & $2017 \mathrm{~m} 15$ & 059 & 16.577 & 1.363 & 5906 & +4.0 & -0.9 & +0.3 & 92 & \\
\hline HD 192117 & 3.731 & -0.07 & 30.9 & 0.3 & 20050827 & $2017 \mathrm{~m} 15$ & 075 & 755.172 & 9.722 & 0 & +0.0 & +0.0 & +0.0 & 0 & $\mathrm{n}$ \\
\hline HD 194601 & 3.728 & -0.21 & -8.6 & 0.2 & 20050827 & $2017 \mathrm{~m} 15$ & 108 & -8.509 & 0.692 & 5229 & +3.3 & -0.7 & +0.2 & 120 & \\
\hline HD 194581 & 3.708 & -0.37 & -59.0 & 0.1 & 20050827 & $2017 \mathrm{~m} 15$ & 121 & -58.443 & 0.595 & 5464 & +3.8 & -0.3 & +0.1 & 148 & \\
\hline HD 207467 & 3.733 & -0.04 & -16.2 & 0.4 & 20050827 & $2017 \mathrm{~m} 15$ & 135 & -45.205 & 4.302 & 0 & +0.0 & +0.0 & +0.0 & 0 & $\mathrm{n}$ \\
\hline HD 192772 & 3.802 & -0.34 & 12.7 & 0.3 & 20050827 & $2017 \mathrm{~m} 15$ & 148 & 13.494 & 0.655 & 6114 & +4.0 & -0.7 & +0.3 & 132 & \\
\hline
\end{tabular}


Table 14

(Continued)

\begin{tabular}{|c|c|c|c|c|c|c|c|c|c|c|c|c|c|c|c|}
\hline \multirow[t]{2}{*}{ Name } & \multicolumn{4}{|c|}{ GCS } & \multicolumn{11}{|c|}{ RAVE values } \\
\hline & $\log \left(T_{\text {eff }}\right)$ & {$[\mathrm{Fe} / \mathrm{H}]$} & HRV & eHRV & Obsdate & FieldName & FibNum & HRV & eHRV & $T_{\text {eff }}$ & $\log g$ & Met & $\alpha$ & $\mathrm{S} 2 \mathrm{~N}$ & SpectraFLAG \\
\hline HD 216531 & 3.764 & -0.26 & -2.8 & 0.2 & 20050827 & $2255 \mathrm{~m} 44 \mathrm{~b}$ & 002 & 12.698 & 2.510 & 6880 & +4.8 & +0.2 & +0.0 & 18 & $\mathrm{cc}$ \\
\hline HD 215468 & 3.832 & -0.31 & -16.5 & 1.0 & 20050827 & $2255 \mathrm{~m} 44 \mathrm{~b}$ & 016 & -13.316 & 1.895 & 6801 & +4.0 & -0.5 & +0.2 & 35 & \\
\hline HD 215877 & 3.753 & -0.20 & 37.8 & 0.3 & 20050827 & $2255 \mathrm{~m} 44 \mathrm{~b}$ & 028 & 37.065 & 0.991 & 5843 & +4.4 & -0.4 & +0.2 & 48 & \\
\hline HD 216568 & 3.799 & -0.47 & 27.0 & 0.3 & 20050827 & $2255 \mathrm{~m} 44 \mathrm{~b}$ & 075 & 20.612 & 1.460 & 6171 & +3.8 & -0.6 & +0.0 & 48 & $\mathrm{cc}$ \\
\hline HD 217025 & 3.714 & 0.01 & -7.2 & 0.3 & 20050827 & $2255 \mathrm{~m} 44 \mathrm{~b}$ & 082 & -14.490 & 1.079 & 5428 & +4.7 & -0.1 & +0.0 & 54 & \\
\hline HD 217844 & 3.792 & -0.11 & 44.0 & 0.3 & 20050827 & $2255 \mathrm{~m} 44 \mathrm{~b}$ & 139 & 50.159 & 0.695 & 6104 & +3.8 & -0.4 & +0.2 & 69 & \\
\hline HD 216531 & 3.764 & -0.26 & -2.8 & 0.2 & 20050827 & $2255 \mathrm{~m} 44$ & 002 & -2.133 & 0.955 & 5988 & +4.6 & -0.6 & +0.4 & 57 & \\
\hline HD 215468 & 3.832 & -0.31 & -16.5 & 1.0 & 20050827 & $2255 \mathrm{~m} 44$ & 016 & -14.253 & 0.795 & 6821 & +4.0 & -0.4 & +0.2 & 141 & \\
\hline HD 215877 & 3.753 & -0.20 & 37.8 & 0.3 & 20050827 & $2255 \mathrm{~m} 44$ & 028 & 38.380 & 1.060 & 5922 & +4.4 & -0.3 & +0.2 & 114 & \\
\hline HD 216568 & 3.799 & -0.47 & 27.0 & 0.3 & 20050827 & $2255 \mathrm{~m} 44$ & 075 & 28.317 & 1.005 & 6105 & +3.9 & -0.9 & +0.4 & 127 & \\
\hline HD 217025 & 3.714 & 0.01 & -7.2 & 0.3 & 20050827 & $2255 \mathrm{~m} 44$ & 082 & -7.593 & 1.083 & 5447 & +4.7 & -0.0 & +0.0 & 92 & \\
\hline HD 217844 & 3.792 & -0.11 & 44.0 & 0.3 & 20050827 & $2255 \mathrm{~m} 44$ & 139 & 45.229 & 0.485 & 5950 & +3.7 & -0.5 & +0.3 & 129 & \\
\hline HD 223121 & 3.697 & 0.10 & -17.6 & 0.3 & 20050827 & $2348 \mathrm{~m} 33 \mathrm{~b}$ & 072 & -13.219 & 1.617 & 4808 & +3.6 & -0.2 & +0.0 & 23 & \\
\hline HD 223723 & 3.763 & -0.19 & 4.3 & 0.2 & 20050827 & $2348 \mathrm{~m} 33 \mathrm{~b}$ & 080 & 7.586 & 1.360 & 5940 & +4.5 & -0.1 & +0.2 & 46 & \\
\hline HD 223691 & 3.733 & -0.17 & 1.6 & 0.2 & 20050827 & $2348 \mathrm{~m} 33 \mathrm{~b}$ & 113 & 0.946 & 0.951 & 5558 & +4.1 & -0.4 & +0.2 & 76 & \\
\hline HD 223641 & 3.728 & -0.26 & 14.3 & 0.2 & 20050827 & $2348 \mathrm{~m} 33 \mathrm{~b}$ & 147 & 16.481 & 1.132 & 5440 & +3.6 & -0.7 & +0.3 & 63 & \\
\hline HD 223121 & 3.697 & 0.10 & -17.6 & 0.3 & 20050827 & $2348 \mathrm{~m} 33$ & 072 & -16.241 & 1.198 & 5036 & +4.4 & -0.1 & +0.1 & 62 & $\mathrm{cc}$ \\
\hline HD 223723 & 3.763 & -0.19 & 4.3 & 0.2 & 20050827 & $2348 \mathrm{~m} 33$ & 080 & 4.062 & 1.316 & 6432 & +4.7 & +0.1 & +0.0 & 102 & \\
\hline HD 223691 & 3.733 & -0.17 & 1.6 & 0.2 & 20050827 & $2348 \mathrm{~m} 33$ & 113 & 1.667 & 0.531 & 5468 & +3.8 & -0.4 & +0.2 & 130 & \\
\hline HD 223641 & 3.728 & -0.26 & 14.3 & 0.2 & 20050827 & $2348 \mathrm{~m} 33$ & 147 & 14.033 & 0.801 & 5394 & +3.7 & -0.7 & +0.3 & 101 & \\
\hline HD 4989 & 3.704 & -0.24 & 1.6 & 0.3 & 20050828 & $0103 \mathrm{~m} 42 \mathrm{~b}$ & 027 & 6.112 & 1.275 & 4919 & +4.4 & -0.7 & +0.2 & 46 & \\
\hline HD 5510 & 3.791 & 0.09 & -4.2 & 0.3 & 20050828 & $0103 m 42 b$ & 048 & 0.977 & 1.239 & 6092 & +4.2 & -0.3 & +0.2 & 62 & \\
\hline HD 7052 & 3.717 & -0.08 & 10.1 & 0.3 & 20050828 & $0103 \mathrm{~m} 42 \mathrm{~b}$ & 089 & 8.378 & 1.568 & 5395 & +4.7 & +0.0 & +0.0 & 28 & $\mathrm{t}$ \\
\hline HD 6444 & 3.837 & -0.09 & -7.2 & 0.7 & 20050828 & $0103 \mathrm{~m} 42 \mathrm{~b}$ & 121 & -10.387 & 1.126 & 6497 & +3.9 & -0.4 & +0.2 & 74 & \\
\hline HD 6768 & 3.818 & -0.12 & -4.5 & 2.0 & 20050828 & $0103 \mathrm{~m} 42 \mathrm{~b}$ & 142 & 1.099 & 1.330 & 6252 & +3.6 & -0.5 & +0.4 & 56 & $\mathrm{t}$ \\
\hline HD 4989 & 3.704 & -0.24 & 1.6 & 0.3 & 20050828 & $0103 \mathrm{~m} 42$ & 027 & 3.216 & 1.126 & 5320 & +4.7 & -0.3 & +0.0 & 103 & \\
\hline HD 5510 & 3.791 & 0.09 & -4.2 & 0.3 & 20050828 & $0103 \mathrm{~m} 42$ & 048 & -3.262 & 0.998 & 5831 & +3.8 & -0.5 & +0.3 & 123 & \\
\hline HD 7052 & 3.717 & -0.08 & 10.1 & 0.3 & 20050828 & $0103 \mathrm{~m} 42$ & 089 & 10.239 & 0.923 & 5448 & +4.8 & -0.1 & +0.0 & 70 & \\
\hline HD 6444 & 3.837 & -0.09 & -7.2 & 0.7 & 20050828 & $0103 \mathrm{~m} 42$ & 121 & -8.370 & 0.966 & 6586 & +4.0 & -0.4 & +0.2 & 150 & \\
\hline HD 6768 & 3.818 & -0.12 & -4.5 & 2.0 & 20050828 & $0103 \mathrm{~m} 42$ & 142 & -2.720 & 0.581 & 6163 & +3.4 & -0.6 & +0.4 & 110 & \\
\hline HD 10166 & 3.717 & -0.40 & -1.8 & 0.3 & 20050828 & $0145 \mathrm{~m} 25 \mathrm{~b}$ & 012 & -4.625 & 2.978 & 5341 & +4.4 & +0.1 & +0.1 & 16 & $\mathrm{cc}$ \\
\hline HD 10037 & 3.774 & -0.50 & -20.5 & 0.3 & 20050828 & $0145 \mathrm{~m} 25 \mathrm{~b}$ & 028 & -22.128 & 1.262 & 5729 & +3.7 & -0.8 & +0.3 & 56 & \\
\hline HD 9769 & 3.786 & -0.33 & 31.5 & 0.4 & 20050828 & $0145 \mathrm{~m} 25 \mathrm{~b}$ & 054 & 29.559 & 0.955 & 5958 & +3.9 & -0.6 & +0.3 & 52 & \\
\hline HD 11523 & 3.760 & -0.23 & 20.6 & 0.2 & 20050828 & $0145 \mathrm{~m} 25 \mathrm{~b}$ & 138 & 18.261 & 1.035 & 5834 & +4.4 & -0.5 & +0.3 & 58 & \\
\hline HD 11020 & 3.721 & -0.06 & 22.2 & 0.3 & 20050828 & $0145 \mathrm{~m} 25 \mathrm{~b}$ & 144 & 20.625 & 1.125 & 5701 & +4.6 & -0.2 & +0.1 & 45 & \\
\hline HD 10166 & 3.717 & -0.40 & -1.8 & 0.3 & 20050828 & $0145 \mathrm{~m} 25$ & 012 & -1.670 & 0.892 & 5236 & +4.4 & -0.4 & +0.0 & 55 & \\
\hline HD 10037 & 3.774 & -0.50 & -20.5 & 0.3 & 20050828 & $0145 \mathrm{~m} 25$ & 028 & -22.784 & 0.633 & 5995 & +3.9 & -0.7 & +0.3 & 140 & \\
\hline HD 9769 & 3.786 & -0.33 & 31.5 & 0.4 & 20050828 & $0145 \mathrm{~m} 25$ & 054 & 30.835 & 0.748 & 6162 & +4.1 & -0.5 & +0.2 & 121 & \\
\hline HD 11523 & 3.760 & -0.23 & 20.6 & 0.2 & 20050828 & $0145 \mathrm{~m} 25$ & 138 & 19.082 & 0.838 & 5848 & +4.4 & -0.5 & +0.3 & 118 & \\
\hline HD 11020 & 3.721 & -0.06 & 22.2 & 0.3 & 20050828 & $0145 \mathrm{~m} 25$ & 144 & 22.150 & 0.965 & 5574 & +4.7 & -0.3 & +0.1 & 108 & \\
\hline HD 14680 & 3.699 & -0.03 & 51.5 & 0.2 & 20050828 & $0230 \mathrm{~m} 30$ & 026 & 50.609 & 1.377 & 5159 & +4.8 & -0.2 & +0.0 & 68 & \\
\hline HD 14868 & 3.760 & -0.17 & 28.8 & 0.3 & 20050828 & $0230 \mathrm{~m} 30$ & 058 & 32.101 & 0.653 & 5885 & +4.4 & -0.4 & +0.3 & 115 & \\
\hline HD 15337 & 3.707 & 0.14 & -4.6 & 0.3 & 20050828 & $0230 \mathrm{~m} 30$ & 075 & -1.753 & 1.200 & 5223 & +4.5 & -0.2 & +0.2 & 112 & \\
\hline HD 16297 & 3.726 & -0.03 & -1.7 & 0.2 & 20050828 & $0230 \mathrm{~m} 30$ & 090 & -0.629 & 1.428 & 5765 & +4.8 & -0.0 & +0.0 & 79 & \\
\hline HD 16784 & 3.772 & -0.54 & 40.3 & 0.3 & 20050828 & $0230 \mathrm{~m} 30$ & 114 & 40.749 & 1.089 & 6280 & +4.7 & -0.6 & +0.3 & 47 & \\
\hline HD 169812 & 3.771 & -0.15 & -59.5 & 0.2 & 20050828 & $1830 \mathrm{~m} 40 \mathrm{~b}$ & 013 & -59.071 & 2.344 & 6091 & +3.9 & -0.2 & +0.0 & 19 & $\mathrm{cc}$ \\
\hline HD 169499 & 3.708 & -0.47 & -15.0 & 0.2 & 20050828 & $1830 \mathrm{~m} 40 \mathrm{~b}$ & 016 & -12.065 & 2.377 & 5591 & +4.1 & -0.2 & +0.0 & 33 & \\
\hline HD 170865 & 3.755 & -0.14 & 52.2 & 0.2 & 20050828 & $1830 \mathrm{~m} 40 \mathrm{~b}$ & 079 & 55.214 & 1.208 & 6112 & +4.7 & -0.4 & +0.3 & 68 & \\
\hline HD 170869 & 3.757 & -0.30 & -63.8 & 0.3 & 20050828 & $1830 \mathrm{~m} 40 \mathrm{~b}$ & 098 & -63.249 & 0.892 & 6103 & +4.1 & -0.2 & +0.1 & 49 & \\
\hline HD 172283 & 3.795 & -0.37 & -22.5 & 0.3 & 20050828 & $1830 \mathrm{~m} 40 \mathrm{~b}$ & 130 & -20.023 & 2.155 & 6190 & +3.8 & -0.6 & +0.2 & 39 & $\mathrm{cc}$ \\
\hline HD 169812 & 3.771 & -0.15 & -59.5 & 0.2 & 20050828 & 1830m40 & 013 & -58.058 & 0.894 & 5847 & +4.0 & -0.5 & +0.3 & 71 & \\
\hline HD 169499 & 3.708 & -0.47 & -15.0 & 0.2 & 20050828 & $1830 \mathrm{~m} 40$ & 016 & -12.742 & 1.772 & 5420 & +3.5 & -0.6 & +0.1 & 99 & \\
\hline HD 170865 & 3.755 & -0.14 & 52.2 & 0.2 & 20050828 & $1830 \mathrm{~m} 40$ & 07 & 53.598 & 0.636 & 5821 & +4.2 & -0.6 & +0.3 & 150 & \\
\hline HD 170869 & 3.757 & -0.30 & -63.8 & 0.3 & 20050828 & $1830 \mathrm{~m} 40$ & 098 & -63.634 & 0.523 & 5876 & +3.9 & -0.5 & +0.2 & 115 & \\
\hline HD 172283 & 3.795 & -0.37 & -22.5 & 0.3 & 20050828 & $1830 \mathrm{~m} 40$ & 130 & -21.514 & 1.899 & 6390 & +3.9 & -0.7 & +0.4 & 103 & \\
\hline HD 175114 & 3.801 & -0.47 & 30.9 & 0.4 & 20050828 & $1900 \mathrm{~m} 30 \mathrm{~b}$ & 007 & 29.620 & 1.040 & 5844 & +3.3 & -1.1 & +0.3 & 43 & \\
\hline HD 173858 & 3.762 & -0.58 & 66.0 & 0.2 & 20050828 & $1900 \mathrm{~m} 30 \mathrm{~b}$ & 044 & 67.152 & 1.229 & 6169 & +4.5 & -0.4 & +0.2 & 50 & \\
\hline HD 175568 & 3.776 & -0.32 & -20.3 & 0.3 & 20050828 & $1900 \mathrm{~m} 30 \mathrm{~b}$ & 072 & -22.102 & 0.985 & 6187 & +4.3 & -0.3 & +0.1 & 49 & \\
\hline HD 175979 & 3.774 & -0.40 & -1.3 & 0.3 & 20050828 & $1900 \mathrm{~m} 30 \mathrm{~b}$ & 075 & -3.173 & 0.830 & 6054 & +4.0 & -0.4 & +0.1 & 68 & \\
\hline HD 176367 & 3.778 & 0.11 & -5.8 & 0.5 & 20050828 & $1900 \mathrm{~m} 30 \mathrm{~b}$ & 078 & -7.187 & 2.102 & 6252 & +4.7 & -0.2 & +0.1 & 70 & $\mathrm{cc}$ \\
\hline HD 176612 & 3.774 & -0.29 & -16.7 & 0.3 & 20050828 & $1900 \mathrm{~m} 30 \mathrm{~b}$ & 082 & -18.859 & 0.870 & 6231 & +4.3 & -0.4 & +0.2 & 58 & \\
\hline HD 177033 & 3.679 & -0.23 & -46.9 & 0.3 & 20050828 & $1900 \mathrm{~m} 30 \mathrm{~b}$ & 085 & -48.394 & 0.990 & 4861 & +4.6 & -0.1 & +0.0 & 43 & \\
\hline HD 178673 & 3.772 & -0.27 & 27.6 & 0.3 & 20050828 & $1900 \mathrm{~m} 30 \mathrm{~b}$ & 112 & 26.270 & 1.073 & 6691 & +4.3 & -0.0 & +0.2 & 66 & \\
\hline HD 177122 & 3.768 & -0.31 & -31.6 & 0.2 & 20050828 & $1900 \mathrm{~m} 30 \mathrm{~b}$ & 139 & -33.593 & 0.528 & 5953 & +4.4 & -0.5 & +0.3 & 86 & \\
\hline
\end{tabular}


Table 14

(Continued)

\begin{tabular}{|c|c|c|c|c|c|c|c|c|c|c|c|c|c|c|c|}
\hline \multirow[t]{2}{*}{ Name } & \multicolumn{4}{|c|}{ GCS } & \multicolumn{11}{|c|}{ RAVE values } \\
\hline & $\log \left(T_{\text {eff }}\right)$ & {$[\mathrm{Fe} / \mathrm{H}]$} & HRV & eHRV & Obsdate & FieldName & FibNum & HRV & eHRV & $T_{\text {eff }}$ & $\log g$ & Met & $\alpha$ & $\mathrm{S} 2 \mathrm{~N}$ & SpectraFLAG \\
\hline HD 175114 & 3.801 & -0.47 & 30.9 & 0.4 & 20050828 & $1900 \mathrm{~m} 30$ & 007 & 29.692 & 0.767 & 6091 & +3.8 & -0.9 & +0.4 & 101 & \\
\hline HD 173858 & 3.762 & -0.58 & 66.0 & 0.2 & 20050828 & $1900 \mathrm{~m} 30$ & 044 & 65.839 & 0.831 & 6125 & +4.4 & -0.5 & +0.3 & 108 & \\
\hline HD 175568 & 3.776 & -0.32 & -20.3 & 0.3 & 20050828 & $1900 \mathrm{~m} 30$ & 072 & -21.259 & 0.658 & 5760 & +4.1 & -0.6 & +0.2 & 108 & \\
\hline HD 175979 & 3.774 & -0.40 & -1.3 & 0.3 & 20050828 & $1900 \mathrm{~m} 30$ & 075 & -2.194 & 0.495 & 6016 & +3.7 & -0.5 & +0.1 & 137 & \\
\hline HD 176367 & 3.778 & 0.11 & -5.8 & 0.5 & 20050828 & $1900 \mathrm{~m} 30$ & 078 & -6.836 & 2.214 & 6439 & +4.8 & -0.1 & +0.1 & 119 & $\mathrm{cc}$ \\
\hline HD 176612 & 3.774 & -0.29 & -16.7 & 0.3 & 20050828 & $1900 \mathrm{~m} 30$ & 082 & -17.171 & 0.592 & 6224 & +4.5 & -0.3 & +0.1 & 121 & \\
\hline HD 177033 & 3.679 & -0.23 & -46.9 & 0.3 & 20050828 & $1900 \mathrm{~m} 30$ & 085 & -48.422 & 0.562 & 4889 & +4.6 & -0.3 & +0.0 & 112 & \\
\hline HD 178673 & 3.772 & -0.27 & 27.6 & 0.3 & 20050828 & $1900 \mathrm{~m} 30$ & 112 & 26.467 & 0.928 & 6416 & +4.2 & -0.2 & +0.3 & 120 & \\
\hline HD 177122 & 3.768 & -0.31 & -31.6 & 0.2 & 20050828 & $1900 \mathrm{~m} 30$ & 139 & -33.000 & 0.503 & 6048 & +4.6 & -0.5 & +0.3 & 165 & \\
\hline HD 189389 & 3.761 & 0.03 & 14.3 & 0.3 & 20050828 & $2005 \mathrm{~m} 43$ & 044 & 13.769 & 1.223 & 5837 & +4.1 & -0.2 & +0.3 & 74 & \\
\hline HD 188903 & 3.782 & 0.01 & -3.3 & 0.3 & 20050828 & $2005 \mathrm{~m} 43$ & 058 & -2.607 & 0.737 & 6061 & +4.0 & -0.3 & +0.2 & 109 & \\
\hline HD 190649 & 3.745 & -0.47 & -55.7 & 0.2 & 20050828 & $2005 \mathrm{~m} 43$ & 079 & -55.229 & 1.591 & 6194 & +4.5 & -0.3 & +0.3 & 102 & \\
\hline HD 192071 & 3.752 & -0.45 & -16.4 & 0.3 & 20050828 & $2005 \mathrm{~m} 43$ & 129 & -16.474 & 0.663 & 5730 & +3.9 & -0.7 & +0.3 & 138 & \\
\hline HD 190269 & 3.815 & -0.06 & 1.9 & 0.2 & 20050828 & $2005 \mathrm{~m} 43$ & 149 & -0.640 & 0.647 & 6322 & +4.0 & -0.3 & +0.2 & 111 & \\
\hline HD 205187 & 3.769 & -0.18 & 24.2 & 0.2 & 20050828 & $2142 \mathrm{~m} 41 \mathrm{~b}$ & 062 & 22.655 & 0.924 & 5922 & +4.3 & -0.4 & +0.3 & 63 & \\
\hline HD 207790 & 3.778 & 0.14 & 28.3 & 1.0 & 20050828 & $2142 \mathrm{~m} 41 \mathrm{~b}$ & 104 & 24.974 & 1.908 & 6257 & +4.4 & -0.2 & +0.3 & 64 & \\
\hline HD 206303 & 3.790 & -0.19 & -14.3 & 0.6 & 20050828 & $2142 \mathrm{~m} 41 \mathrm{~b}$ & 121 & -14.624 & 0.987 & 6412 & +4.0 & -0.1 & +0.2 & 65 & \\
\hline HD 206667 & 3.767 & -0.26 & 19.3 & 0.2 & 20050828 & $2142 \mathrm{~m} 41 \mathrm{~b}$ & 135 & 19.705 & 0.650 & 6162 & +5.0 & -0.2 & +0.2 & 81 & \\
\hline HD 206682 & 3.792 & -0.15 & 9.2 & 0.3 & 20050828 & $2142 \mathrm{~m} 41 \mathrm{~b}$ & 148 & 13.098 & 1.327 & 6403 & +4.8 & -0.3 & +0.3 & 57 & \\
\hline HD 205187 & 3.769 & -0.18 & 24.2 & 0.2 & 20050828 & $2142 \mathrm{~m} 41$ & 062 & 23.812 & 0.578 & 5971 & +4.3 & -0.3 & +0.3 & 146 & \\
\hline HD 207790 & 3.778 & 0.14 & 28.3 & 1.0 & 20050828 & $2142 \mathrm{~m} 41$ & 104 & 27.269 & 1.118 & 6102 & +4.0 & -0.2 & +0.2 & 137 & \\
\hline HD 206303 & 3.790 & -0.19 & -14.3 & 0.6 & 20050828 & $2142 \mathrm{~m} 41$ & 121 & -14.083 & 0.786 & 6401 & +4.0 & -0.1 & +0.2 & 129 & \\
\hline HD 206667 & 3.767 & -0.26 & 19.3 & 0.2 & 20050828 & $2142 \mathrm{~m} 41$ & 135 & 18.444 & 0.709 & 6165 & +4.9 & -0.3 & +0.2 & 155 & \\
\hline HD 206682 & 3.792 & -0.15 & 9.2 & 0.3 & 20050828 & $2142 \mathrm{~m} 41$ & 148 & 9.145 & 0.950 & 6202 & +4.3 & -0.3 & +0.3 & 118 & \\
\hline HD 2404 & 3.724 & -0.44 & -43.1 & 0.3 & 20050829 & $0030 \mathrm{~m} 31$ & 005 & -49.767 & 1.178 & 5416 & +4.1 & -0.7 & +0.3 & 49 & \\
\hline HD 2348 & 3.787 & -0.40 & 32.5 & 0.3 & 20050829 & $0030 \mathrm{~m} 31$ & 021 & 31.169 & 1.363 & 6186 & +4.3 & -0.4 & +0.2 & 47 & \\
\hline HD 1557 & 3.798 & -0.45 & 25.1 & 0.3 & 20050829 & $0030 \mathrm{~m} 31$ & 043 & 23.863 & 1.498 & 6289 & +3.9 & -0.6 & +0.3 & 37 & \\
\hline HD 1674 & 3.784 & -0.17 & -5.7 & 0.7 & 20050829 & $0030 \mathrm{~m} 31$ & 057 & -4.178 & 0.814 & 5850 & +4.0 & -0.6 & +0.3 & 62 & \\
\hline HD 3810 & 3.750 & -0.31 & 37.4 & 0.2 & 20050829 & $0030 \mathrm{~m} 31$ & 101 & 36.477 & 1.159 & 5963 & +4.6 & -0.4 & +0.2 & 49 & \\
\hline HD 3560 & 3.788 & -0.31 & -10.6 & 0.4 & 20050829 & $0030 \mathrm{~m} 31$ & 127 & -12.069 & 1.317 & 6167 & +3.9 & -0.3 & +0.1 & 30 & \\
\hline HD 157884 & 3.804 & -0.38 & 9.1 & 0.5 & 20050829 & $1730 \mathrm{~m} 30$ & 003 & 12.029 & 0.593 & 6651 & +5.0 & -0.3 & +0.2 & 144 & \\
\hline HD 156423 & 3.719 & -0.34 & -30.2 & 0.3 & 20050829 & $1730 \mathrm{~m} 30$ & 043 & -31.890 & 0.843 & 5199 & +4.1 & -0.6 & +0.2 & 106 & \\
\hline HD 160573 & 3.799 & -0.10 & 3.7 & 1.4 & 20050829 & $1730 \mathrm{~m} 30$ & 118 & 8.937 & 1.111 & 6287 & +3.6 & -0.5 & +0.2 & 132 & \\
\hline HD 159784 & 3.781 & 0.32 & -8.8 & 0.3 & 20050829 & $1730 \mathrm{~m} 30$ & 127 & -8.392 & 1.008 & 6226 & +4.2 & +0.0 & +0.2 & 114 & \\
\hline HD 159882 & 3.729 & -0.15 & 12.3 & 0.3 & 20050829 & $1730 \mathrm{~m} 30$ & 136 & 12.660 & 0.897 & 5597 & +4.8 & -0.2 & +0.0 & 111 & \\
\hline HD 158884 & 3.789 & -0.48 & 53.7 & 0.5 & 20050829 & $1730 \mathrm{~m} 30$ & 144 & 54.156 & 0.509 & 5979 & +3.6 & -0.9 & +0.4 & 118 & \\
\hline HD 157884 & 3.804 & -0.38 & 9.1 & 0.5 & 20050829 & $1930 \mathrm{~m} 35 \mathrm{~b}$ & 002 & 18.933 & 3.780 & 6090 & +4.5 & +0.1 & +0.1 & 12 & $\mathrm{cc}$ \\
\hline HD 156423 & 3.719 & -0.34 & -30.2 & 0.3 & 20050829 & $1930 \mathrm{~m} 35 \mathrm{~b}$ & 012 & -32.357 & 1.672 & 6278 & +3.7 & -0.6 & +0.3 & 54 & \\
\hline HD 160573 & 3.799 & -0.10 & 3.7 & 1.4 & 20050829 & $1930 \mathrm{~m} 35 \mathrm{~b}$ & 026 & 23.692 & 1.639 & 5874 & +4.4 & -0.4 & +0.3 & 52 & \\
\hline HD 159784 & 3.781 & 0.32 & -8.8 & 0.3 & 20050829 & $1930 \mathrm{~m} 35 \mathrm{~b}$ & 106 & 17.940 & 1.340 & 6050 & +3.9 & -0.1 & +0.2 & 45 & \\
\hline HD 159882 & 3.729 & -0.15 & 12.3 & 0.3 & 20050829 & $1930 \mathrm{~m} 35 \mathrm{~b}$ & 121 & -6.329 & 0.824 & 5964 & +4.6 & -0.0 & +0.0 & 38 & \\
\hline HD 158884 & 3.789 & -0.48 & 53.7 & 0.5 & 20050829 & $1930 \mathrm{~m} 35 \mathrm{~b}$ & 140 & 5.617 & 1.068 & 5635 & +4.6 & -0.0 & +0.0 & 37 & \\
\hline HD 183198 & 3.725 & -0.09 & -7.6 & 0.3 & 20050829 & 1930m35 & 002 & -9.661 & 2.264 & 5459 & +4.8 & -0.1 & +0.1 & 37 & $\mathrm{t}$ \\
\hline HD 181893 & 3.810 & -0.21 & -42.1 & 0.6 & 20050829 & $1930 \mathrm{~m} 35$ & 012 & -42.955 & 0.908 & 6270 & +3.9 & -0.6 & +0.4 & 133 & \\
\hline HD 181452 & 3.749 & -0.27 & 25.8 & 0.2 & 20050829 & $1930 \mathrm{~m} 35$ & 026 & 23.519 & 1.576 & 6267 & +4.6 & +0.0 & +0.1 & 110 & $\mathrm{cc}$ \\
\hline HD 184514 & 3.769 & 0.00 & 10.4 & 0.3 & 20050829 & $1930 \mathrm{~m} 35$ & 106 & 10.729 & 1.162 & 5866 & +3.7 & -0.2 & +0.2 & 100 & \\
\hline HD 185679 & 3.745 & 0.06 & -10.8 & 0.2 & 20050829 & $1930 \mathrm{~m} 35$ & 121 & -10.358 & 0.967 & 5949 & +4.6 & -0.1 & +0.1 & 88 & \\
\hline HD 184374 & 3.737 & 0.11 & 15.8 & 0.3 & 20050829 & 1930m35 & 140 & 16.675 & 1.049 & 5790 & +4.9 & -0.0 & +0.0 & 91 & \\
\hline HD 199903 & 3.760 & -0.07 & 42.0 & 0.2 & 20050829 & $2100 \mathrm{~m} 35 \mathrm{~b}$ & 034 & 56.086 & 1.128 & 5924 & +4.3 & -0.3 & +0.2 & 80 & \\
\hline HD 198697 & 3.744 & -0.05 & 3.0 & 0.3 & 20050829 & $2100 \mathrm{~m} 35 \mathrm{~b}$ & 044 & 19.458 & 0.842 & 6085 & +4.6 & -0.1 & +0.1 & 60 & \\
\hline HD 199672 & 3.787 & 0.00 & -15.1 & 0.3 & 20050829 & $2100 \mathrm{~m} 35 \mathrm{~b}$ & 075 & -4.957 & 0.735 & 5806 & +3.3 & -0.4 & +0.2 & 71 & \\
\hline HD 200382 & 3.703 & 0.16 & 16.0 & 0.4 & 20050829 & $2100 \mathrm{~m} 35 \mathrm{~b}$ & 108 & 11.064 & 0.928 & 5062 & +4.4 & +0.1 & +0.0 & 37 & \\
\hline HD 201513 & 3.735 & -0.17 & 20.0 & 0.3 & 20050829 & $2100 \mathrm{~m} 35 \mathrm{~b}$ & 113 & 16.486 & 1.698 & 6277 & +4.8 & +0.1 & +0.1 & 26 & \\
\hline HD 200608 & 3.760 & 0.10 & 13.1 & 0.4 & 20050829 & $2100 \mathrm{~m} 35 \mathrm{~b}$ & 139 & 18.234 & 0.901 & 5762 & +4.1 & -0.4 & +0.3 & 70 & \\
\hline HD 200344 & 3.775 & -0.08 & 49.1 & 0.3 & 20050829 & $2100 \mathrm{~m} 35 \mathrm{~b}$ & 144 & 62.212 & 0.981 & 5833 & +3.6 & -0.5 & +0.3 & 61 & \\
\hline HD 199903 & 3.760 & -0.07 & 42.0 & 0.2 & 20050829 & $2100 \mathrm{~m} 35$ & 034 & 42.375 & 0.578 & 6047 & +4.6 & -0.2 & +0.2 & 147 & \\
\hline HD 198697 & 3.744 & -0.05 & 3.0 & 0.3 & 20050829 & $2100 \mathrm{~m} 35$ & 044 & 2.443 & 0.676 & 5934 & +4.7 & -0.3 & +0.2 & 126 & \\
\hline HD 199672 & 3.787 & 0.00 & -15.1 & 0.3 & 20050829 & $2100 \mathrm{~m} 35$ & 075 & -14.549 & 0.690 & 5926 & +3.5 & -0.3 & +0.2 & 136 & \\
\hline HD 200382 & 3.703 & 0.16 & 16.0 & 0.4 & 20050829 & $2100 \mathrm{~m} 35$ & 108 & 16.506 & 0.838 & 5099 & +4.4 & -0.0 & +0.0 & 91 & \\
\hline HD 201513 & 3.735 & -0.17 & 20.0 & 0.3 & 20050829 & $2100 \mathrm{~m} 35$ & 113 & 19.251 & 0.946 & 5976 & +4.7 & -0.2 & +0.1 & 79 & \\
\hline HD 200608 & 3.760 & 0.10 & 13.1 & 0.4 & 20050829 & $2100 \mathrm{~m} 35$ & 139 & 10.805 & 0.663 & 5777 & +4.1 & -0.3 & +0.2 & 136 & \\
\hline HD 200344 & 3.775 & -0.08 & 49.1 & 0.3 & 20050829 & $2100 \mathrm{~m} 35$ & 144 & 48.946 & 0.680 & 5911 & +3.8 & -0.4 & +0.3 & 110 & \\
\hline HD 217221 & 3.711 & 0.16 & 26.2 & 0.1 & 20050829 & $2305 \mathrm{~m} 29 \mathrm{~b}$ & 012 & 39.502 & 1.168 & 5445 & +4.5 & -0.0 & +0.0 & 47 & \\
\hline HD 217123 & 3.789 & -0.27 & -10.7 & 0.4 & 20050829 & $2305 \mathrm{~m} 29 \mathrm{~b}$ & 049 & -20.252 & 1.313 & 6308 & +4.2 & -0.4 & +0.3 & 59 & \\
\hline
\end{tabular}


Table 14

(Continued)

\begin{tabular}{|c|c|c|c|c|c|c|c|c|c|c|c|c|c|c|c|}
\hline \multirow[t]{2}{*}{ Name } & \multicolumn{4}{|c|}{ GCS } & \multicolumn{11}{|c|}{ RAVE values } \\
\hline & $\log \left(T_{\text {eff }}\right)$ & {$[\mathrm{Fe} / \mathrm{H}]$} & HRV & eHRV & Obsdate & FieldName & FibNum & HRV & eHRV & $T_{\text {eff }}$ & $\log g$ & Met & $\alpha$ & $\mathrm{S} 2 \mathrm{~N}$ & SpectraFLAG \\
\hline HD 217500 & 3.729 & -0.12 & 0.0 & 0.3 & 20050829 & $2305 \mathrm{~m} 29 \mathrm{~b}$ & 066 & -2.920 & 1.484 & 5849 & +4.0 & -0.2 & +0.1 & 22 & $\mathrm{cc}$ \\
\hline HD 218532 & 3.765 & 0.09 & 35.4 & 0.2 & 20050829 & $2305 \mathrm{~m} 29 \mathrm{~b}$ & 085 & 38.136 & 1.140 & 6029 & +4.6 & -0.3 & +0.2 & 54 & \\
\hline HD 219057 & 3.751 & -0.22 & 0.7 & 0.2 & 20050829 & $2305 \mathrm{~m} 29 \mathrm{~b}$ & 132 & -1.502 & 1.465 & 6065 & +4.8 & -0.2 & +0.2 & 43 & \\
\hline HD 217221 & 3.711 & 0.16 & 26.2 & 0.1 & 20050829 & $2305 \mathrm{~m} 29$ & 012 & 26.936 & 0.728 & 5224 & +4.5 & -0.2 & +0.1 & 100 & \\
\hline HD 217123 & 3.789 & -0.27 & -10.7 & 0.4 & 20050829 & $2305 \mathrm{~m} 29$ & 049 & -12.458 & 0.905 & 6209 & +4.0 & -0.4 & +0.3 & 127 & \\
\hline HD 217500 & 3.729 & -0.12 & 0.0 & 0.3 & 20050829 & $2305 \mathrm{~m} 29$ & 066 & -0.547 & 0.678 & 5799 & +4.2 & -0.4 & +0.1 & 51 & \\
\hline HD 218532 & 3.765 & 0.09 & 35.4 & 0.2 & 20050829 & $2305 \mathrm{~m} 29$ & 085 & 36.420 & 0.701 & 5657 & +4.0 & -0.4 & +0.2 & 109 & \\
\hline HD 219057 & 3.751 & -0.22 & 0.7 & 0.2 & 20050829 & $2305 \mathrm{~m} 29$ & 132 & 1.416 & 0.639 & 5891 & +4.5 & -0.4 & +0.3 & 79 & \\
\hline
\end{tabular}

Note. Only normal stars, which have their SpectraFLAG column empty, were used to check the temperature values.

UCAC2 counterpart within 3 arcsec search radius was identified for $94 \%$ of the spectra; many of the remaining objects have large errors in reported proper motion. Note that UCAC2 values are systematically offset from the Starnet 2 measurements.

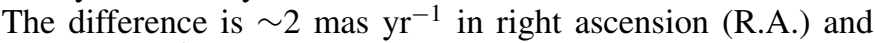

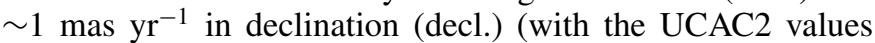
being smaller than the Starnet 2 ones). The final catalog therefore includes the UCAC2 proper motion if the Starnet 2 or Tycho-2 values are not available ( $\sim 23 \%$ of cases). The source of proper motions is flagged, so the systematic differences could be taken into account. Table 8 gives details on the use of proper motion catalogs in the present data release and their reported average and 90 percentile errors. In all cases, this data release includes proper motion from the source with the best value of reported accuracy.

\section{CONCLUSIONS}

This second data release reports radial velocities of 51,829 spectra of 49,327 different stars, randomly selected in the magnitude range of $9 \lesssim I \lesssim 12$ and located more than $25^{\circ}$ away from the Galactic plane (except for a few test observations). It covers an area of $\sim 7200 \mathrm{deg}^{2}$. These numbers approximately double the sample reported in Paper I. Moreover, this data release is the first to include values of stellar parameters as determined from stellar spectra. We report temperature, gravity, and metallicity for 21,121 normal stars, all observed after the first data release. Stars with a high rotational velocity or a peculiar type (e.g., binary stars and emission stars) will be discussed separately.

Radial velocities for stars new to this data release are more accurate than before, with typical errors between 1.3 and $1.7 \mathrm{~km}$ $\mathrm{s}^{-1}$. These values are confirmed both by repeated observations and by external datasets and have only a weak dependence on the $\mathrm{S} / \mathrm{N}$. We used five separate external datasets to check values of stellar parameters derived from the RAVE spectra. These included observations with different instruments at different resolving powers and in different wavelength regimes, as well as data from the literature. The uncertainty of stellar parameter values strongly depends on the stellar type. Despite considerable effort our calibration observations do not (yet) cover the entire parameter space. We plan to improve on this using dedicated calibration observations with at least four telescopes. For this data release we had to resort to extensive simulations which are however tuned by calibration observations. A typical RAVE star has an uncertainty of $400 \mathrm{~K}$ in temperature, 0.5 dex in gravity, and 0.2 dex in metallicity. The error depends on the $\mathrm{S} / \mathrm{N}$ and can be $\gtrsim 2$ times better/worse for stars at extremes of the noise range. Repeated observations show that these error estimates are rather conservative, possibly due to intrinsic variability of the observed stars and/or non-negligible errors of reference values from the calibration datasets.

Future data releases will follow on an approximately yearly basis. They will benefit from our considerable and ongoing effort to obtain calibration datasets using other telescopes and similar or complimentary observing techniques. Notably we expect that SkyMapper (Keller et al. 2007), an all-southern-sky survey just starting at the Siding Spring Observatory, will provide accurate photometry and temporal variability information for all RAVE stars.

RAVE is planned to observe up to a million spectra of stars away from the Galactic plane. It represents an unprecedented sample of stellar kinematics and physical properties in the range of magnitudes probing scales between the very local surveys (GCS and Famaey et al. (2005)) and more distant ones (SDSSII/SEGUE), complementing the planned AAOmega efforts closer to the Galactic plane. So it helps to complete our picture of the Milky Way, paving the way for the next-decade endeavors, like Gaia.

We are most grateful to our referee, Professor David W. Latham, for his detailed and very relevant comments which improved the quality of the presentation of the paper.

Funding for RAVE has been provided by the AngloAustralian Observatory, the Astrophysical Institute Potsdam, the Australian Research Council, the German Research foundation, the National Institute for Astrophysics at Padova, The Johns Hopkins University, the Netherlands Research School for Astronomy, the Natural Sciences and Engineering Research Council of Canada, the Slovenian Research Agency, the Swiss National Science Foundation, the National Science Foundation of the USA (AST-0508996), the Netherlands Organisation for Scientific Research, the Particle Physics and Astronomy Research Council of the UK, Opticon, Strasbourg Observatory, and the Universities of Basel, Cambridge, and Groningen. The RAVE Web site is at www.rave-survey.org.

K.C.F., Q.A.P, B.G., R.C., W.R., and E.C.W. acknowledge support from Australian Research Council grants DP0451045 and DP0772283. A.S. and E.K.G. are supported by the Swiss National Science Foundation under the grants 200020-105260 and 200020-113697. J.P.F. acknowledges support from the Keck Foundation, through a grant to JHU. R.F.G.W acknowledges seed money from the School of Arts and Sciences at JHU, plus NSF grant AST-0508996. G.M.S. was funded by a Particle Physics and Astronomy Research Council $\mathrm{PhD}$ Studentship. O.B. acknowledges financial support from the 
CNRS/INSU/PNG. P.R.F. is supported by the European Marie Curie RTN ELSA, contract MRTN-CT-2006-033481.

This research has made use of the VizieR catalog access tool, CDS, Strasbourg, France. This publication makes use of data products from the Two Micron All Sky Survey, which is a joint project of the University of Massachusetts and the IPAC/Caltech, funded by NASA and NSF. The results are based partly on observations obtained at the Asiago $1.82 \mathrm{~m}$ telescope (Italy), and at the Observatoire de Haute Provence (OHP) (France) which is operated by the French CNRS. The cross-identification of the RAVE data release with the UCAC2 catalogue was done using the electronic version of the UCAC2 kindly provided by Norbert Zacharias.

\section{APPENDIX A}

Table 9 describes the contents of individual columns of the second data release catalog. The catalog is accessible online at www.rave-survey.org and via The Strasbourg astronomical Data Center (CDS) services.

\section{APPENDIX B EXTERNAL DATA}

Tables 10-14 compare the results of RAVE observations with those from the external datasets. The latter are discussed in Section 4.2.1.

\section{REFERENCES}

Anders, E., \& Grevesse, N. 1989, Geochimi. Cosmochim. Acta, 53, 197 Asplund, M., Grevesse, N., \& Sauval, J. 2006, Commun. Astroseismol., 147, 76 Famaey, B., Jorissen, A., Luri, X., Mayor, M., Udry, S., Dejonghe, H., \& Turon, C. 2005, A\&A, 430, 165
Fan, X., , Burstein, D., \& , Chen, J. S., , et al. 1996, AJ, 112, 628 Fagerholm, E. 1906, PhD Thesis, Uppsala Univ.

Fulbright, J. P., McWilliam, A., \& Rich, R. M. 2006, ApJ, 636, 821

Fulbright, J. P., McWilliam, A., \& Rich, R. M. 2007, ApJ, 661, 1152

Grevesse, N., \& Sauval, A. J. 1998, Space Sci. Rev., 85, 161

Gullberg, D., \& Lindegren, L. 2002, A\&A, 390, 383

Hambly, N. C., et al. 2001, MNRAS, 326, 1279

Høg, E., Fabricius, C., Makarov, V. V., Urban, S., Corbin, T., Wycoff, G., Bastian, U., Schwekendiek, P., \& Wicenec, A. 2000, A\&A, 355, L27

Keller, S. C., Schmidt, B. P., \& Bessell M. S., et al. 2007, Publ. Astron. Soc. Australia, 24,1

Latham, D. 2001, Radial Velocities, Encyclopedia of Astronomy and Astrophysics ed. P. Murdin (Bristol: IOP), article 1864

Lindegren, L. 1999, in ASP Conf. Ser. 185, Precise Stellar Radial Velocities, ed. J. B. Hearnshaw, \& C. D. Scarfe, (San Francisco, CA: ASP), 73

Montgomery, K. A., Marschall, L. A., \& Janes, K. A. 1993, AJ, 106, 181

Munari, U., Agnolin, P., \& Tomasella, A. 2001, BaltA, 10, 613

Munari, U., Sordo, R., Castelli, F., \& Zwitter, T. 2005a, A\&A, 442, 615

Munari, U., Fiorucci, M., \& the RAVE Collaboration, 2005, BAAS, 37, 1367

Nordström, B., Mayor, M., Andersen, J., Holmberg, J., Pont, F., Jorgensen, B. R., Olsen, E. H., Udry, S., \& Mowlavi, N. 2004, A\&A, 418, 989

Ocvirk, P., Pichon, C., Lançon, A., \& Thiébaut, E. 2006a, MNRAS, 365, 46

Ocvirk, P., Pichon, C., Lançon, A., \& Thiébaut, E. 2006b, MNRAS, 365, 74

Pichon, C., Siebert, A., \& Bienaymé, O. 2002, MNRAS, 329, 181

Randich, S., Sestito, P., Primas, F., Pallavicini, R., \& Pasquini, L. 2006, A\&A, 450,557

Rickman, H. 2001, Trans. of the IAU Proc. of the 24th General Assembly (San Francisco, CA: ASP), 91

Robin, A. C., Reylé, C., Derrière, S., \& Picaud, S. 2003, A\&A, 409, 523

Salaris, M., Chieffi, A., \& Straniero, O. 1993, ApJ, 414, 580

Seabroke, G. M., et al. 2008, MNRAS, 384, 11

Smith, M. C., et al. 2007, MNRAS, 379, 755

Soubiran, C., \& Girard, P. 2005, A\&A, 438, 139

Steinmetz, M. 2003, ASP Conf. Ser., 298, 381

Steinmetz, M., et al. 2006, AJ, 132, 1645 (Paper I)

Veltz, L., et al. 2008, A\&A, 480, 753

Zacharias, N., Urban, S. E., Zacharias, M. I., Wycoff, G. L., Hall, D. M., Germain, M. E., Holdenried, E. R., \& Winter, L. 2004, AJ, 127, 3043

Zwitter, T. 2002, A\&A, 386, 748

Zwitter, T., Castelli, F., \& Munari, U. 2004, A\&A, 417, 1055 